Dinâmica assintótica de um sistema de placas termoelásticas do tipo hiperbólico 

SERVIÇO DE PÓS-GRADUAÇÃO DO ICMC-USP

Data de Depósito:

Assinatura:

\title{
Dinâmica assintótica de um sistema de placas termoelásticas do tipo hiperbólico ${ }^{1}$
}

\author{
Alisson Rafael Aguiar Barbosa
}

\author{
Orientador: Prof. Dr. Ma To Fu
}

Tese apresentada ao Instituto de Ciências Matemáticas e de Computação - ICMC-USP, como parte dos requisitos para obtenção do título de Doutor em Ciências - Matemática. . VERSÃO REVISADA.

USP - São Carlos

Setembro de 2013

\footnotetext{
${ }^{1}$ Este trabalho teve apoio financeiro da CAPES sob o processo DS-5565094/D de 03/2009 à 10/2012
} 
Ficha catalográfica elaborada pela Biblioteca Prof. Achille Bassi e Seção Técnica de Informática, ICMC/USP, com os dados fornecidos pelo(a) autor(a)

\begin{tabular}{|c|c|}
\hline \multirow[t]{3}{*}{ B238d } & $\begin{array}{l}\text { Barbosa, Alisson Rafael Aguiar } \\
\quad \text { Dinâmica assintótica de um sistema de placas } \\
\text { termoelásticas do tipo hipebólico / Alisson Rafael } \\
\text { Aguiar Barbosa; orientador Ma To Fu. -- São Carlos, } \\
2013 \text {. } \\
\quad 74 \mathrm{p} \text {. }\end{array}$ \\
\hline & $\begin{array}{l}\text { Tese (Doutorado - Programa de Pós-Graduação em } \\
\text { Matemática)-- Instituto de Ciências Matemáticas e } \\
\text { de Computação, Universidade de São Paulo, } 2013 \text {. }\end{array}$ \\
\hline & $\begin{array}{l}\text { 1. Equações diferenciais parciais . } 2 \text {. } \\
\text { Termoelasticidade hiperbólica. 3. Atratores globais. } \\
\text { 4. Atratores exponenciais. I. Fu, Ma To, orient. } \\
\text { II. Título. }\end{array}$ \\
\hline
\end{tabular}




\section{Agradecimentos}

Agradeço primeiramente a minha esposa Carolina, que sempre me apoiou e esteve presente em muitos momentos importantes da minha vida. Agradeço também, de forma especial, a minha família.

Meus agradecimentos sinceros ao professor Dr. Ma To Fu, que não somente orientou este trabalho, mas se mostrou um grande profissional e uma pessoa de boa índole.

Aos professores do Programa de Doutorado do Instituto de Ciências Matemáticas e de Computação de São Carlos, com os quais tive a oportunidade de convívio.

Também não poderia esquecer dos amigos que fiz aqui ao longo desses quatro anos de curso, pois foram muitos os momentos felizes que compartilhamos juntos.

Finalmente, agradeço à CAPES pelo auxílio financeiro durante o período de 03/2009 e $10 / 2012$. 



\section{Resumo}

Este trabalho é dedicado ao estudo do comportamento a longo prazo de uma equação de placas estensíveis acoplada a uma equação de calor do tipo hiperbólico.

O problema corresponde a um modelo de termo-elasticidade baseado em teorias de calor do tipo não-Fourier. Considerando que efeitos de inércia de rotação estão presentes no modelo, mostramos que o efeito dissipativo do calor é suficiente para estabilizar exponencialmente o sistema, sem dissipações adicionais. Além disso, provamos que o sistema possui um atrator global de dimensão fractal finita e também atratores exponenciais. Nossos resultados generalizam e complementam diversos trabalhos existentes.

Palavras-chave: Equações diferenciais parciais, termoelasticidade, placas estensíveis, calor do tipo não-Fourier, atrator global, atratores exponenciais. 



\section{Abstract}

This work is concerned with long-time dynamics of solutions of extensible plate equations with thermal memory. It corresponds to a model of thermoelasticity based on a theory of non-Fourier heat flux. By considering the case where rotational inertia is present we show that the thermal dissipation is sufficient to stabilize the system exponentially and guarantee the existence of a finite-dimensional global attractor. In addition the existence of an exponential attractor and some further properties are also considered. Our results complements several existing results.

Key-words: Partial differential equations, thermoelasticity, extensible plates, thermal memory, non-Fourier heat flux, global attractor, exponential attractors. 


\section{Notações}

\section{Geral}

$|\Omega|:=$ medida de Lebesgue de $\Omega \subset \mathbb{R}^{N} ;$

$|\alpha|=\alpha_{1}+\alpha_{2}+\cdots+\alpha_{N} \quad$ e $\quad \alpha !=\alpha_{1} ! \alpha_{2} ! \cdots \alpha_{N} ! \quad$ para todo $\quad \alpha=$ $\left(\alpha_{1}, \ldots, \alpha_{N}\right) \in \mathbb{N}^{N}$

$p^{\prime}=\frac{p}{p-1}$ expoente conjugado de $p ;$

$x^{\alpha}=x_{1}^{\alpha_{1}} x_{2}^{\alpha_{2}} \cdots x_{N}^{\alpha_{N}} \quad$ para todo $\quad x=\left(x_{1}, \ldots, x_{N}\right) \in \mathbb{R}^{N} ;$

$\operatorname{supp}(u)=\overline{\{x \in \Omega ; u(x) \neq 0\}}^{\Omega} ;$

$\hookrightarrow$ inclusão contínua;

$\hookrightarrow \hookrightarrow$ inclusão compacta;

$\langle\cdot, \cdot\rangle$ dualidade;

$\left(X,\|\cdot\|_{X}\right)$ espaço de Banach.

\section{Operadores}

$$
\begin{aligned}
& D^{\alpha} u=\left\{\begin{array}{ccc}
\frac{\partial^{|\alpha|} u}{\partial x_{1}^{\alpha_{1}} \partial x_{2}^{\alpha_{2}} \ldots \partial x_{N}^{\alpha_{N}}} & \text { se } & \alpha \neq(0, \ldots, 0) \\
u & \text { se } & \alpha=(0, \ldots, 0)
\end{array}\right. \\
& \Delta u=\sum_{j=1}^{N} \frac{\partial^{2} u}{\partial x_{j}^{2}} \\
& \Delta^{2} u=\Delta(\Delta u)
\end{aligned}
$$


$\Delta_{p} u=\operatorname{div}\left(|\nabla u|^{p-2} \nabla u\right)$

$\mathcal{L}$-gerador infinitesimal de um $C^{0}$-semigrupo;

$e^{\mathcal{L}}$-semigrupo gerado pelo operador $\mathcal{L}$.

\section{Espaços de funções}

$$
\begin{aligned}
& C(\Omega)=\{u: \Omega \rightarrow \mathbb{R} \mid u \text { é contínua }\} \\
& C^{k}(\Omega)=\{u: \Omega \rightarrow \mathbb{R} \mid u \text { é } k \text {-vezes continuamente diferenciável }\} ; \\
& C^{\infty}(\Omega)=\{u: \Omega \rightarrow \mathbb{R} \mid u \text { é infinitamente diferenciável }\} ; \\
& C_{0}^{k}(\Omega)=\left\{u \in C^{k}(\Omega) \mid \operatorname{supp}(u) \subset \Omega \text { é compacto }\right\}, \quad k \in \mathbb{N} \text { ou } k=\infty ; \\
& C^{k, \alpha}(\Omega)=\left\{u \in C^{k}(\Omega) \mid D^{k} u \text { é } \alpha \text {-Hölder contínua }\right\} \\
& \mathcal{D}(\Omega):=\text { espaço das funções teste; } \\
& L^{p}(\Omega)=\left\{u: \Omega \rightarrow \mathbb{R} \mid u \text { é mensurável e } \int_{\Omega}|u(x)|^{p} d x<\infty\right\} ; \\
& L^{\infty}(\Omega)=\{u: \Omega \rightarrow \mathbb{R} \mid u \text { é mensurável e }|u(x)| \leq K \text { q.s. em } \Omega\} ; \\
& W^{m, p}(\Omega)=\left\{u \in L^{p}(\Omega)\left|D^{\alpha} u \in L^{p}(\Omega), \quad 0 \leq\right| \alpha \mid \leq m\right\} \\
& W_{0}^{m, p}(\Omega)={\overline{C_{0}^{\infty}(\Omega)}}^{W^{m, p}(\Omega)} \\
& H^{m}(\Omega)=W^{m, 2}(\Omega) \\
& H_{0}^{m}(\Omega)=W_{0}^{m, 2}(\Omega) \\
& L_{\mu}^{2}\left(\mathbb{R}^{+} ; X\right)=\left\{\eta: \mathbb{R}^{+} \rightarrow X \mid \int_{0}^{\infty} \mu(s)\|\eta(s)\|_{X}^{2} d s<\infty\right\} ; \\
& L^{p}(0, T ; X)=\left\{u:(0, T) \rightarrow X \mid u \text { é mensurável e } \int_{0}^{T}\|u(t)\|_{X}^{p} d t<\infty\right\} \\
& L^{\infty}(0, T ; X)= \\
& \left\{u:(0, T) \rightarrow X \mid u \text { é mensurável e }\|u(t)\|_{X} \leq K \text { q.s. em }(0, T)\right\} \text {; } \\
& C([0, T], X)=\{u:[0, T] \rightarrow X \mid u \text { é contínua de }[0, T] \text { em } X\} ;
\end{aligned}
$$




$$
\begin{aligned}
& C^{k}([0, T], X)= \\
& \{u:[0, T] \rightarrow X \mid u \text { é } k \text {-vezes continuamente diferenciável de }[0, T] \text { em } X\} ; \\
& C_{w}([0, T], X)=\{u:[0, T] \rightarrow X \mid u \text { é fracamente contínua de }[0, T] \text { em } X\} ; \\
& \mathcal{L}(X, Y)=\{T: X \rightarrow Y \mid T \text { é linear e contínua }\} ; \\
& \mathcal{C}(X)=\{T: X \rightarrow X \mid T \text { é contínua }\} .
\end{aligned}
$$

\section{Espaços duais}

$$
\begin{aligned}
& X^{\prime}=\mathcal{L}(X, \mathbb{R}) \text { dual de } X ; \\
& {\left[L^{p}(\Omega)\right]^{\prime}=L^{p^{\prime}}(\Omega), \quad 1 \leq p<\infty ;} \\
& {\left[W_{0}^{m, p}(\Omega)\right]^{\prime}=W^{-m, p^{\prime}}(\Omega), \quad 1 \leq p<\infty, m \in \mathbb{N} ;} \\
& {\left[H_{0}^{m}(\Omega)\right]^{\prime}=H^{-m}(\Omega), \quad m \in \mathbb{N} ;} \\
& {\left[L^{p}(0, T, X)\right]^{\prime}=L^{p^{\prime}}\left(0, T, X^{\prime}\right), \quad 1 \leq p<\infty ;} \\
& \mathcal{D}^{\prime}(\Omega)=\mathcal{L}(\mathcal{D}(\Omega), \mathbb{R}) ; \\
& \mathcal{D}^{\prime}(0, T ; X)=\mathcal{L}(\mathcal{D}(0, T), X) .
\end{aligned}
$$

\section{Convergências}

$\rightarrow$ convergência forte;

$\rightarrow$ convergência fraca;

$\stackrel{*}{*}$ convergência fraca estrela.

\section{Memória}

$$
(g * u)(t)=\int_{0}^{t} g(t-s) u(x, s) d s
$$

\section{Normas}

$$
\|u\|_{p}=\left(\int_{\Omega}|u(x)|^{p} d x\right)^{1 / p}, \quad \quad\|u\|_{\infty}=\sup _{x \in \Omega} \operatorname{ess}|u(x)|
$$




$$
\begin{aligned}
& \|u\|_{m, p}=\left(\sum_{|\alpha| \leq m}\left\|D^{\alpha} u\right\|_{p}^{p}\right)^{1 / p}, \quad\|u\|_{m, \infty}=\max _{|\alpha| \leq m}\left\|D^{\alpha} u\right\|_{\infty} ; \\
& \|u\|_{W_{0}^{1, p}}=\|\nabla u\|_{p} ; \\
& \|\eta\|_{\mu, X}=\left(\int_{0}^{\infty} \mu(s)\|\eta(s)\|_{X}^{2} d s\right)^{1 / 2} ; \\
& \|u\|_{L^{p}(0, T ; X)}=\left(\int_{0}^{T}\|u(t)\|_{X}^{p} d t\right)^{1 / p}, \quad\|u\|_{L^{\infty}(0, T ; X)}=\quad \sup _{t \in(0, T)} \operatorname{ess}\|u(t)\|_{X} ; \\
& \|u\|_{C^{k}([0, T], X)}=\sum_{j=0}^{k} \max _{t \in[0, T]}\left\|\frac{d^{j} u(t)}{d t^{j}}\right\| \|_{X} ; \\
& \|\varphi\|_{X^{\prime}}=\sup _{x \in X,\|x\|_{X} \leq 1}|\langle\varphi, x\rangle| ; \\
& \|T\|=\sup _{x \in X,\|x\|_{X}=1}\|T x\|_{Y}=\sup _{x \in X, x \neq 0} \frac{\|T x\|_{Y}}{\|x\|_{X}} .
\end{aligned}
$$





\section{Sumário}

1 Introdução $\quad 3$

2 Resultados básicos $\quad 8$

2.1 Espaços de Banach de Hilbert . . . . . . . . . . . . . . . . 8

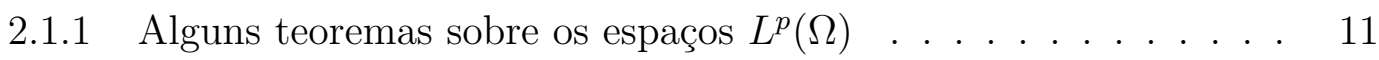

2.1.2 Teoremas de compacidade . . . . . . . . . . . . 13

2.1.3 Teoremas de análise funcional e imersão de Sobolev . . . . . . . 14

2.2 Operadores lineares não limitados . . . . . . . . . . . . . . . . . 15

2.2.1 Operador associado a uma forma bilinear . . . . . . . . 15

2.2.2 Potências fracionárias . . . . . . . . . . . . . . . 16

2.3 Semigrupos e atratores globais . . . . . . . . . . . . . . . . . . . 18

2.3.1 Semigrupos de operadores lineares definidos no espaço de Banach X 18

2.3.2 Problema de Cauchy abstrato . . . . . . . . . . . 20

2.3.3 Atratores globais e dimensão fractal finita . . . . . . . . . . . . 23

2.3.4 Sistemas dinâmicos quase-estáveis . . . . . . . . . . 26

2.3.5 Sistemas gradientes . . . . . . . . . . . . . 27

3 Boa colocação no sentido de Hadamard 30

3.1 Hipóteses e notações . . . . . . . . . . . . . . . . . . . . . . . . . . . . 32

3.2 Existência, unicidade e dependência contínua . . . . . . . . . . . . 34

3.2 .1 Existência local de soluções . . . . . . . . . . . . . . . 37

3.2.2 Prova do teorema principal . . . . . . . . . . . . . . 42 
4 Atratores de dimensão finita $\quad 49$

4.1 Sistema dinâmico gradiente . . . . . . . . . . . . . . 50

4.1.1 Conjunto das soluções estacionárias . . . . . . . . . . . . . 50

4.2 Sistema quase-estável . . . . . . . . . . . . . . . . . . . 53

4.2.1 Desigualdade de estabilização . . . . . . . . . . . . . 54

4.2 .2 Quase-estabilidade .................. 63

4.3 Demonstração do teorema $4.1 \ldots$. . . . . . . . . . . . . . . . . 64

5 Atratores exponenciais $\quad 66$

5.1 Atratores exponenciais generalizados . . . . . . . . . . 66

$\begin{array}{ll}\text { Referências Bibliográficas } & 71\end{array}$ 


\section{Capítulo 1}

\section{Introdução}

A presente tese se insere no estudo da dinâmica assintótica de sistemas termoelásticos não lineares. Mais precisamente, nossos resultados são dedicados ao estudo de placas elásticas extensíveis sob efeito dissipativo térmico originado por uma equação de calor hiperbólico, não satisfazendo, portanto, a lei de Fourier. Tais modelos termoelásticos são estudados como sistemas de equações diferenciais parciais do tipo

$$
\begin{aligned}
& u_{t t}+\Delta^{2} u-M\left(\int_{\Omega}|\nabla u|^{2}\right) \Delta u-c \Delta u_{t t}+f(u)+\nu \Delta \theta=h(x) \text { in } \Omega \times \mathbb{R}^{+}, \\
& \theta_{t}-\int_{0}^{\infty} k(s) \Delta \theta(t-s) d s-\nu \Delta u_{t}=0 \text { in } \Omega \times \mathbb{R}^{+},
\end{aligned}
$$

complementados com condições de fronteira e dados iniciais. Aqui $\Omega$ é uma região do plano que representa a placa em seu estado de repouso, $u$ representa deslocamentos verticais e $\theta$ a temperatura relativa ao meio ambiente. O núcleo $k$ da convolução é tomado como uma função convexa decrescente. Consideramos que todas as constantes físicas da teoria da elasticidade foram normalizadas. Nas aplicações em engenharia, a função $M$ é em geral uma reta crescente. O termo $M\left(\int_{\Omega}|\nabla u|^{2}\right) \Delta u$ contabiliza o efeito da extensibilidade da placa durante as vibrações transversais. Além disso, $f$ e $h$ representam forças do meio (fundação) e forças externas.

\section{O modelo de placas extensíveis}

No modelo clássico de placas de Euler-Bernoulli, as vibrações produzem apenas deslocamentos transversais. Entretanto, observamos que deformações tranversais produzem efeitos de extensibilidade da superfície da placa. Nesse sentido, 
Berger [4] e Woinowsky-Krieger [40] propuseram um modelo de placas (ou vigas) extensíveis. Em dimensão 1, o modelo se escreve como

$$
\frac{\partial^{2} u}{\partial t^{2}}+\frac{E I}{\rho} \frac{\partial^{4} u}{\partial x^{4}}-\left(\frac{H}{\rho}+\frac{E A}{2 \rho L} \int_{0}^{L}\left|\frac{\partial u}{\partial x}\right|^{2} d x\right) \frac{\partial^{2} u}{\partial x^{2}}=0
$$

em que $L, E, I, \rho, H$ e $A$ denotam, respectivamente, o comprimento da viga na posição de repouso, o módulo Young, o momento transversal de inércia, a densidade de massa, a tensão na posição de repouso e área de corte transversal. Queremos salientar que esse modelo de placas é um tema de pesquisa atual e relevante, conforme resultados recentes de Cavalcanti et al [9], Giorgi et al [21, 20], Ma et al [32] e Yang [44].

\section{A equação do calor do tipo hiperbólico}

$\mathrm{Na}$ formulação da equação do calor, a temperatura $\theta$ (em relação a um referencial) e o vetor fluxo de calor $q$ satisfazem a equação

$$
\theta_{t}+\beta \nabla \cdot q=0
$$

atribuída a Newton. Pela lei de Fourier, o fluxo de calor é proporcional ao gradiente da temperatura, isto é,

$$
q=-c \nabla \theta
$$

Portanto, eliminando-se $q$ das duas equações acima, obtemos a clássica equação do calor da teoria de Fourier

$$
\theta_{t}-c \beta \Delta \theta=0
$$

que é uma equação diferencial parabólica. Modelos matemáticos em termoelasticidade fundamentados na lei de condução de calor de Fourier produzem o "paradoxo" da velocidade de propagação infinita da energia térmica. Em outras palavras, ao aquecer um ponto de uma barra condutora de calor, o efeito é imediatamente sentido em todos os pontos da barra. Esse efeito regularizante se deve à natureza parabólica do modelo de Fourier. Modelos com velocidade infinita são menos realistas em aplicações envolvendo nanotecnologia ou micro-circuitos eletrônicos. Nesse caso, a engenharia tem utilizado alguns modelos de condução de calor hiperbólicos, fundamentados na lei de 
Cattaneo [8] ou também na lei de Gurtin e Pipkin [18]. Neste último caso, o fluxo de calor é definido como

$$
q(t)=-\int_{0}^{t} k(t-s) \nabla \theta(s) d s .
$$

Combinado com a lei de Newton (1.3), obtemos a equação do calor de Gurtin-Pipkin

$$
\theta_{t}-\beta \int_{0}^{t} k(t-s) \Delta \theta(s) d s,
$$

que é do tipo hiperbólica. A prova dessa afirmação é técnica e será apresentada no final dessa Introdução. Nossa pequena apresentação sobre condução de calor do tipo hiperbólico pode ser encontrada numa forma mais geral e completa no recente livro de Straughan [38].

\section{Revisão Bibliográfica e Resultados}

Discutimos agora alguns resultados da literatura que motivaram nosso trabalho. Veremos que o termo de inércia rotacional $-\Delta u_{t t}$ desempenha um papel importante.

Sobre a estabilidade assintótica (decaimento da energia a zero), Grasselli, Muñoz Rivera \& Pata [23] provaram que, na ausência do termo de inércia de rotação $-c \Delta u_{t t}$, o sistema linear com $c=M=f=h=0$ em (1.2) não é exponencialmente estável para uma grande classe de núcleos $k$.

Nesse contexto com $c=0$, em relação à existência de atratores, Wu [41] provou que o sistema (1.1)-(1.2) com $M=0$, mas $f(u)$ não linear, possui um atrator global. Entretanto, foi necessário adicionar uma dissipação forte $-\Delta u_{t}$ na equação da placa. Um problema mais geral no qual a equação linear de placas é substituída pelo modelo não linear extensível, isto é com $M \neq 0$, foi estudado por Potomkin [36]. Ele provou que o problema ( $\operatorname{com} c=0$ ) possui um atrator global e exponencial se adicionarmos uma dissipação fraca do tipo viscoelástico na equação da placa.

Por outro lado, Grasselli e Squassina [24] provaram que, na presença do termo de inércia de rotação, (isto é $c \neq 0$ ), a estabilidade exponencial do sistema linear associado a (1.2) é restaurada. 
Nesse contexto, na presença de inércia de rotação, a questão da existência de atratores não foi ainda considerada. Nossa contribuição segue, portanto, dessas observações.

Nosso problema é colocado da seguinte forma: estudamos o sistema (1.1)(1.2) na presença de inércia de rotação e considerando os efeitos da extensibilidade da placa $(M \neq 0)$. Nosso primeiro resultado principal (Teorema 3.1 do Capítulo 3) prova, utilizando a teoria de semigrupos, que o sistema (1.1)-(1.2), com condições de fronteira do tipo $u=\Delta u=0$, é bem colocado no sentido de Hadamard. Nosso segundo resultado principal (Teorema 4.1 do Capítulo 4) mostra que o sistema (1.1)-(1.2) possui um atrator global de dimensão fractal finita e também um atrator exponencial. Além disso, o atrator global é caracterizado como a variedade instável dos pontos de equilíbrio. Enfatizamos que nossos resultados são obtidos com a dissipação proveniente da equação do calor hiperbólico (equação em $\theta$ ). Não adicionamos nenhuma dissipação extra na equação da placa (equação em $u$ ). Esses resultados foram divulgados na referência Barbosa e Ma [3].

Sobre a metodologia, observamos que o nosso sistema é proveniente de equações diferenciais hiperbólicas e, portanto, não podemos lançar mão da regularidade parabólica para aplicar certos resultados abstratos da teoria dos sistemas dinâmicos de dimensão infinita. Nesse caso, existem técnicas que consideram a decomposição do semigrupo (operador solução) em uma parte uniformemente estável e outra parte compacta (cf. [6, 33]). Esse tipo de procedimento se revelou bastante complexo e técnico para o nosso problema. Como alternativa, seguimos de perto a metodologia apresentada por Chueshov e Lasiecka $[12,11]$ que é baseada no conceito de quasi-estabilidade (ver Definição 2.17).

\section{Apêndice: A equação de Gurtin-Pipkin é hiperbólica}

A lei de condução de calor de Cattaneo relaciona o fluxo de calor e o gradiente da temperatura com um atraso de tempo $\tau>0$. Assim,

$$
q(x, t+\tau)=-c \nabla \theta(x, t), \quad(\tau=\text { time-lag })
$$


Fazendo aproximação de Taylor de primeira ordem, obtemos

$$
\tau q_{t}+q=-c \nabla \theta
$$

Combinando essa equação com a de Newton (1.3), obtemos a nova equação do calor

$$
\tau \theta_{t t}+\theta_{t}-\beta c \Delta \theta=0
$$

que é claramente uma equação diferencial hiperbólica (equação de onda), com velocidade finita na propagação do calor.

Notemos que, se resolvermos a equação de Cattaneo [7] em relação a $q$, obtemos a equação de convolução

$$
q(t)=q(0) e^{-t / \tau}-\frac{c}{\tau} \int_{0}^{t} e^{-(t-s) / \tau} \nabla \theta(s) d s
$$

Fazendo $q(0)=0$ e $k(t-s)=\frac{c}{\tau} e^{-(t-s) / \tau}$, obtemos a equação de fluxo de GurtinPipkin (1.5). Assim, as equações de Gurtin-Pipkin e de Cattaneo são hiperbólicas. Ao leitor interessado, indicamos o recente trabalho de Fernández Sare \& Racke [15] que compara as teorias de Fourier e de Cattaneo na questão da estabilidade assintótica para um modelo termoelástico de vigas de Timoshenko. 



\section{Capítulo 2}

\section{Resultados básicos}

Apresentaremos neste capítulo algumas definições e resultados da Análise Funcional, tais como Espaços de Sobolev e Semigrupos. Como nosso objetivo é estabelecer um aparato teórico para nosso trabalho, não nos preocuparemos com provas formais para os teoremas que serão apresentados nesta etapa.

\subsection{Espaços de Banach de Hilbert}

As definições e resultados citados abaixo podem ser encontrados em Adams [1], Brezis [5], Kesavan [27], Kreyszig [28], Folland [16] e Lions [30]. Inicialmente, definiremos os espaços $L^{p}(\Omega)$. Como sabemos que os elementos são classes de equivalência de funções, por simplicidade vamos escrever funções ao invés de classe de equivalência.

Seja $\Omega \subset \mathbb{R}^{n}$ um aberto. Denotamos por $L^{p}(\Omega), 1 \leq p<\infty$, o conjunto das funções Lebesgue mensuráveis $u$, tal que $|u|^{p}$ é uma função integrável sobre $\Omega$. Isto é,

$$
L^{p}(\Omega)=\left\{u: \Omega \rightarrow \mathbb{R} \mid u \text { mensurável, } \int_{\Omega}|u(x)|^{p} d x<\infty\right\}
$$

munido com a norma usual

$$
\|u\|_{p}=\left(\int_{\Omega}|u(x)|^{p} d x\right)^{1 / p}, \quad u \in L^{p}(\Omega), \quad 1 \leq p<\infty
$$

Para $p=\infty$, definimos $L^{\infty}(\Omega)$ como sendo o conjunto das funções mensuráveis limita- 
das quase sempre (q.s) em $\Omega$, ou seja,

$$
L^{\infty}(\Omega)=\{u: \Omega \rightarrow \mathbb{R} \mid u \text { mensurável, }|u(x)| \leq K \text { q.s. em } \Omega\}
$$

munido com a norma

$$
\|u\|_{\infty}=\sup _{x \in \Omega} \operatorname{ess}|u(x)|=\inf A, \quad u \in L^{\infty}(\Omega),
$$

em que $A=\left\{K \in \mathbb{R}^{+}|| u(x) \mid \leq K\right.$ q.s. em $\left.\Omega\right\}$. Assim, $\left(L^{p}(\Omega),\|\cdot\|_{p}\right)$ é um espaço de Banach para $1 \leq p \leq \infty$. Além disso, $\left(L^{2}(\Omega)\right.$, \|\|$\left._{2}\right)$ é um espaço de Hilbert com $\|u\|_{2}=\sqrt{(u, u)}$, em que $(u, v)=\int_{\Omega} u(x) v(x) d x$ é o produto interno usual de $L^{2}(\Omega)$.

Dado um multi-índice $\alpha=\left(\alpha_{1}, \ldots, \alpha_{n}\right) \in \mathbb{N}^{n}$ e um ponto $x=\left(x_{1}, \ldots, x_{n}\right) \in \mathbb{R}^{n}$, definimos

$$
|\alpha|=\alpha_{1}+\alpha_{2}+\cdots+\alpha_{N}, \quad x^{\alpha}=x_{1}^{\alpha_{1}} x_{2}^{\alpha_{2}} \cdots x_{n}^{\alpha_{n}} \quad \text { e } \quad \alpha !=\alpha_{1} ! \alpha_{2} ! \cdots \alpha_{n} !
$$

O operador derivação de ordem $\alpha$ é definido como

$$
D^{\alpha} u=\left\{\begin{array}{ccc}
\frac{\partial^{|\alpha|} u}{\partial x_{1}^{\alpha_{1}} \partial x_{2}^{\alpha_{2}} \ldots \partial x_{n}^{\alpha_{n}}} & \text { se } & \alpha \neq(0, \ldots, 0), \\
u & \text { se } & \alpha=(0, \ldots, 0) .
\end{array}\right.
$$

Quando o multi-índice é da forma $\alpha=(0, \ldots, 0, i, 0, \ldots, 0) \in \mathbb{N}^{n}$, o operador derivação $D^{\alpha}$ também pode ser representado pelas seguintes notações

$$
D^{i}=D_{i}=\partial_{x_{i}}=\frac{\partial}{\partial x_{i}}=(\cdot)_{x_{i}}, \quad i=1, \ldots, n .
$$

Para $k=0,1,2, \ldots$ e um aberto $\Omega \subset \mathbb{R}^{n}$, definimos por $C^{k}(\Omega)$ o conjunto das funções $k$-vezes diferenciáveis sobre $\Omega$. Quando $k=0, C^{0}(\Omega)=C(\Omega)$ é o conjunto das funções contínuas sobre $\Omega$. Se $k=\infty, C^{\infty}(\Omega)$ é o conjunto das funções infinitamente diferenciáveis sobre $\Omega$. Denotamos também por $C_{0}^{\infty}(\Omega)$ as funções infinitamente diferenciáveis com suporte compacto sobre $\Omega$.

Finalmente, relembramos a definição dos espaços de Sobolev. Para $m \in \mathbb{N}$ e $1 \leq p \leq \infty$, denotamos por $W^{m, p}(\Omega)$ o conjunto das funções $u$ em $L^{p}(\Omega)$, de modo que suas derivadas $D^{\alpha} u, 0 \leq|\alpha| \leq m$, ainda pertençam a $L^{p}(\Omega)$, em que a derivada é no sentido das distribuições. Em termos de conjunto, temos

$$
W^{m, p}(\Omega)=\left\{u \in L^{p}(\Omega)\left|D^{\alpha} u \in L^{p}(\Omega), \quad 0 \leq\right| \alpha \mid \leq m\right\}
$$


munido com a norma

$$
\|u\|_{m, p}=\left(\sum_{0 \leq|\alpha| \leq m}\left\|D^{\alpha} u\right\|_{p}^{p}\right)^{1 / p}, \quad u \in W^{m, p}(\Omega) \quad 1 \leq p<\infty
$$

e

$$
\|u\|_{m, \infty}=\max _{0 \leq|\alpha| \leq m}\left\|D^{\alpha} u\right\|_{\infty}, u \in W^{m, \infty}(\Omega) .
$$

Assim, $\left(W^{m, p}(\Omega),\|\cdot\|_{m, p}\right)$ é um espaço de Banach. Além disso, definimos o conjunto $W_{0}^{m, p}(\Omega)$ como o subespaço de $W^{m, p}(\Omega)$ constituído pelo fecho de $C_{0}^{\infty}(\Omega)$ em $W^{m, p}(\Omega)$, ou seja,

$$
W_{0}^{m, p}(\Omega)={\overline{C_{0}^{\infty}(\Omega)}}^{W^{m, p}(\Omega)}
$$

Para o caso particular $p=2$, os espaços $W^{m, 2}(\Omega)=H^{m}(\Omega)$ e $W_{0}^{m, 2}(\Omega)=H_{0}^{m}(\Omega)$ são espaços de Hilbert com o produto interno usual dado por

$$
(f, g)_{H^{m}(\Omega)}=\sum_{|\alpha| \leq m}\left(D^{\alpha} f, D^{\alpha} g\right)_{L^{2}(\Omega)}, \quad f, g \in H^{m}(\Omega) .
$$

Naturalmente, denota-se $W^{0, p}(\Omega)=L^{p}(\Omega)$.

Sejam $X$ um espaço de Banach com norma $\|\cdot\|_{X}$ e $H$ um espaço de Hilbert com produto interno $(\cdot, \cdot)_{H}$ e norma $\|\cdot\|_{H}$. Para $1 \leq p<\infty$, representaremos por $L^{p}(0, T ; X)$ o conjunto de funções vetoriais mensuráveis $u:(0, T) \rightarrow X$, tal que $\|u(\cdot)\|_{X}$ pertence a $L^{p}(0, T)$. Mais precisamente,

$$
L^{p}(0, T ; X)=\left\{u:(0, T) \rightarrow X \mid u \text { mensurável, } \int_{0}^{T}\|u(t)\|_{X}^{p} d t<\infty\right\} .
$$

O espaço $L^{p}(0, T ; X)$ equipado com a norma

$$
\|u\|_{L^{p}(0, T ; X)}=\left(\int_{0}^{T}\|u(t)\|_{X}^{p} d t\right)^{1 / p}, \quad u \in L^{P}(0, T, X),
$$

é um espaço de Banach. Quando $p=\infty$, o espaço $L^{\infty}(0, T ; X)$ é o conjunto de funções vetoriais mensuráveis $u:(0, T) \longrightarrow X$, de forma que $\|u(\cdot)\|_{X}$ pertence a $L^{\infty}(0, T)$. Isto é,

$$
L^{\infty}(0, T ; X)=\left\{u:(0, T) \rightarrow X \mid u \text { mensurável, }\|u(t)\|_{X} \leq K \text { q.s. em }(0, T)\right\} .
$$


Em $L^{\infty}(0, T ; X)$, definimos a norma dada por

$$
\|u\|_{L^{\infty}(0, T ; X)}=\operatorname{supess}\left\{\|u(t)\|_{X} ; t \in(0, T)\right\}
$$

Com essa norma, $L^{\infty}(0, T ; X)$ é um espaço de Banach. Por fim, definiremos o espaço $L_{\mu}^{2}$. Dada uma função "peso"

$$
\mu \in C\left(\mathbb{R}^{+}\right) \cap L^{1}\left(\mathbb{R}^{+}\right),
$$

definimos o seguinte espaço de Hilbert

$$
L_{\mu}^{2}\left(\mathbb{R}^{+} ; H\right)=\left\{\xi: \mathbb{R}^{+} \rightarrow H \mid \int_{0}^{\infty} \mu(s)\|\xi(s)\|_{H}^{2} d s<\infty\right\}
$$

munido com produto interno

$$
\left(\xi_{1}, \xi_{2}\right)_{\mu, H}=\int_{0}^{\infty} \mu(s)\left(\xi_{1}(s), \xi_{2}(s)\right)_{H} d s, \quad \forall \xi_{1}, \xi_{2} \in L_{\mu}^{2}\left(\mathbb{R}^{+} ; H\right)
$$

e norma

$$
\|\xi\|_{\mu, H}^{2}=\int_{0}^{\infty} \mu(s)\|\xi(s)\|_{H}^{2} d s, \quad \forall \xi \in L_{\mu}^{2}\left(\mathbb{R}^{+} ; H\right)
$$

\subsubsection{Alguns teoremas sobre os espaços $L^{p}(\Omega)$}

Nesta subseção, abordaremos alguns teoremas sobre os espaços $L^{p}(\Omega)$, $1 \leq p \leq \infty$, em que $\Omega$ é um domínio, isto é, um conjunto aberto e mensurável do $\mathbb{R}^{n}$. Nosso propósito com essa apresentação é apenas dar uma referência para o leitor, sem entrar em detalhes. Para as demonstrações, sugerimos Adams [1], Brézis [5], Kreyszig [28], kesavan[27] e Simon [37].

Lema 2.1. Seja $\Omega$ um domínio de $\mathbb{R}^{N}$.

(i) Se $1<p<\infty$, então $L^{p}(\Omega)$ é reflexivo.

(ii) Se $1 \leq p<\infty$, então $L^{p}(\Omega)$ é separável.

\section{Observação 2.1.}

(i) $L^{1}(\Omega)$ e $L^{\infty}(\Omega)$ não são reflexivos. 
(ii) $L^{\infty}(\Omega)$ não é separável.

Teorema 2.1 (Teorema de Densidade). Seja $\Omega$ um dominio de $\mathbb{R}^{N}$.

(i) Se $k \geq 0$, então $C_{0}^{k}(\Omega)$ é denso em $L^{p}(\Omega)$, para $1 \leq p<\infty$.

(ii) Se $\Omega$ é de classe $C^{m}, m \geq 1$, então $C^{m}(\bar{\Omega})$ é denso em $W^{m, p}(\Omega)$, para $1 \leq p<\infty$.

Teorema 2.2 (Teorema de Interpolação). Seja $\Omega \subset \mathbb{R}^{n}$ um domínio limitado com fronteira suave. Suponhamos que

$$
\begin{array}{rrr}
p \leq q \leq \infty & \text { se } & m p>n, \\
p \leq q<\infty & \text { se } & m p=n, \\
p \leq q \leq \frac{N p}{N-m p} \quad \text { se } & m p<n .
\end{array}
$$

Então existe uma constante $K=K(n, m, p, q, \Omega)>0$ tal que, para toda $u \in W^{m, p}(\Omega)$,

$$
\|u\|_{q} \leq K\|u\|_{m, p}^{\theta}\|u\|_{p}^{1-\theta}
$$

em que $\theta=(n / m p)-(n / m q)$.

Teorema 2.3 (Desigualdade de Poincaré). Seja $\Omega \subset \mathbb{R}^{n}$ um domínio limitado e $1 \leq p<\infty$. Então existe uma constante $C=C(p,|\Omega|)>0$ tal que

$$
\|u\|_{p} \leq C\|\nabla u\|_{p}, \quad \forall u \in W_{0}^{1, p}(\Omega) .
$$

Teorema 2.4 (Fórmula de Green). Seja $\Omega \subset \mathbb{R}^{n}$ um aberto limitado com fronteira $\Gamma$ suave. Se $u, \in H^{2}(\Omega)$ e $v \in H^{1}(\Omega)$, então

$$
-\int_{\Omega}(\Delta u) v d x=\int_{\Omega} \nabla u \cdot \nabla v d x-\int_{\Gamma} \frac{\partial u}{\partial \nu} v d S
$$

em que $\nu$ representa o vetor normal unitário exterior a $\Gamma$ e $\frac{\partial u}{\partial \nu}:=\nabla u \cdot \nu$ a derivada normal de $u$.

Teorema 2.5 (Desigualdade de Hölder). Sejam $1<p, q<\infty \operatorname{com} \frac{1}{p}+\frac{1}{q}=1$ e $\Omega \subset \mathbb{R}^{n}$. Se $u \in L^{p}(\Omega)$ e $v \in L^{q}(\Omega)$, então $u v \in L^{1}(\Omega)$ e

$$
\int_{\Omega}|u(x) v(x)| d x \leq\|u\|_{p}\|v\|_{q}
$$

$A$ desigualdade também vale se $p=1$ e $q=\infty$. 
Lema 2.2 (Desigualdade de Gronwall). Sejam $\alpha \geq 0$ uma constante e $\phi \in L^{\infty}(a, b)$, $\beta \in L^{1}(a, b)$ tais que $\beta>0, \phi \geq 0$. Se

$$
\phi(t) \leq \alpha+\int_{a}^{b} \beta(s) \phi(s) d s, \quad a \leq t \leq b,
$$

então

$$
\phi(t) \leq \alpha e^{\int_{a}^{b} \beta(s) d s}, \quad a \leq t \leq b .
$$

Lema 2.3 (Desigualdade de Young). Seja $1<p, q<\infty \operatorname{com} \frac{1}{p}+\frac{1}{q}=1$. Então

$$
a b \leq \frac{a^{p}}{p}+\frac{b^{q}}{q}, \quad \forall a, b \geq 0 .
$$

Lema 2.4 (Desigualdade de Young $\operatorname{com} \epsilon$ ). Seja $1<p, q<\infty \operatorname{com} \frac{1}{p}+\frac{1}{q}=1$ e $\epsilon>0$ qualquer. Então

$$
a b \leq \epsilon a^{p}+C_{\epsilon} b^{q}, \quad \forall a, b \geq 0,
$$

em que $C_{\epsilon}=(\epsilon p)^{-q / p} q^{-1}$.

Lema 2.5. Seja $X$ um espaço de Banach. Se $f \in L^{p}(0, T ; X)$ e $\frac{d f}{d t} \in L^{p}(0, T ; X)$, então $f \in C([0, T], X)$, a menos de um conjunto de medida nula em $[0, T]$.

\subsubsection{Teoremas de compacidade}

Abaixo listaremos alguns resultados clássicos sobre compacidade. Como na seção anterior, não temos a pretensão de esboçar demonstrações ou discutir detalhes sobre os teoremas mencionados. Nossa intenção é apenas apresentar uma referência ao leitor. Detalhes podem ser encontrados em Lions [30] e Lions \& Magenes [31].

Teorema 2.6 (Aubin-Lions). Sejam $X_{0}, X, X_{1}$ três espaços de Banach com $X_{0}$ e $X_{1}$ reflexivos. Suponhamos que $X_{0} \hookrightarrow X \hookrightarrow X_{1}$, em que a primeira inclusão é compacta. Além disso, para quaisquer $p_{0}, p_{1}$ com $1<p_{0}, p_{1}<\infty$, consideremos o espaço

$$
W=\left\{u \mid u \in L^{p_{0}}\left(0, T ; X_{0}\right), u_{t} \in L^{p_{1}}\left(0, T ; X_{1}\right)\right\}
$$

munido da norma $\|u\|_{W}=\|u\|_{L^{p_{0}\left(0, T ; X_{0}\right)}}+\left\|u_{t}\right\|_{L^{p_{1}\left(0, T ; X_{1}\right)}}$. Então,

$$
W \hookrightarrow \hookrightarrow L^{p_{0}}(0, T ; X)
$$

em que $X_{0} \hookrightarrow \hookrightarrow X$ indica que $X_{0}$ tem imersão compacta em $X$. 
Teorema 2.7 (Compacidade Fraca). Seja X um espaço de Banach reflexivo. Se $B \subset X$ é limitado, então $B$ é compacto na topologia fraca $\sigma\left(X, X^{\prime}\right)$, isto é, qualquer sequência $\left\{x_{n}\right\} \subset B$ possui uma subsequência $\left\{x_{n_{k}}\right\}$ convergente em $X$ na topologia fraca $\sigma\left(X, X^{\prime}\right)$.

Teorema 2.8 (Compacidade Fraca Estrela). Seja X um espaço de Banach separável. Se $F \subset X^{\prime}$ é limitado, então $F$ é compacto na topologia fraca estrela $\sigma\left(X^{\prime}, X\right)$, isto é, qualquer sequência $\left\{f_{n}\right\} \subset F$ possui uma subsequência $\left\{f_{n_{k}}\right\}$ convergente em $X^{\prime}$ na topologia fraca estrela $\sigma\left(X^{\prime}, X\right)$.

\subsubsection{Teoremas de análise funcional e imersão de Sobolev}

Teorema 2.9 (Teorema de Representação de Riesz-Fréchet). Seja $(\cdot, \cdot): H \times H \rightarrow \mathbb{R}$ um produto interno em $H$. O par $\left(H,\|\cdot\|_{H},(\cdot, \cdot)_{H}\right)$ denotará o espaço de Hilbert munido com esse produto interno. Então, para todo funcional $\varphi \in H^{\prime}$, existe um único $f \in H$ tal que

$$
\langle\varphi, v\rangle_{H^{\prime}, H}=(f, v)_{H}, \quad \forall v \in H .
$$

Além disso, $\|\varphi\|_{H^{\prime}}=\|f\|_{H}$.

Teorema 2.10 (Teorema de Lax-Milgram). Seja $(\cdot, \cdot): H \times H \rightarrow \mathbb{R}$ um produto interno em H. Sejam $\left(H,\|\cdot\|_{H},(\cdot, \cdot)_{H}\right)$ o espaço de Hilbert minido com esse produto interno e $a(u, v)$ uma forma bilinear contínua e coerciva. Então para todo funcional $\varphi \in H^{\prime}$, existe um único $u \in H$ tal que

$$
a(u, v)=\langle\varphi, v\rangle_{H^{\prime}, H}, \quad \forall v \in H .
$$

Teorema 2.11. Seja $\Omega \subset \mathbb{R}^{n}$ um domínio limitado com fronteira de classe $C^{m}$.

i. Se $m p<n$, a seguinte inclusão contínua

$$
W^{m, p}(\Omega) \hookrightarrow L^{q^{*}}(\Omega), \quad \text { em que } \quad \frac{1}{q^{*}}=\frac{1}{p}-\frac{m}{n}
$$

é válida. Além disso, a inclusão é compacta para qualquer $q$, com $p \leq q<q^{*}$. 
ii. Se $m p=n$, então a seguinte inclusão é contínua e compacta

$$
W^{m, p}(\Omega) \hookrightarrow L^{q}(\Omega), \quad \text { para todo } \quad 1 \leq q<\infty
$$

Além disso, se $p=1$ e $m=n$, a mesma relação acima vale para $q=\infty$.

iii. Se $k+1>m-\frac{n}{p}>k, k \in \mathbb{N}$, então, escrevendo $m-\frac{n}{p}=k+\alpha$, com $0<\alpha<1$, temos a seguinte inclusão contínua

$$
W^{m, p}(\Omega) \hookrightarrow C^{k, \alpha}(\bar{\Omega})
$$

em que $C^{k, \alpha}(\bar{\Omega})$ representa o espaço das funções em $C^{k}(\bar{\Omega})$ cujas derivadas de ordem $k$ são $\alpha$-Hölder contínuas. Além disso, se $n=m-k-1, \alpha=1$ e $p=1$, a inclusão vale também para $\alpha=1$ e a inclusão $W^{m, p}(\Omega) \hookrightarrow C^{k, \beta}(\bar{\Omega})$ é compacta para todo $0 \leq \beta<\alpha$.

A demonstração desse Teorema pode ser encontrada em Adams [1].

\subsection{Operadores lineares não limitados}

Nesta seção, faremos um breve revisão de operadores lineares associados a uma forma bilinear. Para mais detalhes sobre o assunto, recomendamos os trabalhos de Temam [39], Yosida [42] e Zheng [43].

\subsubsection{Operador associado a uma forma bilinear}

$\operatorname{Sejam}\left(V,\|\cdot\|_{V},(\cdot, \cdot)_{V}\right)$ e $\left(H,\|\cdot\|_{H},(\cdot, \cdot)_{H}\right)$ dois espaços de Hilbert de modo que $V$ é denso em $H$, com inclusão $V \hookrightarrow H$ compacta. O espaço $V^{\prime}$ é dual de $V$ e denotamos por $\langle\cdot, \cdot\rangle$ a dualidade entre $V^{\prime}$ e $V$. Identificando $H$ com seu dual, por meio do Teorema da representação de Riesz, obtemos a seguinte cadeia de inclusões

$$
V \hookrightarrow H \cong H^{\prime} \hookrightarrow V^{\prime}
$$

Seja $a(\cdot, \cdot): V \times V \rightarrow \mathbb{R}$ uma forma bilinear e contínua. Definimos o operador linear $A: V \rightarrow V^{\prime}$ dado por

$$
\langle A u, v\rangle=a(u, v), \quad \forall u, v \in V
$$


com domínio

$$
D(A)=\{u \in V \mid A u \in H\} .
$$

Assim, o operador linear $A$ fica definido pela terna $\{V, H, a(\cdot, \cdot)\}$.

Sabemos da teoria de análise funcional (ver Temam [39, Capítulo 2]) que, se $a(\cdot, \cdot)$ for uma forma bilinear contínua, coerciva e simétrica, então o operador linear $A: D(A) \subset H \rightarrow H$ é fechado, não limitado, positivo definido, autoadjunto e uma bijeção (isomorfismo). Além disso, o domínio $D(A)$ com a norma $\|u\|_{D(A)}=\|A u\|_{H}$ (que é equivalente a norma do gráfico $\|u\|_{G}^{2}=\|u\|_{H}^{2}+\|A u\|_{H}^{2}$ ), é um espaço de Hilbert denso em $H$.

Observação 2.2. Um exemplo clássico de uma forma bilinear que satisfaz as condições acima é dada pelo produto interno $(\cdot, \cdot)_{V}$ em $V$. Nesse caso, considerando o operador $A$ dado pela terna $\left\{V, H,(\cdot, \cdot)_{V}\right\}$, temos $A: D(A) \subset V \rightarrow H$ de modo que

$$
(A u, v)_{H}=(u, v)_{V}, \quad \forall u \in D(A), \quad \forall v \in V .
$$

Mediante às condições satisfeitas pelo operador $A$, e considerando que a imersão $V \hookrightarrow H$ é compacta, segue da teoria espectral (ver por exemplo Yosida [42]) que existe uma base ortonormal completa $\left\{w_{j}\right\}_{j \in \mathbb{N}}$ de $H$ e uma sequência de números reais $\left\{\lambda_{j}\right\}_{j \in \mathbb{N}}$ tais que

$$
\begin{aligned}
& 0<\lambda_{1} \leq \lambda_{2} \leq \cdots \quad \text { com } \quad \lambda_{j} \rightarrow \infty \quad \text { quando } \quad j \rightarrow \infty \\
& w_{j} \in D(A) \quad \text { e } \quad A w_{j}=\lambda_{j} w_{j}, \quad \forall j \in \mathbb{N} .
\end{aligned}
$$

Observamos também que

$$
\begin{aligned}
& \left(w_{i}, w_{j}\right)_{H}=\delta_{i j} \quad \mathrm{e} \\
& a\left(w_{i}, w_{j}\right)=\lambda_{i} \delta_{i j}, \quad \forall i, j \in \mathbb{N},
\end{aligned}
$$

em que $\delta_{i j}$ é o delta de Kronecker.

\subsubsection{Potências fracionárias}

Se o operador $A$ satisfaz as propriedades da subseção anterior e possui a base dada em (2.1)-(2.2), podemos definir as potências fracionárias do operador $A$, ou 
seja, $A^{s}$, para $s \in \mathbb{R}$. Mais ainda, os operadores $A^{s}$ podem ser caracterizados em termos da base $\left\{w_{j}\right\}_{j \in \mathbb{N}}$.

Notamos inicialmente que, para $s>0$, o operador

$$
A^{s}: D\left(A^{s}\right) \subset H \rightarrow H
$$

é um operador linear não limitado, positivo definido, autoadjunto e injetivo, cujo domínio $D\left(A^{s}\right)$ é denso em $H$. Atribuindo a $D\left(A^{s}\right)$ o produto interno e norma

$$
(u, v)_{D\left(A^{s}\right)}=\left(A^{s} u, A^{s} v\right)_{H} \quad \text { e } \quad\|u\|_{D\left(A^{s}\right)}=\left\|A^{s} u\right\|_{H},
$$

concluímos que $D\left(A^{s}\right)$ é um espaço de Hilbert. Além disso, $D\left(A^{-s}\right)$ é definido como sendo o dual de $D\left(A^{s}\right)$. Portanto, o operador $A^{s}$ pode ser estendido como um isomorfismo de $H$ em $D\left(A^{-s}\right)$. Em $D\left(A^{-s}\right)$, consideramos o produto interno e norma como acima, substituindo $s$ por $-s$.

Utilizando novamente a imersão compacta $V \hookrightarrow H$, pode-se definir $A^{s}$ em termos da base $\left\{w_{j}\right\}_{j \in \mathbb{N}}$ por

$$
A^{s} u=\sum_{j=1}^{\infty} \lambda_{j}^{s}\left(u, w_{j}\right)_{H} w_{j}, \quad \forall u \in D\left(A^{s}\right),
$$

em que

$$
D\left(A^{s}\right)=\left\{\left.u \in V\left|\sum_{j=1}^{\infty} \lambda_{j}^{2 s}\right|\left(u, w_{j}\right)_{H}\right|^{2}<\infty\right\},
$$

para cada $s>0$. Nesse caso, a norma em $D\left(A^{s}\right)$ pode ser reescrita como

$$
\|u\|_{D\left(A^{s}\right)}=\left(\sum_{j=1}^{\infty} \lambda_{j}^{2 s}\left|\left(u, w_{j}\right)_{H}\right|^{2}\right)^{1 / 2}, \quad \forall u \in D\left(A^{s}\right) .
$$

Ademais, $D\left(A^{-s}\right)$ é o complemento de $H$ para a norma $\left(\sum_{j=1}^{\infty} \lambda_{j}^{2 s}\left|\left(u, w_{j}\right)_{H}\right|^{2}\right)^{1 / 2}$ e $A^{-s}$ é definido como acima com $-s$ no lugar de $s$. Teremos o seguinte resultado:

Lema 2.6. Se $\alpha, \beta \in \mathbb{R}$ então $D\left(A^{\beta}\right) \hookrightarrow \hookrightarrow D\left(A^{\alpha}\right)$, para $\beta>\alpha$.

Para detalhes, ver Grasselli, Rivera \& Patta [23]. 


\subsection{Semigrupos e atratores globais}

Nesta seção, abordaremos a definição e os teoremas que caracterizam o gerador infinitesimal de um semigrupo de classe $C_{0}$. Também apresentaremos a definição e os teoremas que estabelecem a existência do Atrator Global com dimensão finita e propriedades geométricas. Abordaremos, por fim, o conceito de Atrator Exponencial Generalizado. De agora em diante, $\left(X,\|\cdot\|_{X}\right)$ sempre será um espaço de Banach, $\left(H,\|\cdot\|_{H},(\cdot, \cdot)_{H}\right)$ um espaço de Hilbert e $(\mathcal{L}(X),\|\cdot\|)$ o espaço dos operadores lineares e contínuos em $X$.

\subsubsection{Semigrupos de operadores lineares definidos no espaço de Banach X}

Definição 2.1. Seja X um espaço de Banach. Uma família $\{S(t)\}_{t \geq 0}$ de operadores lineares e limitados é chamada de semigrupo de operadores lineares limitados, (ou simplesmente semigrupo), quando são verificadas as seguintes condições

i. $S(0)=I d: X \rightarrow X \quad$ (Operador Identidade em $X)$.

ii. $S(t+s)=S(t) S(s)$, para cada $t, s \geq 0$.

iii. Além disso, dizemos que $\{S(t)\}_{t \geq 0}$ é um semigrupo de classe $C_{0}$, (ou simplesmente $C_{0}$-semigrupo), se tivermos

$\lim _{t \rightarrow 0^{+}}\|[S(t)-I d] x\|_{X}=0$, para todo $x \in X$.

Definição 2.2. Um operador A é chamado de gerador infinitesimal de um semigrupo $\{S(t)\}_{t \geq 0}$ se A é definido como

$$
D(A)=\left\{x \in X \mid \lim _{t \rightarrow 0^{+}} \frac{S(t)-I d}{t} x \text { existe }\right\}
$$

e, para cada $x \in D(A)$, tivermos

$$
A x=\lim _{t \rightarrow 0^{+}} \frac{S(t)-I d}{t} x .
$$

Ás vezes, diz-se também que o semigrupo $S(t)$ é gerado pelo operador $A$. 
Definição 2.3. Um semigrupo $\{S(t)\}_{t \geq 0}$ é chamado de uniformemente limitado se existe uma constante $M \geq 1$ tal que

$$
\|S(t)\|_{X} \leq M, \quad \forall t \geq 0
$$

Quando $M \leq 1$, diremos também que $\{S(t)\}_{t \geq 0}$ é um semigrupo linear de contrações.

Definição 2.4. Seja A um operador linear definido sobre um espaço de Hilbert $H$ com domínio $D(A) \subseteq H$. Dizemos que A é um operador dissipativo quando

$$
R e(A x, x)_{H} \leq 0, \quad \forall x \in D(A)
$$

Seguem algumas observações sobre propriedades de semigrupos de classe $C_{0}$. A prova de tais propriedades pode ser encontrada em Pazy [35].

\section{Observação 2.3.}

1. Se $\{S(t)\}_{t \geq 0}$ é um semigrupo de classe $C_{0}$, então $\|S(t)\|_{X}$ é uma função limitada em qualquer intervalo limitado $[0, T]$.

2. Todo semigrupo de classe $C_{0}$ é fortemente contínuo em $\mathbb{R}^{+}$, ou seja, se $t \in \mathbb{R}^{+}$, então $\lim _{s \rightarrow t} S(s) x=S(t) x, \forall x \in X$.

3. Seja $\{S(t)\}_{t \geq 0}$ um semigrupo de classe $C_{0}$. Então existem constantes $w>0$ e $M \geq 1$ tal que

$$
\|S(t)\| \leq M e^{w t}, \quad \forall t \geq 0
$$

Quando $w<0$, é possivel escolher $w=0$ e uma constante $M \geq 1$ de modo que $\|S(t)\| \leq M$, para todo $t \geq 0$. Por isso, $\{S(t)\}_{t \geq 0}$ é um semigrupo uniformemente limitado de classe $C_{0}$. Caso $M \leq 1$, teremos que $\{S(t)\}_{t \geq 0}$ é semigrupo de contrações de classe $C_{0}$.

4. Seja A o gerador de um semigrupo de classe $C_{0},\{S(t)\}_{t \geq 0}$. Se $x \in D(A)$ e $t \geq 0$, então $S(t) x \in D(A)$ e a função de $\mathbb{R}^{+}$em $X$, dada por $t \mapsto S(t) x$, é diferenciável $e$

$$
\frac{d S(t) x}{d t}=A S(t) x=S(t) A x, \quad x \in D(A) .
$$


5. O gerador infinitesimal de um semigrupo de classe $C_{0}$ é um operador fechado com domínio denso em $X$.

Podemos caracterizar um gerador infinitesimal de um $C_{0}$-semigrupo através dos teoremas de Hille-Yosida e Lumer-Phillips. Apresentaremos aqui apenas o último teorema mencionado, que fornece uma caracterização para os $C_{0^{-}}$-semigrupos de contração suficiente para nosso trabalho. Para detalhes sobre os teoremas de Hille-Yosida e Lumer-Phillips, ver Pazy [35].

Definição 2.5. Seja A um operador linear definido em um espaço de Banach X. $O$ conjunto dos $\lambda \in \mathbb{C}$, tal que o operador linear $\lambda I-A$ é inversível, tem inverso limitado e domínio denso em $X$, é chamado conjunto resolvente de $A$ e é representado por $\rho(A)$. $O$ operador linear $R(\lambda, A)=(\lambda I-A)^{-1}$ é dito Resolvente de $A$.

Teorema 2.12 (Lumer-Phillips). Seja A um operador linear com dominio $D(A)$ denso em um espaço de Hilbert H. Temos:

1. Se $A$ é dissipativo e existe um número $\lambda>0$ tal que $\mathcal{I} m(\lambda I-A)=H$, então $A$ é gerador infinitesimal de um $C_{0}$-semigrupo de contrações em $H$.

2. Se A é o gerador infinitesimal de um $C_{0}$-semigrupo de contrações em $H$, então $\mathcal{I} m(\lambda I-A)=H$ para todo $\lambda>0$ e A é dissipativo.

\subsubsection{Problema de Cauchy abstrato}

Como é bem conhecido, a teoria geral de semigrupos nos fornece ferramentas para estudar o problemas de valor inicial abstrato

$$
\left\{\begin{array}{l}
\frac{d}{d t} U(t)=A U(t)+F(U(t)), \quad t>0, \\
U(0)=U_{0}
\end{array}\right.
$$

em que $A$ é um operador linear com domínio $D(A) \subset X, X$ um espaço de Banach e $F: X \rightarrow X$ é uma função. Enunciaremos alguns resultados que nos darão subsídios para estipularmos quando o problema abstrato (2.6) possui solução. Os resultados abaixo, são casos partículares dos teoremas apresentados em Pazy [35] Capítulo 6. 
Observação 2.4. Em geral, pedimos que $F:[0, \infty) \times X \rightarrow X$ seja uma função, contínua em $t \geq 0$, é localmente Lipschtz contínua em $U \in X$, uniformemente para $t$ em intervalos limitados, isto é, para qualquer $T>0$ e constante $c \geq 0$, existe uma constante $L=L(c, T)$ tal que

$$
\|F(t, U)-F(t, V)\| \leq L\|U-V\|
$$

é satisfeita para todo $U, V \in X$ com $\|U\| \leq c,\|V\| \leq c$ e $t \in[0, T]$. No nosso caso, como $F$ independe de $t \in[0, \infty)$, temos essas condições.

Seguem algumas definições sobre os tipos de soluções para o problema abstrato dado acima.

Definição 2.6. Uma solução clássica do problema de Cauchy (2.6) é uma função

$$
U:[0, T) \rightarrow X
$$

na qual $U(t)$ é contínua em $[0, T)$ continuamente diferenciável em $(0, T), U(t) \in D(A)$ para $t>0$ e satisfaz (2.6) em $[0, T)$.

Definição 2.7. Uma solução forte do problema de Cauchy (2.6) é uma função

$$
U:[0, T] \rightarrow X
$$

tal que $U(t)$ é contínua para $t \in[0, T]$, continuamente diferenciável quase sempre em $[0, T], u_{t} \in L^{1}(0, T ; X)$ e satisfaz (2.6) em $[0, T]$ quase sempre.

A próxima definição estabelece um tipo de solução fraca, chamada de mild solution, para o problema (2.6).

Definição 2.8. Sejam $X$ espaço de Banach e A gerador infinitesimal de um $C^{0}$ semigrupo, $U_{0} \in X$. Dizemos que uma função $U \in C([0, T] ; X)$ é uma solução fraca (Mild Solution) para o problema (2.6) se u satisfaz a seguinte equação integral

$$
U(t)=S(t) U_{0}+\int_{0}^{t} S(t-s) F(U(s)) d s, \quad t \in[0, T] .
$$


A partir de agora, quando mencionarmos "solução fraca", estaremos nos referindo a mild solution. Os próximos resultados fornecerão subsídios para que possamos dizer quando o problema abstrato (2.6) possui solução.

Teorema 2.13. Sejam $X$ espaço de Banach e $A$ gerador infinitesimal de um $C_{0^{-}}$ semigrupo. Assumimos que F é uma função contínua, localmente Lipschitz contínua em $U \in X$. Então, para todo $x \in X$ existe um $T_{\max } \leq \infty$ de modo que o problema abstrato (2.6) possui uma única solução fraca $U=U(t)$ definida no intervalo $\left[0, T_{\max }\right)$. Além disso, se $T_{\max }<\infty$,

$$
\lim _{t \rightarrow T_{\max }^{+}}\|U(t)\|=\infty .
$$

Em particular, se $F: X \rightarrow X$ é globalmente Lipschitz contínua, o problema abstrato

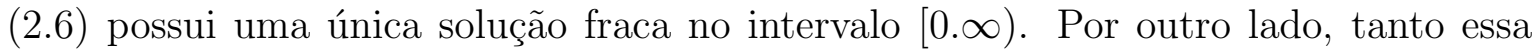
hipótese como a imposta no Teorema 2.13 sobre $F$ não são suficientes para garantir que a solução fraca possua mais regularidade. Os próximo teorema descreve os casos em que é possível uma solução mais regular.

Teorema 2.14. Sejam X espaço de Banach reflexivo e A gerador infinitesimal de um semigrupo de contrações. Seja $F: X \rightarrow X$ uma função localmente Lipschitz contínua e seja $U_{0} \in D(A)$. Então, a única solução fraca $U=U(t) \in C([0, T] ; X)$ do problema abstrato (2.6) em $[0, T]$ é uma solução forte do mesmo problema.

Se $X$ é um espaço de Banach e $A$ é um gerador infinitesimal de um $C_{0^{-}}$ semigrupo, o par $\left(D(A),\|\cdot\|_{A}\right)$ é um espaço de Banach, em que $\|x\|_{A}=\|x\|+\|A x\|$ é a norma do gráfico de $A$. Nos denotaremos por $Y=D(A)$ e enunciaremos o seguinte resultado:

Teorema 2.15. Seja $F: Y \rightarrow Y$ uma função Lipschitz contínua sobre $Y$. Se $U_{0} \in D(A)$, então o problema (2.6) possui uma única solução clássica.

Se, no teorema acima, assumirmos que $F: Y \rightarrow Y$ é localmente Lipschitz contínua em $Y$, aplicando o Teorema 2.13 para cada $x \in D(A)$, o P.V.I (2.6) possui uma única solução clássica no intervalo $\left[0, T_{\max }\right)$. Se $T_{\max }<\infty$,

$$
\lim _{t \rightarrow T_{\max }^{+}}(\|u(t)\|+\|A u(t)\|)=\infty
$$


Finalmente, apresentamos um resultado que mostra que as soluções clássicas do problema abstrato (2.6) forman um conjunto denso no espaço das soluções.

Teorema 2.16. Seja $f \in L^{1}(0, T ; X)$. Se U é uma solução fraca do problema abstrato (2.6) em $[0, T]$, então, para qualquer $\widetilde{T}<T$, U é limite uniforme em $[0, \widetilde{T}]$ de soluções clássicas do PVI (2.6).

\subsubsection{Atratores globais e dimensão fractal finita}

Faremos uma breve revisão da teoria de atratores globais, em sistemas dinâmicos de dimensão finita. As definições e teoremas que exporemos aqui serão utilizados nos capítulos posteriores. Para detalhes dos resultados que apresentaremos, indicamos as seguintes referências: Chueshov \& Lasiecka [10, 12, 13],

Babin \& Vishik [2], Eden et al [14], Hale [26], Pata \& Zelik [34], Temam [39] e Carvalho et al [6].

Denotaremos por $(X, d)$ um espaço métrico completo e por $\mathcal{C}(X)$ o conjunto das aplicações contínuas de $X$ em $X$.

Definição 2.9. Uma família de operadores $\{S(t)\}_{t \geq 0} \subset \mathcal{C}(X)$ é chamada de $C_{0}$ semigrupo não linear (ou simplesmente de $C_{0}$-semigrupo) se as seguintes condições forem satisfeitas:

i. $S(0)=I d: X \rightarrow X$ é o operador identidade.

ii. $S(t+s)=S(t) S(s)$ para cada $t, s \geq 0$.

iii. A aplicação $[0, \infty) \times X \ni(t, x) \mapsto S(t)(x) \in X$ é contínua para cada $x \in X$ fixado.

Ás vezes, o par $(X, S(t)), t \geq 0$, é também chamado de sistema dinâmico e $S(t)$ um semigrupo de evolução.

Definição 2.10. Seja $S(t): X \rightarrow X, t \geq 0$, um $C_{0}$-semigrupo. Diremos que um subconjunto $B \subset X$ é invariante (ou positivamente invariante) pelo semigrupo $S(t)$ quando $S(t) B=B$ para todo $t \geq 0($ ou $S(t) B \subset B)$. 
Definição 2.11. Seja $S(t): X \rightarrow X, t \geq 0$, um $C_{0}$-semigrupo. Um conjunto fechado e limitado $\mathcal{A} \subset X$ é chamado de atrator global para $S(t)$ se as seguintes propriedades são satisfeitas

i. $\mathcal{A}$ é um conjunto invariante por $S(t)$.

ii. $\mathcal{A}$ atrai (uniformemente) qualquer subconjunto limitado de $X$ sob a ação de $S(t)$, ou seja, para qualquer limitado $B \subset X$,

$$
\text { dist }_{X}(S(t) B, \mathcal{A}):=\sup _{x \in S(t) B} \inf _{y \in \mathcal{A}} d(x, y) \rightarrow 0 \quad \text { quando } t \rightarrow+\infty,
$$

em que dist ${ }_{X}(A, B)$ é chamada de semi-distância de Hausdorff entre os subconjuntos $A, B \subset X$.

Definição 2.12. Seja $S(t): X \rightarrow X, t \geq 0$, um $C_{0}$-semigrupo. Um conjunto $\mathfrak{B} \subset X$ é chamado de conjunto absorvente para $S(t)$ se, para qualquer subconjunto limitado $B \subset X$, existe $T_{0}=T_{0}(B) \geq 0$ tal que

$$
S(t) B \subset \mathfrak{B}, \quad \forall t \geq T_{0}
$$

Se $S(t)$ possui um conjunto absorvente limitado, diremos que o par $(X, S(t))$ é um sistema dinâmico dissipativo.

Definição 2.13. Um semigrupo $S(t): X \rightarrow X, t \geq 0$, é chamado assintoticamente suave se, para qualquer conjunto limitado e positivamente invariante $B \subset X$, existe um conjunto compacto $K \subset \bar{B}^{X}$ tal que

$$
\operatorname{dist}_{X}(S(t) B, K) \rightarrow 0 \text { quando } t \rightarrow+\infty
$$

De agora em diante, $\left(X,\|\cdot\|_{X}\right)$ é um espaço de Banach. Apresentaremos uma abordagem baseada nos trabalhos $[2,12,13,26,39]$, a qual, partindo dos conceitos acima, fornecerá resultados para os espaços de Banach.

Teorema 2.17. Seja $(X, S(t))$ um sistema dinâmico dissipativo definido sobre um espaço de Banach $X$. Então, $S(t)$ possui um atrator global compacto $\mathcal{A} \subset X$ se, e somente se, $S(t)$ é assintoticamente suave. 
O Teorema 2.17 nos dá condições suficientes para que um sistema dinâmico tenha um Atrator Global. Em resumo, precisamos de resultados que estipulem quando o semigrupo é assintoticamente suave. Antes de apresentarmos alguns resultados nesse sentido, enunciaremos o conceito de dimensão fractal.

Definição 2.14. Seja $\mathcal{A}$ um subconjunto compacto de um espaço de Banach $X$. A dimensão fractal de $\mathcal{A}$, denotada por $\operatorname{dim}_{f} \mathcal{A}$, é definida por

$$
\operatorname{dim}_{f} \mathcal{A}=\lim _{\varepsilon \rightarrow 0} \sup \frac{\ln \left(m_{\mathcal{A}}(\varepsilon)\right)}{\ln (1 / \varepsilon)}
$$

em que $m_{\mathcal{A}}(\varepsilon)$ é o número minimo de bolas fechadas de raio $\varepsilon$ que é necessário para cobrir $\mathcal{A}$.

Definição 2.15. Uma seminorma $n: X \rightarrow[0, \infty)$, definida sobre um espaço de Banach $X$, é chamada de compacta se, para qualquer limitado $B \subset X$, existe uma sequência $\left\{x_{n}\right\} \subset B$ tal que $n\left(x_{n}\right) \rightarrow 0$ quando $n \rightarrow \infty$.

Por fim, enunciaremos a definição de Atrator exponencial. Aqui, seguiremos mais de perto a abordagem de Chueshov \& Lasiecka [13]. Um dos textos clássicos nesse assunto é encontrado em Eden et al [14].

Definição 2.16. $\quad$ 1. Dizemos que um conjunto compacto $A_{\exp } \subset X$ é um atrator exponencial (ou conjunto inercial) para um sistema dinâmico $(X, S(t))$ se

a) $A_{\text {exp }}$ é um conjunto positivamente invariante e tem dimensão fractal finita.

b) Para todo conjunto limitado $B \subset X$, existem constantes positivas $t_{B}, C_{B}$ e $\gamma_{B}$ tais que

$$
\operatorname{dist}_{X}\left(S(t) B, A_{\text {exp }}\right)=\sup _{x \in B} \operatorname{dist}_{X}\left(S(t) x, A_{\text {exp }}\right) \leq C_{B} e^{-\gamma_{B}\left(t-t_{B}\right)}, t \geq t_{B}
$$

2. Diremos que $A_{\text {exp }} \subset X$ é um atrator exponencial generalizado se existe um expaço $\widetilde{X} \supset X$ tal que $A_{\exp }$ tem dimensão fractal finita em $\tilde{X}$. 


\subsubsection{Sistemas dinâmicos quase-estáveis}

Introduziremos o conceito de quase estabilidade para um sistema dinâmico $(H, S(t))$, em que $H$ é um espaço de Banach. Esse conceito nos fornece uma forma de reinterpretarmos os resultados da seção anterior para nosso contexto. Sejam $X, Y$ e $Z$ espaços de Banach reflexivos, com $X$ compactamente imerso em $Y$. Seja $H=X \times Y \times Z$, e consideremos o sistema dinâmico $(H, S(t))$ dado pelo operador de evolução

$$
S(t) z_{0}=\left(u(t), u_{t}(t), \theta(t)\right), \quad z_{0}=\left(u_{0}, u_{1}, \theta_{0}\right) \in H
$$

sendo $u$ e $\theta$ funções com a seguinte regularidade

$$
u \in C\left(\mathbb{R}^{+} ; X\right) \cap C^{1}\left(\mathbb{R}^{+} ; Y\right) \text { e } \theta \in C\left(\mathbb{R}^{+} ; Z\right) .
$$

Observação 2.5. As condições (2.8) e (2.9) nos dizem que nosso sistema dinâmico é obtido a partir das soluções de um sistema equações diferências parciais do tipo (1.1)(1.2).

Definição 2.17. Dizemos que o sistema dinâmico $(H, S(t))$ é quase estável sobre um conjunto $B \subset H$ se existe uma seminorma compacta $n_{X}(\cdot)$, definida sobre $X$ e funções escalares não negativas a $(t)$ e $c(t)$ localmente limitadas em $[0, \infty)$, e $b \in L^{\infty}\left(\mathbb{R}^{+}\right)$com $\lim _{t \rightarrow \infty} b(t)=0$, satisfazendo as seguintes desigualdades

$$
\left\|S(t) z_{0}^{1}-S(t) z_{0}^{2}\right\|_{H}^{2} \leq a(t)\left\|z_{0}^{1}-z_{0}^{2}\right\|_{H}^{2}
$$

$e$

$$
\left\|S(t) z_{0}^{1}-S(t) z_{0}^{2}\right\|_{H}^{2} \leq b(t)\left\|z_{0}^{1}-z_{0}^{2}\right\|_{H}^{2}+c(t) \sup _{0<s<t}\left[n_{X}\left(u^{1}(s)-u^{2}(s)\right)\right]^{2}
$$

em que $z^{i}(t)=\left(u^{i}(t), u_{t}^{i}(t), \theta^{i}(t)\right)$ com $z_{0}^{i}=z^{i}(0)=\left(u_{0}^{i}, u_{1}^{i}, \theta_{0}^{i}\right) \in B$ para $i=1,2$. A desigualdade (2.11) geralmente é chamada de "desigualdade de estabilização".

Os próximos resultados foram provados em Chueshov \& Lasiecka ([12], Theorem 7.9.6). Os teoremas a seguir garantem que um sistema dinâmico dissipativo e quase estável possui um atrator global com dimensão fractal finita. 
Proposição 2.1. Seja $(H, S(t))$ um sistema dinâmico nas condições dadas em (2.8) e (2.9). Assumimos que $(H, S(t))$ é quase estável para qualquer subconjunto limitado e positivamente invariante $\mathcal{B} \subset H$. Então, o sistema dinâmico $(H, S(t))$ é assintoticamente suave.

Um resultado imediato desta proposição é o seguinte corolário:

Corolário 2.1. Se $(H, S(t))$ é dissipativo e satisfaz as hipóteses da Proposição 2.1, então $(H, S(t))$ possui atrator global.

Por fim, a dimensão fractal finita é dada pelo seguinte teorema:

Teorema 2.18. Seja $(H, S(t))$ o sistema dinâmico descrito em (2.8), o qual satisfaz (2.9). Suponhamos que $(H, S(t))$ possui um atrator global $\mathcal{A}$ e que é quase estável sobre $\mathcal{A}$, então o atrator $\mathcal{A}$ tem dimensão fractal finita.

Na sequência, enunciaremos um resultado que garante a existência de atrator exponencial generalizado.

Teorema 2.19. Seja $(H, S(t))$ o sistema dinâmico é dado por (2.8) e (2.9). Suponhamos que $(H, S(t))$ é dissipativo quase estável sobre algum subconjunto absorvente limitado $\mathcal{B} \subset H$ e que existe um espaço de Banach $\widetilde{H} \supset H$, tal que $t \rightarrow S(t) y$ é $\alpha$-Hölder contínua em $\widetilde{H}$ para qualquer $y \in \mathcal{B}$. Ou seja, existem $0<\alpha \leq 1$ e uma constante $C_{\mathcal{B}, T}>0$ tal que

$$
\left\|S\left(t_{1}\right) y-S\left(t_{2}\right) y\right\|_{\widetilde{\mathcal{H}}} \leq C_{\mathcal{B}, T}\left|t_{1}-t_{2}\right|^{\alpha}, \quad t_{1}, t_{2} \in[0, T], \quad y \in \mathcal{B}
$$

Então o sistema dinâmico $(H, S(t))$ possui um atrator exponencial fractal generalizado com dimensão fractal finita em $\widetilde{H}$.

\subsubsection{Sistemas gradientes}

A estrutura geométrica do atrator global é um problema muito importante do ponto de vista das aplicações. Em geral, tal estrutura é muito complexa, mesmo no caso de um atrator de dimensão finita. Se o sistema dinâmico é gradiente, é possível 
mostrar que o atrator global, se existir, coincide com a variedade instável do conjunto de pontos de equilíbrio. Apresentamos nesta seção um resultado que nos possibilitará mostrar que nosso problema é gradiente. Para detalhes sobre a teoria de sistemas gradientes, sugerimos Chueshov \& Lasiecka $[12,13]$ e Carvalho et al [6].

Dado um sistema dinâmico $(X, S(t))$, podendo $X$ ser um espaço métrico, o conjunto das soluções estacionária (pontos de equilíbrio) é dado por

$$
\mathcal{N}=\{y \in X ; S(t) y=y, \quad \forall t \geq 0\}
$$

Definição 2.18. Seja $(X, S(t))$ um sistema dinâmico. A variedade instável do conjunto das soluções estacionárias $\mathcal{N}$, é o conjunto $\mathbb{M}^{u}(\mathcal{N})$ de todos os pontos $y \in X$ tais que existe um trajetória completa (solução global) $\gamma=\{u(t) ; t \in \mathbb{R}\}$ com a propriedade de que $u(0)=y e$

$$
\lim _{t \rightarrow-\infty} \operatorname{dist}_{X}(u(t), \mathcal{N})=0
$$

Observação 2.6. $\quad$ i. A variedade instável $\mathcal{M}^{u}(\mathcal{N})$ é um conjunto invariante em relação $a(X, S(t))$.

ii. Se o sistema dinâmico $(X, S(t))$ possui um atrator global $\mathcal{A}$, então $\mathbb{M}^{u}(\mathcal{N}) \subseteq \mathcal{A}$.

A igualdade $\mathbb{M}^{u}(\mathcal{N})=\mathcal{A}$ ocorre quando o sistema dinâmico é gradiente, os quais descrevemos na próxima definição:

Definição 2.19. Seja $Y \subseteq X$ um cojunto positivamente invariante em relação ao sistema dinâmico $(X, S(t))$.

i. Um funcional de Lyapunov para o sistema dinâmico $(X, S(t))$, é uma função $\Phi$ : $X \rightarrow \mathbb{R}$, tal que a aplicação $t \rightarrow \Phi(S(t) y)$, para cada $y \in Y$, é não-crescente.

ii. O funcional de Lyapunov $\Phi(y)$ é dito ser estrito sobre $Y$, se a equação $\Phi(S(t) y)=S(y)$, para algum $y \in Y$, implicar que $S(t) y=y$, isto é, $y \in \mathcal{N}$

iii. O sistema dinâmico $(X, S(t))$ é gradiente, se existe um funcional de Lyapynov estrito sobre o espaço $X$. 
Finalmente, estamos pronto para enunciar os seguintes resultados:

Teorema 2.20. Suponhamos que o sistema dinâmico $(X, S(t))$ possui um atrator global $\mathcal{A}$. $S e(X, S(t))$ possui um funcional Lyapunov estrito sobre $\mathcal{A}$, então $\mathcal{A}=\mathbb{M}^{u}(\mathcal{N})$. Além disso, o atrator $\mathcal{A}$ é o conjunto de todas as trajetórias completas $\gamma=\{u(t) ; t \in \mathbb{R}\}$ tais que

$$
\lim _{t \rightarrow-\infty} \operatorname{dist}_{X}(u(t), \mathcal{N})=0 \text { e } \lim _{t \rightarrow \infty} \operatorname{dist}_{X}(u(t), \mathcal{N})=0 .
$$

Corolário 2.2. Seja $(X, S(t))$ um sistema dinâmico gradiente e assintoticamente suave. Assuma que o funcional de Lyapunov $\Phi(x)$ é limitado sobre cada conjunto limitado de $X$ e que o conjunto $\Phi_{R}=\{x ; \Phi(x) \leq R\}$ é limitado para cada $R>0$. Se o conjunto $\mathcal{N}$ das soluções estacionárias é limitado então $(X, S(t))$ possui um atrator um atrator global compacto

$$
\mathcal{A}=\mathbb{M}^{u}(\mathcal{N})
$$

Com todos os resultados acima em mãos, seremos capazes de provar a existência tanto de um atrator global com dimensão fractal finita quanto do atrator exponencial fractal generalizado, tomando como base os trabalhos de Chueshov \& I. Lasiecka [12]. Assim, todos os nossos esforços estarão focados em encontrar resultados que garantam que nosso sistema dinâmico é da forma dada em (2.8) e (2.9), dissipativo e quase etável. 



\section{Capítulo 3}

\section{Boa colocação no sentido de}

\section{Hadamard}

Seja $\Omega$ um domínio limitado de $\mathbb{R}^{2}$ com fronteira suave $\Gamma=\partial \Omega$. Neste Capítulo, estudaremos a existência, unicidade e continuidade em relação aos dados iniciais para o problema

$$
\begin{aligned}
& u_{t t}+\Delta^{2} u-M\left(\int_{\Omega}|\nabla u|^{2} d x\right) \Delta u-\Delta u_{t t}+f(u)+\nu \Delta \theta=h(x), \text { em } \Omega \times \mathbb{R}^{+} \\
& \theta_{t}-\omega \Delta \theta-(1-\omega) \int_{0}^{\infty} k(s) \Delta \theta(t-s) d s-\nu \Delta u_{t}=0, \text { em } \Omega \times \mathbb{R}^{+}
\end{aligned}
$$

com condições de fronteira

$$
u=\Delta u=0, \quad \theta=0 \text { em } \Gamma \times \mathbb{R}^{+},
$$

e condições iniciais

$$
u(x, 0)=u_{0}(x), \quad u_{t}(x, 0)=u_{1}(x),\left.\quad \theta(x, t)\right|_{t \leq 0}=\theta_{0}(x,-t) \quad \text { em } \quad \Omega .
$$

O problema (3.1)-(3.4) não corresponde a um sistema dinâmico autônomo. Assim, para que possamos utilizar a teoria de semigrupos, procederemos como em Giorgi, Marzotti \& Pata [17], Giorgi \& Pata [18] e Giorgi, Grasseli \& V. Pata [19]. Para isso, definimos uma nova variável $\eta=\eta^{t}(x, s)$ por 


$$
\eta^{t}(s)=\int_{0}^{s} \theta(t-y) d y, \quad(t, s) \in \times[0, \infty) \times \mathbb{R}^{+}
$$

Observamos que

$$
\begin{aligned}
& \frac{\partial}{d t} \eta^{t}(s)=\frac{\partial}{d t} \int_{t-s}^{t} \theta(y) d y=\theta(t)-\theta(t-s) \\
& \mathrm{e} \\
& \frac{\partial}{d s} \eta^{t}(s)=\theta(t-s) .
\end{aligned}
$$

Logo, a variável $\eta$ satisfaz formalmente a equação

$$
\eta_{t}^{t}+\eta_{s}^{t}=\theta, \quad \text { em } \Omega, \quad(t, s) \in \mathbb{R} \times \mathbb{R}
$$

com condição inicial

$$
\eta^{t}(0)=0, \text { em } \Omega, t \geq 0
$$

Suponhamos que $k \in C^{1}(\mathbb{R})$ é uma função positiva, convexa e decrescente,

então

$$
\begin{aligned}
\frac{\partial}{d s}\left\{k(s) \Delta \eta^{t}(s)\right\} & =k^{\prime}(s) \Delta \eta^{t}(s)+k(s) \frac{\partial}{d s} \Delta \eta^{t}(s) \\
& =k^{\prime}(s) \Delta \eta^{t}(s)+k(s) \Delta \theta(t-s)
\end{aligned}
$$

Assim, integrando por partes em $s$ de 0 até $\infty$, temos

$$
\int_{0}^{\infty} k(s) \Delta \theta(t-s) d s=-\int_{0}^{\infty} k^{\prime}(s) \Delta \eta^{t}(s) d s
$$

Definimos então

$$
\mu(s)=-(1-\omega) k^{\prime}(s) .
$$

Mais a frente, faremos algumas hipóteses sobre a função $\mu$.

Podemos, assim, reescrever (3.1)-(3.4) como segue

$$
\begin{aligned}
& u_{t t}+\Delta^{2} u-M\left(\|\nabla u\|_{2}^{2}\right) \Delta u-\Delta u_{t t}+f(u)+\nu \Delta \theta=h(x) \\
& \theta_{t}-\omega \Delta \theta-\int_{0}^{\infty} \mu(s) \Delta \eta^{t}(s) d s-\nu \Delta u_{t}=0 \\
& \eta_{t}^{t}+\eta_{s}^{t}=\theta(t)
\end{aligned}
$$

com condições iniciais 


$$
u(x, 0)=u_{0}(x), u_{t}(x, 0)=u_{1}(x), \theta(x, \tau)=\theta_{0}(x, \tau) \text { e } \eta^{0}(x, s)=\eta_{0}(x, s),
$$

em que

$$
u_{0}(x)=u_{0}(x, 0), u_{1}(x)=\partial_{t} u_{0}(x, 0), \theta_{0}(x)=\theta_{0}(x, 0)
$$

e

$$
\eta_{0}(s)=\eta^{0}(s)=\int_{0}^{s} \theta(-y) d y .
$$

Além disso, $\eta^{t}(0)=0$, em $\Omega$ e $t \geq 0$. Ainda, temos as seguintes condições de fronteira

$$
\begin{aligned}
& u=\Delta u=0, \text { em } \Gamma \times \mathbb{R}^{+}, \\
& \theta=0 \text { em } \Gamma \times \mathbb{R}^{+}, \\
& \eta^{t}(s)=0 \text { em } \Gamma \times \mathbb{R}^{+} \times \mathbb{R}^{+} .
\end{aligned}
$$

Assim, nosso problema será doravante (3.6)-(3.9).

\subsection{Hipóteses e notações}

Assumiremos que $M \in C(\mathbb{R})$ e que existem constantes $\alpha, c_{M} \geq 0$ tais que

$$
\alpha<\sqrt{\lambda_{1}} \text { e } \mathcal{M}(s) \geq-\alpha s-c_{M}
$$

e

$$
M(s) s \geq \mathcal{M}(s)-\frac{\alpha}{2} s-c_{M}
$$

em que $\mathcal{M}(t)=\int_{0}^{t} M(s) d s$ e $\lambda_{1}$ é o primeiro autovalor do operador $\Delta$ com condições de fronteira $u=0$ em $\Gamma$. Suponhamos $\mu \in C^{1}\left(\mathbb{R}_{+}\right) \cap L^{1}\left(\mathbb{R}_{+}\right)$e que existem constantes positivas $\gamma_{0}, k_{0}$ e $k_{1}$ tais que

$$
\begin{array}{ll}
0<\mu(s)<\infty, & \mu^{\prime}(s) \leq 0, \\
\mu^{\prime}(s)+\gamma_{0} \mu(s) \leq 0 & \forall s \in \mathbb{R}^{+},
\end{array}
$$

e

$$
k_{0}=\int_{0}^{\infty} \mu(s) d s, \quad k_{1}=\int_{0}^{\infty} \mu^{\prime}(s) d s
$$


Em relação a $f: \mathbb{R} \rightarrow \mathbb{R}$, fazemos as seguintes afirmações

$$
f \in C^{1}(\mathbb{R}), \quad|f(u)| \leq\left(1+|u|^{r}\right)
$$

para algum $r>0$. Além disso, assumiremos

$$
f(u) u \geq \widehat{f}(u) \geq-\rho, \forall u \in \mathbb{R}
$$

em que $\rho>0$ e $\widehat{f}(z)=\int_{0}^{z} f(s) d s$.

Fixaremos a seguinte notação para nossos espaços de Sobolev

$$
V_{0}=L^{2}(\Omega), \quad V_{1}=H_{0}^{1}(\Omega), \quad V_{2}=H^{2}(\Omega) \cap H_{0}^{1}(\Omega)
$$

e

$$
V_{3}=\left\{u \in H^{3}(\Omega) \mid u=\Delta u=0 \text { em } \Gamma\right\}
$$

cada um munido com as seguintes normas $\|\cdot\|_{V_{i}}$, para $i=0,1,2,3$. Para a variável $\eta$, definimos

$$
\mathcal{M}_{1}=L_{\mu}^{2}\left(\mathbb{R}^{+} ; V_{1}\right)=\left\{\eta: \mathbb{R}^{+} \rightarrow V_{1} ; \int_{0}^{\infty} \mu(s)\|\eta(s)\|_{V_{1}}^{2} d s\right\},
$$

munidos com o produtos interno e norma

$$
\left(\eta_{1}, \eta_{2}\right)_{\mu, 1}=\int_{0}^{\infty} \mu(\tau)\left(\eta_{1}(\tau), \eta_{2}(\tau)\right)_{V_{1}} d \tau, \quad\|\eta\|_{\mu, 1}^{2}=\int_{0}^{\infty} \mu(\tau)\|\eta(\tau)\|_{V_{1}}^{2} d \tau
$$

Com essa notação, nosso espaço de fase é

$$
\mathcal{H}_{0}=V_{2} \times V_{1} \times V_{0} \times \mathcal{M}_{1}
$$

Denotaremos o produto interno e sua respectiva norma de $\mathcal{H}_{0}$ por

$$
\left\langle U_{1}, U_{2}\right\rangle_{\mathcal{H}_{0}}=\left(\Delta u_{1}, \Delta u_{2}\right)+\left(\nabla v_{1}, \nabla v_{2}\right)+\left(\theta_{1}, \theta_{2}\right)+\left(\eta_{1}, \eta_{2}\right)_{\mu, 1}
$$

e

$$
\left\|U_{1}\right\|_{\mathcal{H}_{0}}^{2}=\left\|\Delta u_{1}\right\|_{2}^{2}+\left\|\nabla v_{1}\right\|_{2}^{2}+\left\|\left.\theta_{1}\right|_{2} ^{2}+\right\| \eta_{1} \|_{\mu, 1}^{2},
$$

em que $U_{i}=\left(u_{i}, v_{i}, \theta_{i}, \eta_{i}\right) \in \mathcal{H}_{0}, \operatorname{com} i=1,2$. 


\subsection{Existência, unicidade e dependência contínua}

Nesta seção, mostraremos, através da teoria de semigrupos, que o problema (3.6)-(3.9) é bem posto. Podemos descrever a derivada $\eta_{s}^{t}$ na forma de operador. Seguindo Grasselli \& Pata [22], definimos

$$
\mathcal{T} \eta=-\eta_{s}, \quad \eta \in D(\mathcal{T})
$$

com domínio

$$
D(\mathcal{T})=\left\{\eta \in \mathcal{M}_{1} ; \quad \eta_{s} \in \mathcal{M}_{1}, \quad \eta(0)=0\right\}
$$

O operador $\mathcal{T}$ é um gerador infinitesimal de um $C_{0}$-semigrupo de contrações sobre $\mathcal{M}_{1}$, ver Grasselli \& Pata [22] e Giorgi \& Pata. Além disso, temos :

Proposição 3.1. O operador $\mathcal{T}$ satisfaz as seguintes relações

$i .(\mathcal{T} \eta, \eta)_{\mu, 1}=\frac{1}{2} \int_{0}^{\infty} \mu^{\prime}(s)\|\nabla \eta\|_{2}^{2} d s$, para $\eta \in D(\mathcal{T}) ;$

ii. $(\mathcal{T} \eta, \eta)_{\mu, 1} \leq-\frac{\gamma_{0}}{2}\|\eta\|_{\mu, 1}^{2}$ para $\eta \in D(\mathcal{T})$.

Demonstração. De fato, integrando por partes na variável $s$ e utilizando as hipóteses (3.13) sobre $\mu$, temos

$$
\begin{aligned}
(\mathcal{T} \eta, \eta)_{\mu, 1}=\int_{0}^{\infty} \mu(s)\left(-\nabla \eta_{s}, \nabla \eta\right) d s & =-\left.\frac{1}{2} \mu(s)\|\nabla \eta\|_{2}^{2}\right|_{0} ^{\infty}+\frac{1}{2} \int_{0}^{\infty} \mu^{\prime}(s)\|\nabla \eta\|_{2}^{2} d s \\
& =\frac{1}{2} \int_{0}^{\infty} \mu^{\prime}(s)\|\nabla \eta\|_{2}^{2} d s
\end{aligned}
$$

o que demonstra o primeiro item. Para demonstrar o segundo, basta notar, a partir da desigualdade $\mu^{\prime}(s)+\gamma_{0} \mu(s) \leq 0$, que

$$
\frac{1}{2} \int_{0}^{\infty} \mu^{\prime}(s)\|\nabla \eta\|_{2}^{2} d s \leq-\frac{\gamma_{0}}{2} \int_{0}^{\infty} \mu(s)\|\nabla \eta\|_{2}^{2} d s=-\frac{\gamma_{0}}{2}\|\eta\|_{\mathcal{M}_{\mu, 1}}^{2}
$$


No problema (3.6)-(3.9), denotamos $v=u_{t}$. Com isso, observamos que

$$
\begin{aligned}
& u_{t}=v \\
& (I d-\Delta) v_{t}=-\Delta^{2} u+M\left(\|\nabla u\|_{2}^{2}\right) \Delta u-f(u)-\nu \Delta \theta+h(x) \\
& \theta_{t}=\omega \Delta \theta+\int_{0}^{\infty} \mu(s) \Delta \eta^{t}(s) d s+\nu \Delta v \\
& \eta_{t}^{t}=\mathcal{T} \eta^{t}+\theta .
\end{aligned}
$$

Sejam

$$
U(t)=\left(u(t), v(t), \theta(t), \eta^{t}\right)^{T}
$$

e

$$
\mathcal{L}=\left[\begin{array}{cccc}
0 & I d & 0 & 0 \\
-(I d-\Delta)^{-1} \Delta^{2} & 0 & -\nu(I d-\Delta)^{-1} \Delta & 0 \\
0 & \nu \Delta & \omega \Delta & B_{\mu} \\
0 & 0 & I d & \mathcal{T}
\end{array}\right]
$$

em que

$$
B_{\mu} \eta=\int_{0}^{\infty} \mu(s) \Delta \eta(s) d s
$$

Denotaremos por

$$
F(U(t))=\left[\begin{array}{ll}
M\left(\|\nabla u(t)\|_{2}^{2}\right)(I d-\Delta)^{-1} \Delta u(t) & -(I d-\Delta)^{-1}(f(u(t))-h) \\
& 0 \\
& 0
\end{array}\right]
$$

Com a notação acima, definimos o operador

$$
\mathcal{L}: D(\mathcal{L}) \subset \mathcal{H}_{0} \rightarrow \mathcal{H}_{0}
$$

com domínio 


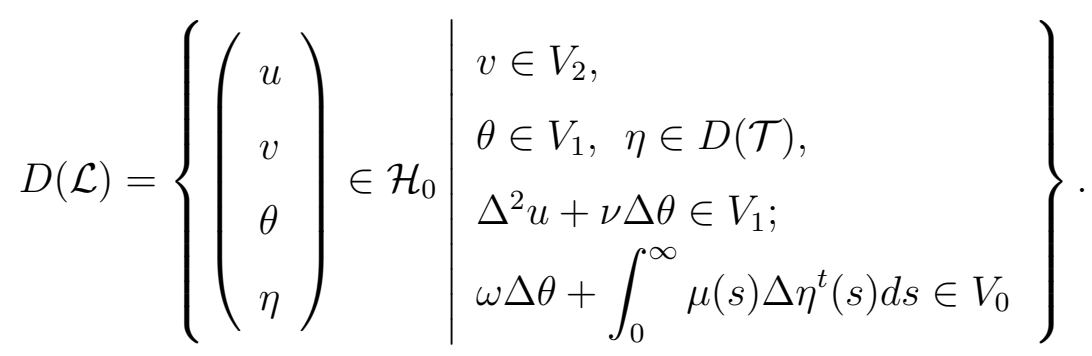

Como em Giorgi \& Pata [18] e Grasselli \& Squassina [24], mostraremos, atráves da teoria de semigrupos, que o problema

$$
\left\{\begin{array}{l}
\dot{U}(t)=\mathcal{L} U(t)+F(U(t)) \\
U(0)=U_{0} \in \mathcal{H}_{0}
\end{array}\right.
$$

possui uma única solução fraca. Neste caso, em que $U_{0}=\left(u_{0}, u_{1}, \theta_{0}, \eta_{0}\right) \in \mathcal{H}_{0}$, observamos que

$$
\mathcal{L} U(t)=\left[\begin{array}{c}
v \\
-(I d-\Delta)^{-1} \Delta^{2} u-\nu(I d-\Delta)^{-1} \Delta \theta \\
\omega \Delta \theta+\int_{0}^{\infty} \mu(s) \Delta \eta(s) d s+\nu \Delta v \\
\mathcal{T} \eta^{t}+\theta
\end{array}\right]
$$

Nosso principal resultado desse capítulo é dado no teorema abaixo:

Teorema 3.1. Suponhamos que as hipóteses (3.11)-(3.16) sejam satisfeitas e que $h \in$ $L^{2}(\Omega)$.

i. Então, para todo $U_{0} \in \mathcal{H}_{0}$ e $T>0$, existe uma uma única solução fraca do problema (3.25) $U(t) \in C\left(0, T ; \mathcal{H}_{0}\right)$, tal que

$$
U(t)=e^{t \mathcal{L}} U_{0}+\int_{0}^{t} e^{(t-s) \mathcal{L}} F(U(s)) d s, \quad t \in[0, T] .
$$

Além disso, se $U_{0} \in D(\mathcal{L}), U(t)$ é uma solução clássica.

ii. Se $U_{1}, U_{2} \in \mathcal{H}_{0}$, existe uma constante positiva $\alpha$ tal que as respectivas soluções fracas $U_{1}(t)$ e $U_{2}(t)$ satisfazem

$$
\left\|U_{1}(t)-U_{2}(t)\right\|_{\mathcal{H}_{0}} \leq e^{\varrho t}\left\|U_{1}-U_{2}\right\|_{\mathcal{H}_{0}}, \quad t \in[0, T]
$$




\subsubsection{Existência local de soluções}

Nesta subseção, mostraremos que o problema (3.25) possui uma única solução local.

Lema 3.1. Suponhamos as mesmas condições do Teorema 3.1. Então o operador $\mathcal{L} e ́$ gerador infinitesimal de um $C_{0}$-semigrupo de contrações.

Demonstração. A fim de mostrar que o operador $\mathcal{L}$ é um gerador infitesimal de um semigrupo de contrações, podemos, por um instante, supor que $M=F=h=0$. A partir das equações do problema (3.6)-(3.9), observamos, utilizando o método de energia, que

$$
\langle\mathcal{L} U(t), U(t)\rangle_{\mathcal{H}_{0}}=-\omega\|\nabla \theta(t)\|_{2}^{2}+\frac{1}{2} \int_{0}^{\infty} \mu^{\prime}(s)\left\|\nabla \eta^{t}(s)\right\|^{2} d s \leq 0 .
$$

Concluímos, a partir das hipóteses sobre $\mu$ dada em (3.13), que

$$
\langle\mathcal{L} U(t), U(t)\rangle_{\mathcal{H}_{0}} \leq 0, \quad \forall t \geq 0
$$

o que prova que $\mathcal{L}$ é dissipativo. Demonstraremos agora que $\mathcal{L}$ é maximal. De fato, dado $G=\left(u^{*}, v^{*}, \theta^{*}, \eta^{*}\right) \in \mathcal{H}_{0}$, devemos mostrar que existe uma única solução $U$ de $(I d-\mathcal{L}) U=G$ em $D(\mathcal{L})$. Isto é, demonstraremos que o sistema

$$
\begin{aligned}
& u-v=u^{*} ; \\
& v+(I d-\Delta)^{-1} \Delta^{2} u+\nu(I d-\Delta)^{-1} \Delta \theta=v^{*} ; \\
& \theta-\omega \Delta \theta-B_{\mu} \eta-\nu \Delta v=\theta^{*} ; \\
& \eta-\mathcal{T} \eta-\theta=\eta^{*} .
\end{aligned}
$$

possui uma única solução $U(t)=\left(u(t), v(t), \theta(t), \eta^{t}\right) \in D(\mathcal{L})$. As igualdades $(3.30)$ e (3.33) levam a

$$
u=v+u^{*}
$$

e

$$
n(s)=\left(1-e^{-s}\right) \theta-\int_{0}^{s} e^{\tau-s} \eta^{*}(\tau) d \tau
$$


respectivamente. Substituindo (3.35) em (3.32), obtemos

$$
\begin{aligned}
\theta^{*} & =\theta-\nu \Delta v-\omega \Delta \theta-B_{\mu} \eta=\nu \Delta v-\omega \Delta \theta-B_{\mu}\left(\left(1-e^{-s}\right) \theta-\int_{0}^{s} e^{\tau-s} \eta^{*}(\tau) d \tau\right) \\
& =\nu \Delta v-\left(\omega+c_{\mu}\right) \Delta \theta+\int_{0}^{\infty} \mu(s) \int_{0}^{s} e^{\tau-s} \Delta \eta^{*}(\tau) d \tau d s .
\end{aligned}
$$

Logo, temos

$$
\theta-\nu \Delta v-\left(\omega+c_{\mu}\right) \Delta \theta=\theta^{*}-\int_{0}^{\infty} \mu(s) \int_{0}^{s} e^{\tau-s} \Delta \eta^{*}(\tau) d \tau d s
$$

em que $c_{\mu}=\int_{0}^{\infty}\left(1-e^{-s}\right) \mu(s) d s$

Precisamos mostrar que o lado direito de (3.36) é bem definido, isto é, pertence a $V_{-1}$, o espaço dual de $V_{1}$. Empregaremos um argumento similar ao utilizado por Grasselli \& Pata [22]. Seja $\widetilde{\eta} \in V_{1}$, tal que $\|\widetilde{\eta}\|_{V_{1}}=1$. Então

$$
\begin{aligned}
\left|\left\langle\int_{0}^{\infty} \mu(y) \int_{0}^{y} e^{\tau-y} \Delta \eta^{*}(\tau) d \tau d y, \widetilde{\eta}\right\rangle\right| & =\left|\int_{0}^{\infty} \mu(y) \int_{0}^{y} e^{\tau-y}\left(\nabla \eta^{*}(\tau), \nabla \widetilde{\eta}\right)_{V_{0}} d \tau d y\right| \\
& \leq \int_{0}^{\infty} \mu(y)^{1 / 2}\|\widetilde{\eta}\|_{V_{1}} \mu(y)^{1 / 2} \int_{0}^{s} e^{\tau-y}\left\|\eta^{*}(\tau)\right\|_{V_{1}} d \tau d y \\
& \leq k_{0}\left\|\eta^{*}\right\|_{\mathcal{M}_{1}},
\end{aligned}
$$

em que aplicamos o seguinte resultado para obter a ultima desigualdade:

Se $f, g \in L^{2}\left(\mathbb{R}^{+}\right)$e $h \in L^{1}\left(\mathbb{R}^{+}\right)$, então

$$
\left|\langle f, g * h\rangle_{L^{2}\left(\mathbb{R}^{+}\right)}\right| \leq\|f\|_{L^{2}\left(\mathbb{R}^{+}\right)}\|g\|_{L^{2}\left(\mathbb{R}^{+}\right)}\|h\|_{L^{1}\left(\mathbb{R}^{+}\right)} .
$$

Portanto

$$
\left\|\int_{0}^{\infty} \mu(s) \int_{0}^{s} e^{\tau-s} \Delta \eta^{*}(\tau) d \tau d s\right\|_{V_{-1}} \leq k_{0}\left\|\eta^{*}\right\|_{\mathcal{M}_{1}} .
$$

Consequentemente, o lado direito de (3.36) é um funcional limitado sobre $V_{1}$.

As equações (3.31) e (3.34) nos fornecem

$$
(I d-\Delta) v^{*}=(I d-\Delta) v+\Delta^{2}\left(u+u^{*}\right)+\nu \Delta \theta .
$$


Logo,

$$
v-\Delta v+\Delta^{2} v+\Delta \theta=(I d-\Delta) v^{*}-\Delta^{2} u^{*}
$$

O lado direito de (3.37) é um funcional linear limitado sobre $V_{2}$. Juntando (3.36) e (3.37), temos o sistema

$$
\begin{aligned}
& \theta-\nu \Delta v-\beta \Delta \theta=v^{* *} \\
& v-\Delta v+\Delta^{2} v+\nu \Delta \theta=u^{* *}
\end{aligned}
$$

em que

$$
\begin{gathered}
v^{* *}=\theta^{*}-\int_{0}^{\infty} \mu(s) \int_{0}^{s} e^{\tau-s} \Delta \eta^{*}(\tau) d \tau d s \in V_{-1}, \\
u^{* *}=(I d-\Delta) v^{*}-\Delta^{2} u^{*} \in V_{-2}
\end{gathered}
$$

e $\beta=\omega+c_{\mu}$, em que $V_{-2}$ é o dual de $V_{2}$. Definimos o funcional $C: V \times V \rightarrow \mathbb{R}$,

$$
\begin{aligned}
C\left(\left(v_{1}, \theta_{1}\right),\left(v_{2}, \theta_{2}\right)\right)= & \left(v_{1}, v_{2}\right)+\left(\theta_{1}, \theta_{2}\right)+\left(\nabla v_{1}, \nabla v_{2}\right)+\beta\left(\nabla \theta_{1}, \nabla \theta_{2}\right) \\
& +\left(\Delta v_{1}, \Delta v_{2}\right)-\nu\left(\nabla \theta_{1}, \nabla v_{2}\right)+\nu\left(\nabla v_{1}, \nabla \theta_{2}\right),
\end{aligned}
$$

em que $V=V_{2} \times V_{1}$ e $V^{\prime}=V_{-2} \times V_{-1}$ é o dual de $V$ em relação a $H=V_{0} \times V_{0}$.

Claramente, $C$ é uma forma bilinear limitada e coerciva. Observamos que $f=\left(u^{* *}, v^{* *}\right)$ é um funcional limitado sobre $V$. Pelo teorema de Lax-Milgram, existe um único $\operatorname{par}(v, \theta) \in V$ tal que, para todo $\operatorname{par}(\widetilde{v}, \widetilde{\theta}) \in V$

$$
C((v, \theta),(\widetilde{v}, \widetilde{\theta}))=\left\langle\left(u^{* *}, v^{* *}\right),(\widetilde{v}, \widetilde{\theta})\right\rangle, \quad \forall(\widetilde{v}, \widetilde{\theta}) \in V
$$

em que $\langle\cdot, \cdot\rangle$ é a dualidade entre $V$ e $V^{\prime}$. Assim, concluimos que existem únicos $v \in V_{2}$ e $\theta \in V_{1}$ solução do sistema (3.38)-(3.39).

Observamos que

$$
\begin{aligned}
I d-\Delta: & V_{1} \longrightarrow V_{-1} \\
& z \longrightarrow(I d-\Delta) z
\end{aligned}
$$


é uma bijeção isométria. Então, se $z \in V_{1}$ devemos ter $(I d-\Delta) z \in V_{-1}$. Por outro lado, segue da equação (3.31) que

$$
(I d-\Delta)^{-1} \Delta^{2} u+\nu(I d-\Delta)^{-1} \Delta \theta \in V_{-1},
$$

o que nos leva a concluir que $\Delta^{2} u+\nu \Delta \theta \in V_{1}$.

O mesmo procedimento utilizado para mostrar que o lado direto da equação (3.36 ) é bem definido, mostra que

$$
n(s)=\left(1-e^{-s}\right) \theta-\int_{0}^{s} e^{\tau-s} \eta^{*}(\tau) d \tau
$$

$\eta$ é bem definida e que $\eta \in \mathcal{M}_{1}$. Mostraremos a seguinte afirmação: $\eta_{s} \in \mathcal{M}_{1}$ se $\eta \in \mathcal{M}_{1}$. De fato, supomos que $\eta \in \mathcal{M}_{1}$. Como $\theta \in V_{1}$, então podemos provar que $\left(1-e^{-s}\right) \theta \in \mathcal{M}_{1}$. Assim,

$$
\int_{0}^{s} e^{\tau-s} \eta^{*}(\tau) d \tau=n(s)-\left(1-e^{-s}\right) \theta \in \mathcal{M}_{1}
$$

Por outro lado,

$$
\eta_{s}(s)=e^{-s} \theta-\eta^{*}(s)+\int_{0}^{s} e^{y-s} \eta^{*}(y) d y .
$$

Segue de (3.43) que $\eta_{s} \in \mathcal{M}_{1}$. Consequentemente, $\eta \in D(\mathcal{T})$. Resta verificar que

$$
\omega \Delta \theta+\int_{0}^{\infty} \mu(s) \Delta \eta^{t}(s) d s \in V_{0}
$$

A partir de (3.32), temos

$$
-\omega \Delta \theta-\int_{0}^{\infty} \mu(s) \Delta \eta^{t}(s) d s=\theta^{*}+\nu \Delta v-\theta \in V_{0}
$$

o que mostra (3.45).

Portanto, o operador $\mathcal{L}$ é maximal. Pelo Teorema 2.12 (teorema de Lumer-Phillips), concluímos que $\mathcal{L}$ é um gerador infinitesimal de um $C^{0}$-semigrupo de contrações. 
Lema 3.2. A aplicação $F$ definida em (3.23) é localmente Lipschitz em $\mathcal{H}_{0}$, ou seja, existe uma constante $L_{0 M f}$, que depende dos dados iniciais e das funções $M$ e $f$, tal que

$$
\left\|F\left(U_{1}\right)-F\left(U_{2}\right)\right\|_{\mathcal{H}_{0}} \leq L_{0 M f}\left\|U_{1}-U_{2}\right\|_{\mathcal{H}_{0}}
$$

em que $L_{0 M f}=\max \left\{1, C_{0 f}, C_{0 M}\right\}$, sendo $C_{0 f}$ e $C_{0 M}$ são constantes que dependem de f e $M$ respectivamente.

Demonstração. Lembramos um resultado bem coonhecido,

$$
\begin{aligned}
I d-\Delta: & V_{1} \longrightarrow V_{-1} \\
& z \longrightarrow(I d-\Delta) z
\end{aligned}
$$

é uma bijeção isométria. Então, $\|z\|_{V_{1}}=\|(I d-\Delta) z\|_{V_{-1}}$, o que nos leva a $\left\|(I d-\Delta)^{-1} z\right\|_{V_{1}}=\|z\|_{V_{-1}}$. Desta forma,

$$
\begin{aligned}
\left\|F\left(U_{1}\right)-F\left(U_{2}\right)\right\|_{\mathcal{H}_{0}} & =\left\|(I d-\Delta)^{-1}\left[M\left(\|\nabla u\|_{2}^{2}\right) \Delta u_{1}-M\left(\|\nabla u\|_{2}^{2}\right) \Delta u_{2}-\left(f\left(u_{1}\right)-f\left(u_{2}\right)\right)\right]\right\|_{V_{1}} \\
& \leq\left\|M\left(\|\nabla u\|_{2}^{2}\right) \Delta u_{1}-M\left(\|\nabla u\|_{2}^{2}\right) \Delta u_{2}\right\|_{V_{-1}}+\left\|f\left(u_{1}\right)-f\left(u_{2}\right)\right\|_{V_{-1}}
\end{aligned}
$$

Por outro lado, dado $w \in V_{1}$, segue das hipóteses (3.15)-(3.16) e do Teorema do Valor Médio que

$$
\left|\left\langle f\left(u_{1}\right)-f\left(u_{2}\right), w\right\rangle\right| \leq \int_{\Omega}\left|f\left(u_{1}\right)-f\left(u_{2}\right)\right||w| d x \leq C_{0 f}\left\|u_{1}-u_{2}\right\|_{V_{1}}\|w\|_{V_{1}},
$$

em que $C_{0 f}>0$ é uma constante que depende dos dados iniciais. Portanto

$$
\left\|f\left(u_{1}\right)-f\left(u_{2}\right)\right\|_{V_{-1}} \leq C_{0 f}\left\|\nabla u_{1}-\nabla u_{2}\right\|_{2}
$$

Novamente, pelo Teorema do Valor Médio e as hipóteses (3.11)-(3.12), existe uma constante $C_{M}>0$ tal que

$$
\begin{aligned}
& M\left(\left\|\nabla u_{1}\right\|_{2}^{2}\right)-M\left(\left\|\nabla u_{2}\right\|_{2}^{2}\right) \leq C_{M}\left(\left\|\nabla u_{1}\right\|_{2}^{2}-\left\|\nabla u_{2}\right\|_{2}^{2}\right) \\
& =C_{M}\left(\left\|\nabla u_{1}\right\|_{2}+\left\|\nabla u_{2}\right\|_{2}\right)\left(\left\|\nabla u_{1}\right\|_{2}-\left\|\nabla u_{2}\right\|_{2}\right) \leq C_{0 M}\left\|\nabla u_{1}-\nabla u_{2}\right\|_{2},
\end{aligned}
$$


em que $C_{0 M}>0$, é uma constante dependente dos dados iniciais. Logo, para $w \in V_{1}$

$$
\left|\left\langle M\left(\left\|\nabla u_{1}\right\|_{2}^{2}\right) \Delta u_{1}-M\left(\left\|\nabla u_{2}\right\|_{2}^{2}\right) \Delta u_{2}, w\right\rangle\right| \leq C_{M, R}\left\|\nabla u_{1}-\nabla u_{2}\right\|_{2}\|w\|_{V_{1}} .
$$

Então

$$
\left\|M\left(\left\|\nabla u_{1}\right\|_{2}^{2}\right) \Delta u_{1}-M\left(\left\|\nabla u_{2}\right\|_{2}^{2}\right) \Delta u_{2}\right\|_{V_{-1}} \leq C_{0 M}\left\|\nabla u_{1}-\nabla u_{2}\right\|_{2} .
$$

Combinando as desigualdades (3.48) e (3.49) teremos

$$
\left\|F\left(U_{1}\right)-F\left(U_{2}\right)\right\|_{\mathcal{H}_{0}} \leq \max \left\{1, C_{0 f}, C_{0 M}\right\}\left\|U_{1}-U_{2}\right\|_{\mathcal{H}_{0}} .
$$

Assim, $F$ é localmente Lipschitz $\mathcal{H}_{0}$.

O próximo lema nos dará a existência de uma solução local fraca do problema (3.25) no intervalo fechado $[0, T]$, para algum $T>0$.

Lema 3.3. Nas mesmas condições do Teorema 3.1, suponhamos $U_{0} \in \mathcal{H}_{0}$. Então, existe um $T_{\max }>0$ tal que o problema (3.25) possui uma única solução fraca $U \in$ $C\left(\left[0, T_{\max }\right) ; \mathcal{H}_{0}\right)$ satisfazendo a fórmula de variação do parâmetros dada em (3.27). Se $U_{0} \in D(\mathcal{L})$, então $U$ é uma solução clássica.

Demonstração. Aplicaremos o Teorema 2.13. Pelo Lema 3.1, o operador $\mathcal{L}$ é gerador infitesimal de um $C_{0}$-semigrupo de contrações. Utilizando o Lema 3.2, obtemos que $F$ é localmente Lipschitz. Com isso, estamos nas hipóteses do Teorema 2.13, logo, obtemos que existe uma única solução fraca $U \in C\left(\left[0, T_{\max }\right), \mathcal{H}_{0}\right)$ do problema (3.25). Além disso, se $U_{0} \in D(\mathcal{L})$ segue do Teorema 2.15, que a solução $U$ é clássica.

\subsubsection{Prova do teorema principal}

Vamos estenter a solução fraca $U \in C\left(\left[0, T_{\max }\right) ; \mathcal{H}_{0}\right)$, dada pelo Lema 3.3, ao intervalo $[0, \infty)$. Antes, mostraremos que nossa solução é limitada no intervalo $\left[0, T_{\max }\right)$. 
Definimos o funcional de energia associado ao problema (3.6)-(3.9) como segue

$$
\begin{aligned}
E(t)= & \frac{1}{2}\left\{\left\|u_{t}(t)\right\|_{2}^{2}+\left\|\nabla u_{t}(t)\right\|_{2}^{2}+\|\Delta u(t)\|_{2}^{2}+\|\theta(t)\|_{2}^{2}+\left\|\eta^{t}\right\|_{\mu, 1}^{2}\right\} \\
& +\frac{1}{2} \mathcal{M}\left(\|\nabla u(t)\|_{2}^{2}\right)+\int_{\Omega}(\widehat{f}(u(t))-h u(t)) d x
\end{aligned}
$$

Lema 3.4. O funcional de energia definido em (3.51) satisfaz

$$
\frac{d}{d t} E(t)=-\omega\|\nabla \theta(t)\|_{2}^{2}+\frac{1}{2} \int_{0}^{\infty} \mu(s)\left\|\nabla \eta^{t}\right\|_{2}^{2} \leq 0 .
$$

Além disso, existem constantes positivas $\delta_{0}$ e $C_{M f h}$, independentes dos dados iniciais, tais que

$$
E(t) \geq \delta_{0}\left(\|\Delta u(t)\|_{2}^{2}+\left\|\nabla u_{t}\right\|_{2}^{2}+\|\theta(t)\|_{2}^{2}+\left\|\eta^{t}\right\|_{\mu, 1}^{2}\right)-C_{M f h}, \quad t \geq 0
$$

Demonstração. Pelo Teorema 2.16, se $U \in C\left(\left[0, T_{\max }\right), \mathcal{H}_{0}\right)$ é uma solução fraca do problema (3.25), então $U$ é limite uniforme de soluções clássicas do mesmo problema. Portanto, podemos trabalhar com soluções clássicas, pois o mesmo resultado seguirá, por densidade, para soluções fracas.

Seja $U_{0}=\left(u_{0}, u_{1}, \theta_{0}, \eta_{0}\right) \in D(\mathcal{L})$, então pelo Lema 3.3 , existe uma solução clássica $U(t)=\left(u(t), u_{t}(t), \theta(t), \eta^{t}\right)$ do problema (3.25) no intervalo [0,T $\left.T_{\max }\right)$. Multiplicando as equações (3.6)-(3.8) por $u_{t}, \theta$ e $\eta$, respectivamente, obtemos

$$
\begin{aligned}
& \frac{1}{2} \frac{d}{d t}\left\{\left\|u_{t}(t)\right\|_{2}^{2}\right.+\left\|\nabla u_{t}(t)\right\|_{2}^{2}+\|\Delta u(t)\|_{2}^{2}+\|\theta t(t)\|_{2}^{2}+\left\|\eta^{t}\right\|_{\mu, 1}^{2}+\mathcal{M}\left(\|\nabla u(t)\|_{2}^{2}\right) \\
&\left.+\int_{\Omega}(f(u(t))-h u(t)) d x\right\}+\nu \int_{\Omega} \Delta \theta(t) u_{t}(t) d x=0, \\
& \frac{1}{2} \frac{d}{d t}\|\theta(t)\|_{2}^{2}-\int_{0}^{\infty} \mu(s)\left(\int_{\Omega} \Delta \eta(s) \theta(t) d x\right) d s-\nu \int_{\Omega} \Delta \theta u_{t} d x+\omega\|\nabla \theta(t)\|_{2}^{2}=0
\end{aligned}
$$

$\mathrm{e}$

$$
\frac{1}{2} \frac{d}{d t}\left\|\eta^{t}\right\|_{\mu, 1}^{2}-\left(\theta(t), \eta^{t}\right)_{\mu, 1}-\frac{1}{2} \int_{0}^{\infty} \mu(s)^{\prime}\left\|\nabla \eta^{t}\right\|_{2}^{2} d s=0
$$


Combinando as igualdades (3.54)-(3.56), segue (3.52).

Observamos, a partir das hipóteses (3.11)-(3.16), que

$$
\frac{1}{2} \mathcal{M}\left(\|\nabla u(t)\|_{2}^{2}\right) \geq-\frac{\alpha}{2}\|\nabla u(t)\|_{2}^{2}-C_{M} \geq-\frac{\alpha}{2 \sqrt{\lambda_{1}}}\|\Delta u(t)\|_{2}^{2}-C_{M} .
$$

Seja $\delta_{0}>0$, a ser determinado. Pela desigualdade de Young,

$$
\int_{\Omega}(\widehat{f}(u(t))-h u(t)) d x \geq-\rho|\Omega|-\|h\|_{2}\|u(t)\|_{2} \geq-\rho|\Omega|-C_{\delta_{0} \lambda_{1}}\|h\|_{2}^{2}-\delta_{0}\|\Delta u(t)\|_{2}^{2}
$$

Escolhemos

$$
\delta_{0}=\frac{1}{2}-\frac{\alpha}{2 \sqrt{\lambda_{1}}}-\delta_{0}
$$

Então das hipótese (3.11), $\alpha<\sqrt{\lambda_{1}}$, o que mostra que $\delta_{0}>0$. Consequentemente

$$
E(t) \geq \delta_{0}\left(\|\Delta u(t)\|_{2}^{2}+\left\|\nabla u_{t}\right\|_{2}^{2}+\|\theta(t)\|_{2}^{2}+\left\|\eta^{t}\right\|_{\mu, 1}^{2}\right)-C_{M}-\rho|\Omega|-C_{\lambda_{1} \delta_{0}}\|h\|_{2}^{2} .
$$

Logo, tomando $C_{M f h}=C_{M}+\rho|\Omega|+C_{\lambda_{1} \delta_{0}}\|h\|_{2}^{2}>0$, temos (3.59).

Um corolário direto do Lema 3.4 é dado abaixo:

Corolário 3.1. Nas condições do Lema 3.4, a solução do problema (3.25) satisfaz

$$
\delta_{0}\|U(t)\|_{\mathcal{H}_{0}}^{2} \leq E(0)+C_{M f h}, \quad \forall t \in\left[0, T_{\max }\right) .
$$

Em outras palavras, segue do corolário acima que dado $U_{0} \in \mathcal{H}_{0}$, a solução $U(t)$, associada a $U_{0}$, satisfaz

$$
\lim _{t \rightarrow T_{\max }^{+}}\|U(t)\|_{\mathcal{H}_{0}}<\infty .
$$

A partir dos resultados acima, faremos a demonstração do Teorema 3.1.

Demonstração. (Teorema 3.1.) Pelo Lema 3.3, dado $U_{0} \in \mathcal{H}_{0}$, existe um $T_{\max }>0$ tal que o problema (3.25) possui única solução fraca $U \in C\left(\left[0, T_{\max }\right), \mathcal{H}_{0}\right)$. Afirmamos que $T_{\max }=\infty$. De fato, se $T_{\max }<\infty$, podemos aplicar o Teorema 2.13 para a solução $U(t)$ e dai teríamos que

$$
\lim _{t \rightarrow T_{\max }^{+}}\|U(t)\|_{\mathcal{H}_{0}}=\infty
$$


o que contrária o Corolário 3.1. Logo, $T_{\max }=\infty$. O que mostra o primeiro item do nosso teorema.

Sejam $U_{1}, U_{2} \in \mathcal{H}_{0}$ e $T>0$. Então, existem soluções fracas $U_{1}(t)$ e $U_{2}(t)$ em $[0, T]$, associadas a $U_{1}$ e $U_{2}$ respectivamente, as quais satisfazem a fórmula dada em (3.27). Assim, como essas soluções são limitadas, segue do fato que $\mathcal{L}$ é um $C_{0^{-}}$ semigrupo de contrações e que $F$ é localmente Lipschitz, que

$$
\begin{aligned}
\left\|U_{1}(t)-U_{2}(t)\right\|_{\mathcal{H}_{0}} & \leq\left\|e^{\mathcal{L}}\left(U_{1}-U_{2}\right)\right\|_{\mathcal{H}_{0}}+\int_{0}^{t}\left\|e^{\mathcal{L}(t-s)}\left(F\left(U_{1}(s)\right)-F\left(U_{2}(s)\right)\right)\right\|_{\mathcal{H}_{0}} d s \\
& \left.\leq\left\|U_{1}-U_{2}\right\|_{\mathcal{H}_{0}}+L_{0 M f} \int_{0}^{t} \| U_{1}(s)\right)-U_{2}(s) \|_{\mathcal{H}_{0}} d s
\end{aligned}
$$

Finalmente, o resultado decorre da desigualdade de Gronwall com $\sigma=L_{0 M f}$. Isso demonstra o segundo item do nosso teorema.

Observação 3.1. Se $U(t)=\left(u(t), v(t), \theta(t), \eta^{t}\right)$ é solução do problema (3.25) com dado inicial $U_{0}=\left(u_{0}, u_{1}, \theta_{0}, \eta_{0}\right) \in \mathcal{H}_{0}$. Então a terna $z(t)=\left(u(t), \theta(t), \eta^{t}\right)$ satisfaz as equações do problema (3.6)-(3.9). Mais ainda, como $U \in C\left([0, \infty) ; \mathcal{H}_{0}\right)$, temos

$$
u \in C\left([0, \infty) ; V_{2}\right) \cap C^{1}\left([0, \infty) ; V_{1}\right), \quad \theta \in C\left([0, \infty) ; V_{0}\right), \quad \eta^{t} \in C\left([0, \infty) ; \mathcal{M}_{1}\right)
$$

Por outro lado, segue do Lema 3.4 que

$$
\frac{d}{d t} E(t)=-\omega\|\nabla \theta(t)\|_{2}^{2}+\frac{1}{2} \int_{0}^{\infty} \mu^{\prime}(s)\left\|\eta^{t}(s)\right\|_{V_{1}}^{2} d s .
$$

Na demonstração do Teorema 3.1, provamos que

$$
\delta_{0}\|U(t)\|_{\mathcal{H}_{0}}^{2} \leq E(0)+C_{M f h}, \quad t \in[0, \infty)
$$

em que o lado direito da desigualdade não depende do tempo t. Logo, obtemos integrando de 0 atét, que

$$
\delta_{0}\|U(t)\|_{\mathcal{H}_{0}}^{2} \leq E(t)+C_{M f h}+\omega \int_{0}^{t}\|\nabla \theta(\tau)\|_{2}^{2} d \tau+\frac{\gamma_{0}}{2} \int_{0}^{t}\left\|\eta^{\tau}\right\|_{\mu, 1}^{2} d \tau \leq E(0)+C_{M f h}
$$


Portanto, $U(t) \in L^{\infty}\left([0, \infty) ; \mathcal{H}_{0}\right)$ e temos a seguinte regularidade

$$
\begin{array}{r}
u \in L^{\infty}\left(0, \infty ; V_{2}\right), u_{t} \in L^{\infty}\left(0, \infty ; V_{1}\right), \quad \theta \in L^{\infty}\left(0, \infty ; V_{0}\right) \cap L^{2}\left(0, \infty ; V_{1}\right), \\
\eta^{t} \in L^{\infty}\left(0, \infty ; \mathcal{M}_{1}\right) \cap L^{2}\left(0, \infty ; \mathcal{M}_{1}\right) .
\end{array}
$$

O próximo resultado estabelece uma fórmula de representação para a variável $\eta^{t}$. Adotaremos o mesmo procedimento utilizado em Grasselli \& Pata [22] e Potomkim[36].

Proposição 3.2. Seja $U(t)=\left(u(t), v(t), \theta(t), \eta^{t}\right)$ uma solução fraca do problema (3.25) com dado inicial $U_{0}=\left(u_{0}, v_{0}, \theta_{0}, \eta_{0}\right) \in \mathcal{H}_{0}$. Então

$$
\eta^{t}(s)=\left\{\begin{array}{l}
\int_{0}^{s} \theta(t-\tau) d \tau, \quad t \geq s>0, \\
\eta_{0}(s-t)+\int_{0}^{t} \theta(t-d \tau) d \tau, \quad s>t .
\end{array}\right.
$$

Demonstração. Seja $\left\{U_{0 n} ; U_{0 n} \in D(\mathcal{L})\right\}$, tal que $U_{0 n} \stackrel{n \rightarrow \infty}{\longrightarrow} U_{0} \in \mathcal{H}_{0}$. Segue do Teorema 2.16, que, para cada $T>0$ arbitrário, existe uma sequência $\left\{U_{n}(t)\right\}_{n=0}^{\infty}$ de soluções clássicas associada a $\left\{U_{0 n} ; U_{0 n} \in D(\mathcal{L})\right\}$ tal que

$$
U_{n}(t) \stackrel{n \rightarrow \infty}{\longrightarrow} U(t)
$$

uniformemente em $[0, T]$, em que $U(t)$ é a solução do problema (3.25) associada ao dado $U_{0} \in \mathcal{H}_{0}$. Para cada $n \in \mathbb{N}, U_{n}(t)=\left(u_{n}(t), u_{n t}(t), \theta_{n}(t), \eta_{n}^{t}\right)$. Então, sendo $U_{n}(t)$ solução do problema(3.25), $\eta_{n}^{t}$ satisfaz a equação (3.8), isto é,

$$
\partial_{t} \eta_{n}^{t}(s)=-\partial_{s} \eta_{n}^{t}(s)+\theta_{n}(t)
$$

Seja $y=t-s$. Fazendo a substituição na equação acima, obtemos

$$
\partial_{t} \eta_{n}^{t}(t-y)=-\partial_{y} \eta_{n}^{t}(t-y)+\theta_{n}(t)
$$

Por outro lado, aplicando a regra da cadeia a $\eta^{t}(t-y)$, observamos que

$$
\frac{d}{d t} \eta_{n}^{t}(t-y)=\partial_{t} \eta_{n}^{t}(t-y)-\partial_{y} \eta_{n}^{t}(t-y)
$$

Portanto, obtemos a seguinte equação diferencial 


$$
\frac{d}{d t} \eta_{n}^{t}(t-y)=\theta_{n}(t)
$$

Lembramos que $\eta_{n}^{t}(0)=0$ e $t=s+y$. Para $t \geq s>0$, integrando (3.67) de $y$ até $t$, temos

$$
\eta_{n}^{t}(t-y)=\int_{y}^{t} \theta_{n}(\tau) d \tau .
$$

Por outro lado, por mudança de variável, que

$$
\eta_{n}^{t}(s)=\int_{0}^{s} \theta_{n}(t-\xi) d \xi
$$

Agora, para $s>t$, integrando (3.67) de 0 até $t$, chegamos a

$$
\eta_{n}^{t}(t-y)-\eta_{0 n}(-y)=\int_{0}^{t} \theta_{n}(\tau) d \tau
$$

Observamos agora que $-y=s-t$. Utilizando mudança de variáveis, temos

$$
\eta_{n}^{t}(s)=\eta_{0 n}(s-t)+\int_{0}^{t} \theta_{n}(t-\xi) d \xi
$$

Seja

$$
\varphi_{n}^{t}(s)=\left\{\begin{array}{l}
\int_{0}^{s} \theta_{n}(t-\xi) d \xi, t \geq s>0, \\
\eta_{0 n}(s-t)+\int_{0}^{t} \theta_{n}(t-\xi) d \xi, \quad s>t
\end{array}\right.
$$

e

$$
\varphi^{t}(s)=\left\{\begin{array}{l}
\int_{0}^{s} \theta(t-\xi) d \xi, t \geq s>0, \\
\eta_{0}(s-t)+\int_{0}^{t} \theta(t-\xi) d \xi, \quad s>t .
\end{array}\right.
$$

Por construção, $\varphi_{n}^{t}(s)=\eta_{n}^{t}(s)$. Mostraremos que $\varphi^{t}(s)=\eta^{t}(s)$. De fato, como

$$
\eta_{n}^{t}(s) \rightarrow \eta^{t}(s)
$$

em $\mathcal{M}_{1}$ uniformemente em $[0, T]$, é suficiente mostrar que

$$
\varphi_{n}^{t}(s) \rightarrow \varphi^{t}(s)
$$


em $\mathcal{M}_{1}$ para cada $t \in[0, t]$

$$
\begin{aligned}
\left\|\varphi_{n}^{t}-\varphi^{t}\right\|_{\mu, 1}^{2}= & \int_{0}^{\infty} \mu(s)\left\|\varphi_{n}^{t}(s)-\varphi^{t}(s)\right\|_{V_{1}}^{2} d s \leq \int_{0}^{t} \mu(s)\left\|\int_{0}^{s} \theta_{n}(t-\xi)-\theta(t-\xi) d \xi\right\|_{V_{1}}^{2} d s \\
& +\int_{t}^{\infty} \mu(s)\left\|\eta_{0 n}(s-t)-\eta_{0}(s-t)\right\|_{V_{1}}^{2} d s \\
& +\int_{t}^{\infty} \mu(s)\left\|\int_{0}^{t} \theta_{n}(t-\xi)-\theta(t-\xi) d \xi\right\|_{V_{1}}^{2} d s
\end{aligned}
$$

Cada termo acima converge pontualmente no intervalo $[0, T]$, pois

$$
\begin{aligned}
& \int_{0}^{t} \mu(s) \sup _{0 \leq s \leq t}\left\|\int_{0}^{s} \theta_{n}(t-\xi)-\theta(t-\xi) d \xi\right\|_{V_{1}}^{2} d s \leq k_{1}\left\|\int_{0}^{t} \theta_{n}(t-\xi)-\theta(t-\xi) d \xi\right\|_{V_{1}}^{2} ; \\
& \int_{t}^{\infty} \mu(s)\left\|\int_{0}^{t} \theta_{n}(t-\xi)-\theta(t-\xi) d \xi\right\|_{V_{1}}^{2} d s \leq k_{1}\left\|\int_{0}^{t} \theta_{n}(t-\xi)-\theta(t-\xi) d \xi\right\|_{V_{1}}^{2} ; \\
& \int_{t}^{\infty} \mu(s)\left\|\eta_{0 n}(s-t)-\eta_{0}(s-t)\right\|_{V_{1}}^{2} d s \leq\left\|\eta_{0 n}(s-t)-\eta_{0}(s-t)\right\|_{\mu, 1}^{2} .
\end{aligned}
$$

Pela convergência uniforme, dado $\epsilon>0$, existem $n_{1}, n_{2} \in \mathbb{N}$ de modo que, para todo $n \geq \max \left\{n_{1}, n_{2}\right\}$ temos

$$
\left\|\theta_{n}(t-\xi)-\theta(t-\xi)\right\|_{V_{1}} \leq \sqrt{\frac{\epsilon}{2 k_{1}}}
$$

e

$$
\left\|\eta_{0 n}(s-t)-\eta(s-t)\right\|_{\mu, 1} \leq \sqrt{\epsilon}
$$

Logo,

$$
\left\|\varphi_{n}^{t}-\varphi^{t}\right\|_{\mu, 1}^{2} \rightarrow 0
$$

para todo $t \in[0, T]$. Assim, obtemos a convergência pontual em $t$. Lembramos que $\varphi_{n}^{t}(s)=\eta_{n}^{t}(s)$ para todo $s \geq 0$ e $t \in[0, T]$. Decorre, portanto, da convergência uniforme em $(3.71$,$) que \varphi^{t}(s)=\eta^{t}(s)$ para todo $s \geq 0$ e $t \in[0, T]$. 



\section{Capítulo 4}

\section{Atratores de dimensão finita}

Neste capítulo, estudaremos a existência do Atrator Global de dimensão fractal finita. Apresentaremos algumas definições e resultados referentes à teoria de atratores mencionados no primeiro capítulo. Para maiores detalhes sobre o assunto, consultar Babim \& Vishik [2], Hale [26], Ladyzhenskaya [29] e Temam [39]. Adotaremos o método apresntado no livro de Chueshov \& Lasiecka [10, 11].

Observação 4.1. Lembramos que $\mathcal{H}_{0}=V_{2} \times V_{1} \times V_{0} \times \mathcal{M}_{1}$. Segue do Teorema 3.1 que o operador

$$
S(t): \mathcal{H}_{0} \rightarrow \mathcal{H}_{0}, \quad S(t)\left(u_{0}, u_{1}, \theta_{0}, \eta_{0}\right)=\left(u(t), u_{t}(t), \theta(t), \eta^{t}\right)
$$

em que $U(t)=\left(u(t), u_{t}(t), \theta(t), \eta^{t}\right)$ é a única solução do problema (3.6)-(3.8) com condição inicial $U_{0}=\left(u_{0}, u_{1}, \theta_{0}, \eta_{0}\right)$, satisfaz as propriedades de semigrupo. Mais precisamente,

$$
\begin{aligned}
& \left.S(0)=I d: \mathcal{H}_{0} \rightarrow \mathcal{H}_{0} \quad \text { (Operador Identidade }\right), \\
& S(t+s)=S(t) \circ S(s), \quad s, t \geq 0 .
\end{aligned}
$$

$A$ dependência contínua em relação aos dados iniciais nos garante que $S(t): \mathcal{H}_{0} \rightarrow \mathcal{H}_{0}$ é fortemente contínuo, para $t$ fixo. Além disso, segue de $U \in C\left([0, T], \mathcal{H}_{0}\right)$ que a aplicação

$$
[0, \infty) \times \mathcal{H}_{0} \ni(t, x) \rightarrow S(t) x \in \mathcal{H}_{0}
$$

é contínua para cada $x$ fixo. Portanto, o problema (3.6)-(3.8) produz um sistema dinâmico não-linear $\left(\mathcal{H}_{0}, S(t)\right)$. 
Teorema 4.1. O sistema dinâmico $\left(\mathcal{H}_{0}, S(t)\right)$ dado pelo Teorema 3.1 é gradiente $e$ possui um atrator global $\mathcal{A}$ de dimensão fractal finita. Além disso,

$$
\mathcal{A}=\mathcal{W}^{u}(\mathcal{N})
$$

em que $\mathcal{N}$ é o conjunto das soluções estacionárias do problema (3.25).

Observação 4.2. O atrator $\mathcal{A}$, dado pelo Teorema 4.1 também pode ser visto como o conjunto de todas as trajetórias completas $\gamma=\{u(t) ; t \in \mathbb{R}\}$ tais que

$$
\lim _{t \rightarrow-\infty} \operatorname{dist}_{X}(u(t), \mathcal{N})=0 \text { e } \lim _{t \rightarrow \infty} \operatorname{dist}_{X}(u(t), \mathcal{N})=0 .
$$

Esse fato segue do Teorema 2.20.

Para obter a existência do atrator global, mostraremos que nosso sistema dinâmico é assintoticamente suave, gradiente, cujo o funcional de Lyapunov $\Phi$ satisfaz as hipóteses do Corolário 2.2. Através do método de perturbação de energia, obteremos uma desigualdade conhecida como desigualdade de estabilização. Com isso, demonstraremos que $\left(\mathcal{H}_{0}, S(t)\right)$ é quase-estável, obtendo, assim, a propriedade de ser assintoticamente suave. Por fim, aplicando o Corolário 2.2 e obtemos a existência do atrator. A dimensão finita seguirá do Teorema 2.18. Além disso, com alguns ajustes nessa desigualdade e a propriedade de Hölder contínua para o semigrupo $S(t)$, poderemos demonstrar a existência de um atrator exponencial generalizado.

\subsection{Sistema dinâmico gradiente}

Nessa seção mostraremos que nosso sistema dinâmico satisfaz as hipóteses do Corolário 2.2.

\subsubsection{Conjunto das soluções estacionárias}

Seja $\mathcal{N}$ o conjunto das soluções estacionários do problema (3.25), ou seja,

$$
\mathcal{N}=\left\{U \in \mathcal{H}_{0} ; S(t) U=U, \forall t \geq 0\right\}
$$


Notamos que, se $U_{0} \in \mathcal{H}_{0}$ é uma solução fraca estacionária do problema (3.25), então

$$
U_{0}=e^{t \mathcal{L}} U_{0}+\int_{0}^{t} e^{(t-s) \mathcal{L}} F\left(U_{0}\right) d s
$$

o que nos leva a

$$
-\left(\frac{e^{t \mathcal{L}}-I d}{t}\right) U_{0}=\frac{1}{t} \int_{0}^{t} e^{(t-s) \mathcal{L}} F\left(U_{0}\right) d s=\frac{1}{t} \int_{0}^{t} e^{\tau \mathcal{L}} F\left(U_{0}\right) d \tau .
$$

O lado esquerdo dessa igualdade é igual a $-\mathcal{L} U_{0}$. Por outro lado, nosso semigrupo é fortemente contínuo, isto é,

$$
\lim _{t \rightarrow t_{0}} e^{t \mathcal{L}} U=e^{t_{0} \mathcal{L}} U
$$

para todo $U \in \mathcal{H}_{0}$. Logo,

$$
\left\|\frac{1}{t} \int_{0}^{t} e^{\tau \mathcal{L}} F\left(U_{0}\right) d s-F\left(U_{0}\right)\right\|_{\mathcal{H}_{0}} \leq \frac{1}{t} \int_{0}^{t}\left\|e^{\tau \mathcal{L}} F\left(U_{0}\right) d s-e^{0 \mathcal{L}} F\left(U_{0}\right)\right\|_{\mathcal{H}_{0}} d s \rightarrow 0,
$$

quando $t \rightarrow 0$. Assim, o lado direito de (4.3) é, exatamente, $F\left(U_{0}\right)$. Portanto, chegamos a seguinte igualdade

$$
\mathcal{L}\left(U_{0}\right)+F\left(U_{0}\right)=0
$$

Logo, segue da igualdade acima que o conjunto $\mathcal{N}$ é dado pela seguinte proposição:

Proposição 4.1. O conjunto $\mathcal{N}$ pode ser escrito da seguinte forma

$$
\mathcal{N}=\left\{U=\left(u_{0}, 0,0,0\right) ; \Delta^{2} u_{0}-M\left(\|\nabla u\|_{2}^{2}\right) \Delta u_{0}+f\left(u_{0}\right)=h\right\}, \quad u \in V_{2} .
$$

Além disso, $\mathcal{N} \subset \mathcal{D}(\mathcal{L})$.

Lema 4.1. O sistema dinâmico $\left(\mathcal{H}_{0}, S(t)\right)$, produzido pelo problema (3.6)-(3.8), é gradiente.

Demonstração. Seja $U(t)=\left(u(t), u_{t}(t), \theta(t), \eta^{t}\right)$ a solução do problema (3.25) dada pelo Teorema 3.1. Defina o funcional $\Phi(U(t))=E(t)$, em que $E(t)$ é a energia associada ao problema (3.6)-(3.8) definida em (3.51). Como a energia é decrescente, $\Phi$ é não 
crescente sobre qualquer solução $U(t)$ do problema (3.25). Pelo Teorema 3.1, para cada $U_{0}=\left(u_{0}, u_{1}, \theta_{0}, \eta_{0}\right) \in D(\mathcal{L})$, existe uma única solução clássica $U(t)=\left(u(t), u_{t}(t), \theta(t), \eta^{t}\right)$. Aplicando o Lema 3.4, observamos que

$$
\Phi^{\prime}\left(S(t) U_{0}\right)=-\omega\|\nabla \theta(t)\|_{2}^{2}+\frac{1}{2} \int_{0}^{\infty} \mu^{\prime}(s)\left\|\nabla \eta^{t}(s)\right\|_{2}^{2} d s,
$$

sobre $U(t)=S(t) U_{0}$. Se $U(t)$ é uma solução estacionária, $\Phi^{\prime}\left(S(t) U_{0}\right)=0$, para todo $t \geq 0$. Suponhamos que $\Phi\left(S(t) U_{0}\right)=S\left(U_{0}\right)$. Então, a partir de (4.6), obtemos

$$
\omega\|\nabla \theta(t)\|_{2}^{2}-\frac{1}{2} \int_{0}^{\infty} \mu^{\prime}(s)\left\|\nabla \eta^{t}(s)\right\|_{2}^{2} d s=0 .
$$

Utilizando o Lema 3.4 e Proposição 3.1, sabemos que os dois termos da expressão do lado esquerdo possuem o mesmo sinal. Logo, devemos ter

$$
\|\nabla \theta(t)\|_{2}=0
$$

e

$$
\int_{0}^{\infty} \mu^{\prime}(s)\left\|\nabla \eta^{t}(s)\right\|_{2}^{2} d s=0, \quad \forall t \geq 0
$$

Seja

$$
\sigma_{\infty}=\sup \{s ; \mu(s)>0\}
$$

Então, $\mu^{\prime}(s) \leq \gamma_{0} \mu(s)<0$, para $s \in\left(0, \sigma_{\infty}\right)$. Assim, temos

$$
\eta^{t}(x, s)=0, q . s, \quad \mathrm{em}(x, s) \in \Omega \times\left(0, \sigma_{\infty}\right), \quad t \geq 0 .
$$

Além disso, obtemos

$$
\int_{0}^{\infty} \mu(s) \Delta \eta^{t}(s) d s=\int_{0}^{\sigma_{\infty}} \mu(s) \Delta \eta^{t}(s) d s+\int_{\sigma_{\infty}}^{\infty} \mu(s) \Delta \eta^{t}(s) d s=0 .
$$

Como $\theta$ não depende de $s$, segue da equação (3.8) que $\theta(t) \equiv 0$. Utilizando a equação (3.7), obtemos $\Delta u_{t}(t)=0$, para todo $t \geq 0$, aplicando a desigualdade de Poincaré, $u_{t} \equiv 0$, o que nos mostra que $u \equiv u_{0}$. Logo, $U(t)=\left(u_{0}, 0,0,0\right)$. Ademais, segue da equação (3.25) que

$$
\Delta^{2} u_{0}-M\left(\left\|\nabla u_{0}\right\|_{2}^{2}\right) \Delta u_{0}+f\left(u_{0}\right)=h
$$

Portanto, $U(t) \in \mathcal{N}$. 
Lema 4.2. O conjunto $\mathcal{N}$ das soluções estacionárias do problema (3.25) é limitado em $\mathcal{H}_{0}$.

Demonstração. Seja $U \in \mathcal{N}$, então, como $\mathcal{N} \subset D(\mathcal{L})$, temos

$$
\Delta^{2} u_{0}-M\left(\left\|\nabla u_{0}\right\|_{2}^{2}\right) \Delta u_{0}+f\left(u_{0}\right)=h, u_{0} \in V_{3}
$$

Então, da igualdade acima, temos

$$
\left\|\Delta u_{0}\right\|_{2}^{2}+M\left(\left\|\nabla u_{0}\right\|_{2}^{2}\right)\left\|\nabla u_{0}\right\|_{2}^{2}+\int_{\Omega} f\left(u_{0}\right) u_{0} d x=\int_{\Omega} h u_{0} d x .
$$

Por outro lado, a partir das hipóteses (3.11)-(3.16), observamos que

$$
\int_{\Omega} f\left(u_{0}\right) u_{0} d x \geq-\rho|\Omega|
$$

e

$$
M\left(\left\|\nabla u_{0}\right\|_{2}^{2}\right)\left\|\nabla u_{0}\right\|_{2}^{2} \geq-\frac{\alpha}{\lambda_{1}}\left\|\Delta u_{0}\right\|_{2}^{2}-\frac{3}{2} c_{M} .
$$

Dado $\epsilon>0$, temos da desigualdade de Young que

$$
\int_{\Omega} h u_{0} d x \leq \epsilon\left\|\Delta u_{0}\right\|_{2}^{2}+\frac{1}{4 \epsilon \lambda_{1}^{2}}\|h\|_{2}^{2}
$$

Combinando as desigualdades acima, chegamos a seguinte desigualdade

$$
\left(1-\frac{\alpha}{\lambda_{1}}-\epsilon\right)\left\|\Delta u_{0}\right\|_{2}^{2} \leq \rho|\Omega|+\frac{3}{2} c_{M}+\frac{1}{4 \epsilon \lambda_{1}^{2}} .
$$

Logo, fixando $0<\epsilon<1-\frac{\alpha}{\lambda_{1}}$ obtemos que $\mathcal{N}$ é limitado.

\subsection{Sistema quase-estável}

Mostraremos nessa seção que o sistema dinâmico gerado pelo Teorema 3.1 é quase-estável. 


\subsubsection{Desigualdade de estabilização}

Lema 4.3. Nas hipóteses do Teorema 4.1, sejam $B \subset \mathcal{H}_{0}$ um subconjunto limitado, $z^{1}(t)=\left(u(t), u_{t}(t), \theta(t), \eta^{t}\right)$ e $z^{2}(t)=\left(v(t), v_{t}(t), \widetilde{\theta}(t), \widetilde{\eta}^{t}\right)$ duas soluções do problema (3.6)-(3.8) com dados iniciais $z^{1}(0)=\left(u_{0}, u_{1}, \theta_{0}, \eta_{0}\right)$ e $z^{2}(0)=\left(v_{0}, v_{1}, \widetilde{\theta}_{0}, \widetilde{\eta}_{0}\right)$ em $B$, respectivamente. Então, existem constantes positivas $\sigma$ e $C_{B}$, dependendo de $B$, tais que

$$
\left\|z^{1}(t)-z^{2}(t)\right\|_{\mathcal{H}_{0}}^{2} \leq C e^{-\sigma t}\left\|z^{1}(0)-z^{2}(0)\right\|_{\mathcal{H}_{0}}^{2}+K_{B} \int_{0}^{t} e^{-\sigma(t-s)}\|\nabla w(s)\|_{2}^{2} d s,
$$

em que $w=u-v$.

Observação 4.3. Antes do início da prova do Lema 4.3, apresentamos a seguinte desigualdade que será útil na demonstração. Como $\mu^{\prime}(s)+\gamma_{0} \mu(s) \leq 0$, temos

$$
\gamma_{0} \int_{0}^{\infty} \mu(s)\left\|\nabla \eta^{t}(s)\right\|_{2}^{2} d s \leq-\int_{0}^{\infty} \mu^{\prime}(s)\left\|\nabla \eta^{t}(s)\right\|_{2}^{2} d s .
$$

Demonstração. ( Lema 4.3)

Denotamos $\tau=\theta-\widetilde{\theta}$ e $\xi=\eta-\widetilde{\eta}$. Então $\widetilde{z}(t)=\left(w(t), w_{t}(t), \tau(t), \xi^{t}\right)$ é uma solução fraca do sistema de equações

$$
\begin{aligned}
& w_{t t}+\Delta^{2} w-\Delta w_{t t}+\nu \Delta \tau=M\left(\|\nabla u\|_{2}^{2}\right) \Delta u-M\left(\|\nabla v\|_{2}^{2}\right) \Delta u+f(v)-f(u), \\
& \tau_{t}-\omega \Delta \tau+\int_{0}^{\infty} \mu(s) \Delta \xi^{t}(s) d s+\nu \Delta w_{t}=0, \\
& \xi_{t}^{t}=-\xi_{s}^{t}+\tau
\end{aligned}
$$

com condições iniciais

$$
w_{0}=u_{0}-v_{0}, w_{1}=u_{1}-v_{1}, \tau_{0}=\theta_{0}-\widetilde{\theta}_{0} \text { e } \xi_{0}=\eta_{0}-\widetilde{\eta}_{0}
$$

e condições de fronteira nulas. O funcional de energia associado ao problema (3.18)(4.15) é dado por

$$
F(t)=\frac{1}{2}\left\{\left\|w_{t}(t)\right\|_{2}^{2}+\left\|\nabla w_{t}(t)\right\|_{2}^{2}+\|\Delta w(t)\|_{2}^{2}+\|\tau(t)\|_{2}^{2}+\left\|\xi^{t}\right\|_{2}^{2}\right\}
$$


Etapa1 Dado $\delta_{0}>0$, existe $C_{\delta_{0}}>0$, dependendo de $B$, de modo que

$$
F^{\prime}(t) \leq-\omega\|\nabla \tau(t)\|_{2}^{2}+\frac{1}{2} \int_{0}^{\infty} \mu^{\prime}(s)\left\|\nabla \xi^{t}(s)\right\|_{2}^{2} d s+\delta_{0}\left\|\nabla w_{t}(t)\right\|+C_{\delta_{0}}\|\nabla w(t)\|_{2}^{2}
$$

Com efeito, multiplicando por $w_{t}, \tau$ e $\xi$ as equações $(4.13),(4.14)$ e (4.15), respectivamente, temos

$$
F^{\prime}(t) \leq-\omega\|\nabla \tau(t)\|_{2}^{2}+\frac{1}{2} \int_{0}^{\infty} \mu^{\prime}(s)\left\|\nabla \xi^{t}(s)\right\|_{2}^{2} d s+M f
$$

em que

$$
\begin{aligned}
M f & =\int_{\Omega}\left(M\left(\|\nabla u(t)\|_{2}^{2}\right) \Delta u(t)-M\left(\|\nabla v(t)\|_{2}^{2}\right) \Delta v(t)\right) w_{t}(t) d x \\
& -\int_{\Omega}(f(u(t))-f(v(t))) w_{t}(t) d x .
\end{aligned}
$$

Mostraremos que existe uma constante positiva $C_{M f}>0$, que depende de $B$, tal que

$$
M f \leq C_{M f}\|\nabla w(t)\|_{2}^{2}\left\|\nabla w_{t}(t)\right\|_{2}^{2}
$$

Com efeito, seja $\triangle M=M\left(\|\nabla u(t)\|^{2}\right)-M\left(\|\nabla v(t)\|^{2}\right)$. Então, $M\left(\|\nabla u(t)\|_{2}^{2}\right) \Delta u(t)-M\left(\|\nabla v(t)\|_{2}^{2}\right) \Delta v(t)=\triangle M \Delta u(t)+M\left(\|\nabla v(t)\|_{2}^{2}\right)(\Delta u(t)-\Delta v(t))$.

Pelo teorema do valor médio, existe uma constante $C_{M}>0$ tal que

$$
\triangle M=M\left(\|\nabla u(t)\|_{2}^{2}\right)-M\left(\|\nabla v(t)\|_{2}^{2}\right) \leq C_{M}\|\nabla u(t)-\nabla v(t)\|_{2},
$$

em que $C_{M}>0$ é uma constante que depende do limitado $B$. Logo, existe uma constante $\mathcal{C}_{M}>0$, que depende de $B$, de forma que

$$
\begin{aligned}
& \int_{\Omega}\left(M\left(\|\nabla u(t)\|_{2}^{2}\right) \Delta u(t)-M\left(\|\nabla v(t)\|_{2}^{2}\right) \Delta v(t)\right) w_{t}(t) d x=\Delta M \int_{\Omega} \nabla u(t) \nabla w_{t}(t) d x \\
& +\int_{\Omega} M\left(\|\nabla v(t)\|_{2}^{2}\right)(\Delta u(t)-\Delta v(t)) w_{t}(t) d x \leq \mathcal{C}_{M}\|\nabla w(t)\|_{2}\left\|\nabla w_{t}(t)\right\|_{2} .
\end{aligned}
$$

Analogamente, mostramos que existe $C_{f}>0$, dependendo do subconjunto limitado $B$, tal que 


$$
\int_{\Omega}(f(u(t))-f(v(t))) w_{t} \leq C_{f}\|\nabla w(t)\|_{2}\left\|\nabla w_{t}(t)\right\|_{2} .
$$

Portanto, existe uma constante $C_{M f}>0$, dependente do subconjunto $B$, tal que

$$
M f \leq C_{M f}\|\nabla w(t)\|_{2}\left\|\nabla w_{t}(t)\right\|_{2} .
$$

Aplicando a desigualdade de Young para $\delta_{0}>0$ ao termo $\|\nabla w(t)\|_{2}\left\|\nabla w_{t}(t)\right\|_{2}$, obtemos a desigualdade (4.18). Seja $p(t)$ a solução do P.V.I

$$
\left\{\begin{array}{c}
-\Delta p(t)=\tau(t), \Omega, \\
p(0)=0, \partial \Omega .
\end{array}\right.
$$

Então $p(t)=\Delta^{-1} \tau(t)$, usando Poincaré obtemos que

$$
\left\|\Delta^{-1} \tau(t)\right\|_{2}=\|p(t)\|_{2} \leq \frac{1}{\lambda_{1}}\|\tau(t)\|_{2} .
$$

Definimos os seguintes funcionais

$$
\begin{aligned}
& \psi(t)=\int_{\Omega}\left(w_{t}(t)-\Delta w_{t}(t)\right) w(t) d x, \\
& \phi(t)=\int_{\Omega} w_{t}(t) \tau(t) d x-\int_{\omega} w_{t}(t) \Delta^{-1} \tau(t) d x, \\
& \chi(t)=-\int_{0}^{\infty} \mu(s)\left(\int_{\Omega} \tau(t) \xi^{t}(s)\right) d s,
\end{aligned}
$$

Definimos o funcional de Lyapunov como segue

$$
\mathcal{J}(t)=N F(t)+\epsilon_{1} \epsilon_{2} \psi(t)+\epsilon_{2} \phi(t)+\chi(t)
$$

em que $\epsilon_{1}, \epsilon_{2} \in(0,1)$ e $N \in \mathbb{N}$ serão fixados mais adiante.

Etapa 2 Existem constantes positivas $\beta_{1}$ e $\beta_{2}$ tais que

$$
\beta_{1} F(t) \leq \mathcal{J}(t) \leq \beta_{2} F(t)
$$


De fato, observamos que

$$
\begin{aligned}
& |\psi(t)| \leq\left\|w_{t}(t)\right\|_{2}^{2}+C_{s}\|\Delta w\|_{2}^{2}, \\
& |\chi(t)| \leq \frac{k_{0}}{2}\|\tau(t)\|_{2}^{2}+\frac{1}{2}\left\|\xi^{t}\right\|_{\mu, 1}^{2}
\end{aligned}
$$

e

$$
|\phi(t)| \leq\|\tau(t)\|_{2}^{2}+C_{s}\left\|w_{t}(t)\right\|_{2}^{2}
$$

Segue do Lema 3.4, Capítulo 3, que

$$
\begin{aligned}
|\psi(t)+\phi(t)+\chi(t)| & \leq \beta_{0} \delta_{0}\left(\left\|w_{t}(t)\right\|_{2}^{2}+\left\|\nabla w_{t}(t)\right\|_{2}^{2}+\|\Delta w(t)\|_{2}^{2}+\|\tau(t)\|_{2}^{2}+\left\|\xi^{t}\right\|_{2}^{2}\right) \\
& \leq \beta_{0} F(t) .
\end{aligned}
$$

Por fim, seja $N>\beta_{0}$ e $\beta_{1}=N-\beta_{0}$ e $\beta_{2}=N+\beta_{0}$. Obtemos, desse modo, as desigualdades em (4.27).

Etapa 3 Existe uma constante $C_{1}>0$, dependendo de $B$, tal que

$$
\begin{aligned}
\psi^{\prime}(t) & \leq-F(t)+\frac{3}{2}\left\|w_{t}\right\|_{2}^{2}+\frac{3}{2}\left\|\nabla w_{t}(t)\right\|_{2}^{2}-\frac{1}{4}\|\Delta w(t)\|_{2}^{2} \\
& +C_{1}\|\tau(t)\|_{2}^{2}+C_{1}\|\nabla w(t)\|_{2}^{2}-C_{1} \int_{0}^{\infty} \mu^{\prime}(s)\left\|\nabla \xi^{t}(s)\right\|_{2}^{2} d s .
\end{aligned}
$$

Com efeito, temos

$$
\psi^{\prime}(t)=\int_{\Omega}\left(w_{t t}(t)-\Delta w_{t t}(t)\right) w(t) d x+\left\|w_{t}(t)\right\|_{2}^{2}+\left\|\nabla w_{t}(t)\right\|_{2}^{2}
$$

Por outro lado, a partir da equação (4.13) observamos que

$$
\begin{aligned}
\int_{\Omega}\left(w_{t t}(t)-\Delta w_{t t}(t)\right) w(t) d x= & -\|\Delta w(t)\|_{2}^{2}-\nu \int_{\Omega} \Delta \tau(t) w(t) d x \\
& +\int_{\Omega}(f(v(t))-f(u(t))) w(t) d x \\
& +\int_{\Omega}\left(M\left(\|\nabla u(t)\|_{2}^{2}\right) \Delta u(t)-M\left(\|\nabla v(t)\|_{2}^{2}\right) \Delta v(t)\right) w(t) d x \\
\leq & -\|\Delta w(t)\|_{2}^{2}+\nu\|\tau(t)\|_{2}\|\Delta w(t)\|_{2}+C_{M f}\|\nabla w(t)\|_{2}^{2} .
\end{aligned}
$$


Adicionando e subtraindo $F(t)$ em $\psi^{\prime}(t)$ obtemos que

$$
\begin{aligned}
\psi^{\prime}(t) \leq-F(t) & +\frac{3}{2}\left\|w_{t}(t)\right\|_{2}^{2}+\frac{3}{2}\left\|\nabla w_{t}(t)\right\|_{2}^{2}+\frac{1}{2}\|\tau(t)\|_{2}^{2}+\frac{1}{2}\left\|\xi^{t}\right\|_{\mu, 1}^{2}-\frac{1}{2}\|\Delta w(t)\|_{2}^{2} \\
& +\nu\|\tau(t)\|_{2}\|\Delta w(t)\|_{2}+C_{M f}\|\nabla w(t)\|_{2}^{2}
\end{aligned}
$$

Dado $\delta>0$, aplicando a desigualdade de Young teremos

$$
\nu\|\tau(t)\|_{2}\|\Delta w(t)\|_{2} \leq \delta\|\Delta w(t)\|_{2}^{2}+C_{\delta \nu}\|\tau(t)\|_{2}^{2}
$$

Escolhendo $\delta=\frac{1}{4}$, combinando as desigualdades acima e usando a observação 4.3, temos

$$
\begin{aligned}
\psi^{\prime}(t) \leq-F(t) & +\frac{3}{2}\left\|w_{t}(t)\right\|_{2}^{2}+\frac{3}{2}\left\|\nabla w_{t}(t)\right\|_{2}^{2}+\frac{1}{2}\|\tau(t)\|_{2}^{2}-\frac{1}{4}\|\Delta w(t)\|_{2}^{2} \\
& +C_{\nu}\|\tau(t)\|_{2}^{2}-\frac{1}{2 \gamma_{0}} \int_{0}^{\infty} \mu^{\prime}(s)\left\|\nabla \eta^{t}(s)\right\|_{2}^{2} d s+C_{M f}\|\nabla w(t)\|_{2}^{2} .
\end{aligned}
$$

A desigualdade (4.28) segue fazendo $C_{1}>\max \left\{C_{\nu}, \frac{1}{2 \gamma_{0}}, C_{M f}\right\}$.

Etapa 4 Existe uma constante positiva $C_{2}$ tal que

$$
\begin{aligned}
\phi^{\prime}(t) \leq & -\frac{\nu}{2}\left\|w_{t}(t)\right\|_{2}^{2}-\frac{\nu}{2}\left\|\nabla w_{t}(t)\right\|_{2}^{2}+\frac{1}{8}\|\Delta w(t)\|_{2}^{2}+C_{2}\|\tau(t)\|_{2}^{2}+\omega C_{2}\|\nabla \tau(t)\|_{2}^{2} \\
& -C_{2} \int_{0}^{\infty} \mu^{\prime}(s)\left\|\nabla \xi^{t}(s)\right\|_{2}^{2} d s+C_{2}\|\nabla w(t)\|_{2}^{2} .
\end{aligned}
$$

Com efeito, derivando $\phi(t)$, temos

$$
\begin{aligned}
\phi^{\prime}(t)=\int_{\Omega} w_{t}(t) \tau_{t}(t) d x-\int_{\Omega} w_{t}(t) \Delta^{-1} \tau(t) d x & +\int_{\Omega} w_{t t}(t) \tau(t) d x \\
& -\int_{\Omega} w_{t t}(t) \Delta^{-1} \tau(t) d x .
\end{aligned}
$$

A partir da equação (4.14), e utilizando a desigualdade de Young para $\delta>0$, obtemos 


$$
\begin{aligned}
\int_{\Omega} w_{t}(t) \tau_{t}(t) d x & =\int_{\Omega} w_{t}(t)\left[\omega \Delta \tau(t)+\int_{0}^{\infty} \mu(s) \Delta \xi^{t}(s) d s+\nu \Delta w_{t}(t)\right] d x \\
& \leq \omega\left\|\nabla w_{t}(t)\right\|_{2}\|\nabla \tau(t)\|_{2}+\int_{0}^{\infty} \mu(s)\left\|\nabla w_{t}(t)\right\|_{2}\left\|\nabla \xi^{t}(s)\right\|_{2} d s-\nu\left\|\nabla w_{t}(t)\right\|_{2}^{2} \\
& \leq \delta\left\|\nabla w_{t}(t)\right\|_{2}^{2}+\omega C_{\delta \omega}\|\nabla \tau(t)\|_{2}^{2}+\delta k_{0}\left\|\nabla w_{t}(t)\right\|_{2}^{2}+C_{\delta}\left\|\xi^{t}\right\|_{\mu, 1}^{2} .
\end{aligned}
$$

Seja $0<\delta=\min \left\{\frac{\nu}{4 k_{0}}, \frac{\nu}{4}\right\}$ e $C>\max \left\{C_{\delta}, C_{\delta \omega}\right\}$, usando a observação 4.3 , concluimos que

$$
\int_{\Omega} w_{t}(t) \tau_{t}(t) d x \leq-\frac{\nu}{2}\left\|\nabla w_{t}(t)\right\|_{2}^{2}+\omega C\|\nabla \tau(t)\|_{2}^{2}-C \int_{0}^{\infty} \mu^{\prime}(s)\left\|\nabla \xi^{t}(s)\right\|_{2}^{2} d s .
$$

De maneira análoga e aumentando $C>0$ se necessário, temos

$$
\begin{aligned}
-\int_{\Omega} w_{t}(t) \Delta^{-1} \tau_{t}(t) d x & =-\int_{\Omega} w_{t}(t)\left[\omega \tau(t)+\int_{0}^{\infty} \mu(s) \xi^{t}(s) d s+\nu w_{t}\right] d x \\
& \leq-\frac{\nu}{2}\left\|w_{t}(t)\right\|_{2}^{2}+\omega C\|\nabla \tau(t)\|_{2}^{2}-C \int_{0}^{\infty} \mu^{\prime}(s)\left\|\nabla \xi^{t}(s)\right\|_{2}^{2} d s .
\end{aligned}
$$

Por outro lado, temos

$$
\begin{aligned}
-\int_{\Omega} w_{t t}(t) \Delta^{-1} \tau(t) d x= & -\int_{\Omega}\left[\Delta^{2} w(t)+M\left(\|\nabla v(t)\|_{2}^{2}\right) \Delta v(t)-M\left(\|\nabla u(t)\|_{2}^{2}\right) \Delta u(t)\right. \\
& \left.+\Delta w_{t t}(t)+f(v(t))-f(u(t))-\Delta \tau(t)\right] \Delta^{-1} \tau(t) d x \\
= & \nu\|\tau(t)\|_{2}^{2}+\int_{\Omega} \Delta w(t) \tau(t) d x-\int_{\Omega} w_{t t}(t) \tau(t) d x \\
& -\int_{\Omega}\left(M\left(\|\nabla v(t)\|_{2}^{2}\right) \Delta v(t)-M\left(\|\nabla u(t)\|_{2}^{2}\right) \Delta u(t)\right) \Delta^{-1} \tau(t) d x \\
& -\int_{\Omega}(f(v(t))-f(u(t))) \Delta^{-1} \tau(t) d x .
\end{aligned}
$$

De maneira análoga a feita na primeira etapa, podemos provar que

$$
\begin{aligned}
& \int_{\Omega}\left(M\left(\|\nabla v(t)\|_{2}^{2}\right) \Delta v(t)-M\left(\|\nabla u(t)\|_{2}^{2}\right) \Delta u(t)\right) \Delta^{-1} \tau(t) d x \leq C_{M \lambda_{1}}\|\nabla w(t)\|_{2}\|\tau(t)\|_{2} \\
& \leq C_{M \lambda_{1}}\|\nabla w(t)\|_{2}^{2}+C_{M \lambda_{1}}\|\tau(t)\|_{2}^{2}
\end{aligned}
$$


$\int_{\Omega}(f(v(t))-f(u(t))) \Delta^{-1} \tau(t) d x \leq C_{f \lambda_{1}}\|\nabla w(t)\|_{2}\|\tau(t)\|_{2} \leq C_{f \lambda_{1}}\|\nabla w(t)\|_{2}^{2}+C_{f \lambda_{1}}\|\tau(t)\|_{2}^{2}$.

Além disso, segue da desigualdade de Young que

$$
\int_{\Omega} \Delta w(t) \tau(t) d x \leq \frac{1}{8}\|\Delta w(t)\|_{2}^{2}+2\|\tau(t)\|_{2}^{2}
$$

Combinando as desigualdades acima, resulta

$$
\int_{\Omega} w_{t t}(t)\left[\tau(t)-\Delta^{-1} \tau(t)\right] d x \leq \frac{1}{8}\|\Delta w(t)\|_{2}^{2}+C_{M f \lambda_{1} \nu}\|\tau(t)\|_{2}^{2}+C_{M f \lambda_{1}}\|\nabla w(t)\|_{2}^{2}
$$

Portanto, a desigualdade (4.29) segue de (4.30), (4.31), (4.32) e (4.33), com $C_{2}=\max \left\{2 C, C_{M f \lambda_{1} \nu}, C_{M f \lambda_{1}}\right\}$

Etapa 5 Dado $\delta_{2}>0$, existe uma constante $C_{3}>0$ tal que

$$
\chi^{\prime}(t) \leq-\frac{k_{0}}{2}\|\tau(t)\|_{2}^{2}+\delta_{2}\left\|\nabla w_{t}(t)\right\|_{2}^{2}+\omega C_{3}\|\nabla \tau(t)\|_{2}^{2}-C_{3} \int_{0}^{\infty} \mu^{\prime}(s)\left\|\nabla \xi^{t}(s)\right\|_{2}^{2} d s
$$

De fato, notamos primeiramente que

$$
\chi^{\prime}(t)=-\int_{0}^{\infty} \mu(s)\left(\int_{\Omega} \xi^{t}(s) \tau_{t}(t) d x\right) d s-\int_{0}^{\infty} \mu(s)\left(\int_{\Omega} \tau(t) \xi_{t}^{t}(s) d x\right) d s .
$$

Utilizando a equação (4.14), observamos que

$$
\begin{aligned}
-\int_{0}^{\infty} \mu(s)\left(\int_{\Omega} \xi^{t}(s) \tau_{t}(t) d x\right) d s= & -\int_{0}^{\infty} \mu(s)\left(\omega \Delta \tau(t) \xi^{t}(s)\right) d s \\
& -\int_{0}^{\infty} \mu(s)\left(\int_{\Omega} \nu \Delta w_{t}(t) \xi^{t}(s)\right) d s \\
& -\int_{0}^{\infty} \mu(s)\left(\int_{\Omega}\left(\int_{0}^{\infty} \mu(s) \Delta \xi^{t}(s) d s\right) \xi^{t}(s) d x\right) d s .
\end{aligned}
$$

Por outro lado,

$$
-\int_{0}^{\infty} \mu(s)\left(\omega \Delta \tau(t) \eta^{t}(s)\right) d s \leq \omega k_{0}\|\nabla \tau(t)\|_{2}^{2}-C_{\omega} \int_{0}^{\infty} \mu^{\prime}(s)\left\|\nabla \xi^{t}(s)\right\|_{2}^{2} d s
$$


e

$$
\begin{aligned}
-\int_{\Omega}\left(\int_{0}^{\infty} \mu(s) \Delta \xi^{t}(s) d s\right) & \left(\int_{0}^{\infty} \mu(s) \xi^{t}(s) d s\right) d x \\
& =\int_{\Omega}\left(\int_{0}^{\infty} \mu(s) \nabla \xi^{t}(s) d s\right)\left(\int_{0}^{\infty} \mu(s) \nabla \xi^{t}(s) d s\right) d x \\
& =\int_{\Omega}\left|\int_{0}^{\infty} \mu(s) \nabla \xi^{t}(s) d s\right|^{2} d x \\
& =\int_{\Omega}\left|\int_{0}^{\infty} \mu(s)^{1 / 2} \mu(s)^{1 / 2} \nabla \xi^{t}(s) d s\right|^{2} d x \\
& \leq \int_{\Omega}\left(\int_{0}^{\infty} \mu(s) d s\right)\left(\int_{0}^{\infty} \mu(s)\left|\nabla \xi^{t}(s)\right|^{2} d s\right) d x \\
& \leq-\frac{k_{0}}{\gamma_{0}} \int_{0}^{\infty} \mu(s)\left\|\nabla \xi^{t}(s)\right\|_{2}^{2} d s .
\end{aligned}
$$

Agora, dado $\delta_{2}>, 0$ existe uma constante $C_{\delta_{2} \nu \gamma_{0}}>0$ tal que

$$
-\int_{0}^{\infty} \mu(s)\left(\omega \Delta w_{t}(t) \xi^{t}(s)\right) d s \leq \delta_{2}\left\|\nabla w_{t}(t)\right\|_{2}^{2}-C_{\delta_{2} \nu \gamma_{0}} \int_{0}^{\infty} \mu^{\prime}(s)\left\|\nabla \xi^{t}(s)\right\|_{2}^{2} d s .
$$

Para o outro termo do lado direito, notamos que, a partir da equação (4.15), temos

$$
\begin{aligned}
& -\int_{0}^{\infty} \mu(s)\left(\int_{\Omega} \tau(t) \xi_{t}^{t}(s) d x\right) d s=+\int_{0}^{\infty} \mu(s)\left(\int_{\Omega} \tau(t) \xi_{s}^{t}(s) d x\right) d s-k_{0}\|\tau(t)\|_{2}^{2} \\
= & -\int_{0}^{\infty} \mu^{\prime}(s)\left(\int_{\Omega} \tau(t) \xi_{s}^{t}(s) d x\right) d s-k_{0}\|\tau(t)\|_{2}^{2} \leq \frac{k_{0}}{2}\|\tau(t)\|_{2}^{2}-\frac{k_{1} C_{s}}{2 k_{0}} \int_{0}^{\infty} \mu^{\prime}(s)\left\|\nabla \xi^{t}(s)\right\|_{2}^{2} d s \\
& -k_{0}\|\tau(t)\|_{2}^{2}=-C_{k_{0} \gamma_{0}}\left\|_{2}^{2}-C_{k_{0}, k_{1}} \int_{0}^{\infty} \mu^{\prime}(s)\right\| \nabla \xi^{t}(s) \|_{2}^{2} d s .
\end{aligned}
$$

A estimativa para $\chi^{\prime}(t)$ resulta da combinação das desigualdades acima e fazendo $C_{3}=\max \left\{C_{k_{0}}, C_{\omega}+C_{k_{0} \gamma_{0}}+C_{\delta_{2} \nu \gamma_{0}}+C_{k_{0} k_{1}}\right\}$

Etapa 6 Existe $\epsilon>0$, suficientemente pequeno, e uma constante $C_{B}>0$, dependendo do subconjunto limitado $B$, tais que

$$
\mathcal{J}^{\prime}(t) \leq-\epsilon F(t)+C_{B}\|\nabla w(t)\|_{2}^{2}
$$

De fato, escolhemos $\epsilon_{1}>0$ de modo que $\epsilon_{1}<\frac{\nu}{6}$. Então, 


$$
\begin{array}{r}
\epsilon_{1} \psi^{\prime}(t)+\phi^{\prime}(t) \leq-\epsilon_{1} F(t)-\frac{\nu}{4}\left\|\nabla w_{t}(t)\right\|_{2}^{2}+\left(C_{1}+C_{2}\right)\|\tau(t)\|_{2}^{2} \\
+\left(C_{1}+C_{2}\right)\|\nabla w(t)\|_{2}^{2}+\omega C_{2}\|\nabla \tau(t)\|_{2}^{2}-\left(C_{1}+C_{2}\right) \int_{0}^{\infty} \mu^{\prime}(s)\left\|\nabla \xi^{t}(s)\right\|_{2}^{2} d s .
\end{array}
$$

Sejam $\delta_{2}, \epsilon_{2}>0$, tais que

$$
\epsilon_{2}\left(C_{1}+C_{2}\right)<\frac{k_{0}}{2} \quad \text { e } \quad \delta_{2}<\frac{\nu}{8}
$$

Assim,

$$
\begin{aligned}
\epsilon_{2}\left(\epsilon_{1} \psi^{\prime}(t)+\right. & \left.\phi^{\prime}(t)\right)+\chi^{\prime}(t) \leq-\epsilon_{1} \epsilon_{2} F(t)-\frac{\nu}{8}\left\|\nabla w_{t}(t)\right\|_{2}^{2}+\left(C_{1}+C_{2}\right)\|\nabla w(t)\|_{2}^{2} \\
& +\omega\left(C_{2}+C_{3}\right)\|\nabla \tau(t)\|_{2}^{2}-\left(C_{1}+C_{2}+C_{3}\right) \int_{0}^{\infty} \mu^{\prime}(s)\left\|\nabla \xi^{t}(s)\right\|_{2}^{2} d s .
\end{aligned}
$$

Escolhendo $N>\max \left\{2 \beta_{0}, 2\left(C_{1}+C_{2}+C_{3}\right)\right\}$ e $0<\delta_{0}<\frac{\nu}{16 N}$, concluímos que

$$
\mathcal{J}^{\prime}(t) \leq-\epsilon_{1} \epsilon_{2} F(t)+\left(N C_{\delta_{0}}+C_{5}+C_{6}\right)\|\nabla w(t)\|_{2}^{2}
$$

Assim, fazendo $C_{B}=\left(N C_{\delta_{0}}+C_{5}+C_{6}\right)$ obtemos (4.36).

Conclusão A partir das desigualdades (4.27) e (4.36), obtemos

$$
\mathcal{J}^{\prime}(t) \leq-\frac{\epsilon}{\beta_{2}} \mathcal{J}(t)+C_{B}\|\nabla w(t)\|_{2}^{2}
$$

Seja $\sigma=\frac{\epsilon}{\beta_{2}}$. Logo,

$$
\mathcal{J}(t) \leq e^{-\sigma t} \mathcal{J}(0)+C_{B} \int_{0}^{t} e^{-\sigma(t-s)}\|\nabla w(s)\|_{2}^{2} d s .
$$

Finalmente, com $C=\frac{\beta_{2}}{\beta_{1}}$ e $K_{B}=\frac{C_{B}}{\beta_{1}}$, observamos que

$$
F(t) \leq C e^{-\sigma t} F(0)+K_{B} \int_{0}^{t} e^{-\sigma(t-s)}\|\nabla w(s)\|_{2}^{2} d s .
$$

Nossa demonstração segue dessa última desigualdade. 


\subsubsection{Quase-estabilidade}

Nesta seção, mostraremos que o sistema dinâmico produzido pelo problema (3.6)-(3.8) é quase-estável.

Lema 4.4. (Quase-estável) Suponhamos que as condições do Teorema 4.1 sejam válidas. Então o sistema dinâmico associado ao problema (3.6)-(3.8) é quase-estável para todo conjunto $\mathcal{B} \subset \mathcal{H}_{0}$ limitado e positivamente invariante.

Demonstração. Como já mencionado na observação 4.1, o problema (3.6)-(3.8) produz o sistema dinâmico $\left(\mathcal{H}_{0}, S(t)\right)$, o qual satisfaz as condições $(2.8)$ e $(2.9)$, em que

$$
S(t)\left(u_{0}, u_{1}, \theta_{0}, \eta_{0}\right)=\left(u(t), u_{t}(t), \theta(t), \eta^{t}\right),
$$

com $\left(u_{0}, u_{1}, \theta_{0}, \eta_{0}\right) \in \mathcal{H}_{0}$. Dado um subconjunto limitado e positivamente invariante $\mathcal{B}$, de $\mathcal{H}_{0}$, sejam $z_{0}^{1}=\left(u_{0}^{1}, u_{1}^{1}, \theta_{0}^{1}, \eta_{0}^{1}\right)$ e $z_{0}^{2}=\left(u_{0}^{2}, u_{1}^{2}, \theta_{0}^{2}, \eta_{0}^{2}\right)$ tais que $z_{0}^{1}, z_{0}^{2} \in \mathcal{B}$. Se $z^{1}(t)$ e $z^{2}(t)$ são as respectivas soluções por $z_{0}^{1}$ e $z_{0}^{2}$, então $z^{1}(t)=S(t) z_{0}^{1}$ e $z^{2}(t)=S(t) z_{0}^{2}$. Pelo Teorema 3.1 , temos

$$
\left\|S(t) z_{0}^{1}-S(t) z_{0}^{2}\right\|_{\mathcal{H}_{0}}^{2} \leq a(t)\left\|z_{0}^{1}-z_{0}^{2}\right\|_{\mathcal{H}_{0}}^{2}
$$

em que $a(t)=e^{\varrho t}$. É imediato que $a(t)$ é uma função positiva localmente limitada no intervalo $[0, \infty)$. Por outro lado, decorre da desigualdade de estabilização que

$$
\left\|S(t) z_{0}^{1}-S(t) z_{0}^{2}\right\|_{\mathcal{H}_{0}}^{2} \leq C e^{-\sigma t}\left\|z_{0}^{1}-z_{0}^{2}\right\|_{\mathcal{H}_{0}}^{2}+K_{B} \int_{0}^{t} e^{-\sigma(t-s)}\left\|\nabla u^{1}(s)-\nabla u^{2}(s)\right\|_{2}^{2} d s
$$

para todo $t \geq 0$. Logo,

$$
\left\|S(t) z^{1}-S(t) z^{2}\right\|_{\mathcal{H}_{0}}^{2} \leq C e^{-\sigma t}\left\|z^{1}-z^{2}\right\|_{\mathcal{H}_{0}}^{2}+\frac{K_{B}}{\sigma} \sup _{0 \leq s \leq t}\left\|\nabla u^{1}(s)-\nabla u^{2}(s)\right\|_{2}^{2}
$$

Definimos

$$
\begin{aligned}
& n_{V_{2}}: V_{2} \longrightarrow \mathbb{R}^{+}, \\
& n_{V_{2}}(u(t))=\|\nabla u(t)\|_{2} .
\end{aligned}
$$


Sejam $b(t)=C e^{-\sigma t}$ e $c(t)=\frac{K_{B}}{\sigma}$. É claro que $c(t)$ é localmente limitada em $[0, \infty)$ e $b(t)$ é uma função de $L^{\infty}\left(\mathbb{R}^{+}\right)$, satisfazendo $\lim _{t \rightarrow \infty} b(t)=0$. Assim, reescrevemos a desigualdade de estabilização como segue

$$
\left\|S(t) z^{1}-S(t) z^{2}\right\|_{\mathcal{H}_{0}}^{2} \leq b(t)\left\|z^{1}-z^{2}\right\|_{\mathcal{H}_{0}}^{2}+c(t) \sup _{0 \leq s \leq t}\left[n_{V_{2}}\left(u^{1}(s)-u^{2}(s)\right)\right]^{2} .
$$

Resta checar que a seminorma $n_{V_{2}}$ é pré-compacta em $V_{2}$. Entretanto, isso é consequência do fato que a inclusão $V_{2} \hookrightarrow V_{1}$ é compacta. Portanto, segue da definição2.17 que $\left(\mathcal{H}_{0}, S(t)\right)$ é quase-estável sobre $\mathcal{B}$.

\subsection{Demonstração do teorema 4.1}

Finalmente, mostraremos, através dos resultados estabelecidos nas seções anteriores, que existe um atrator global $\mathcal{A}$ com dimensão fractal finita.

Demonstração. Pelo Lema 4.4, o sistema dinâmico gerado pelo problema (3.6)-(3.9) é quase-estável sobre conjuntos limitados e positivamente invariantes de $\left(\mathcal{H}_{0}, S(t)\right)$. Então, segue da Proposição 2.1 que nosso sistema dinâmico é assintoticamente suave. Além disso, aplicando o Lema 4.1, temos que o sistema dinâmico $\left(\mathcal{H}_{0}, S(t)\right)$ é gradiente com funcional de Lyapunov $\Phi(x)=E(t)$. Claro que $\Phi$ é limitado sobre limitados de $\mathcal{H}_{0}$. Dado $R>0$, definimos o conjunto

$$
\Phi_{R}=\left\{U \in \mathcal{H}_{0} ; \Phi(U)<R\right\}
$$

Segue do Lema 3.4 o conjunto $\Phi_{R}$ é limitado, pois para $U \in \Phi_{R}$, a solução $U(t)$ do problema (3.25) associada a $U$ satisfaz

$$
\|U(t)\|_{\mathcal{H}_{0}}^{2} \leq \frac{1}{\delta_{0}}\left(\Phi(U(t))+C_{M f h}\right) \leq \frac{R+C_{M f h}}{\delta_{0}}, \forall t \geq 0 .
$$

Em partícular, $U$ satisfaz essa desigualdade. O que mostra que $\Phi_{R}$ é limitado. No Lema 4.2, mostramos que o conjunto $\mathcal{N}$ das soluções estacionárias é limitado. Assim, estamos nas hipóteses do Corolário 2.2, logo o sistema dinâmico $\left(\mathcal{H}_{0}, S(t)\right)$ possui um 
atrator global $A=\mathcal{W}^{u}(\mathcal{N})$. Resta mostrar que $\mathcal{A}$ tem dimensão finita. Como $\mathcal{A}$ é invariante, aplicando o Lema 4.4, concluimos que $\left(\mathcal{H}_{0}, S(t)\right)$ é quase-estável sobre $\mathcal{A}$. Logo, pelo Teorema 2.18, nosso atrator global tem dimensão fractal finita. 



\section{Capítulo 5}

\section{Atratores exponenciais}

Neste capítulo estudamos a existência de atratores exponenciais generalizados. Seguiremos o método apresentado em Chueshov \& Lasiecka [12, 13], que é baseado no conceito de quase-estabilidade. Como referência clássica citamos o trabalho de Eden et al [14] que usa a chamada propriedade de compressão (squeezing property).

\subsection{Atratores exponenciais generalizados}

Lema 5.1. Nas condições do Teorema 4.1, o sistema dinâmico $\left(\mathcal{H}_{0}, S(t)\right)$, dado pelo Teorema 3.1, é dissipativo.

Demonstração. Provamos no Lema 4.1 que o sistema dinâmico $\left(\mathcal{H}_{0}, S(t)\right)$ é gradiente com funcional de Lyapunov $\Phi=E$. Mostraremos que $\mathcal{B}=\left\{U \in \mathcal{H}_{0} ; \Phi(U) \leq R\right\}$ é um conjunto absorvente, limitado e positivamente invariante, se $R>0$ é suficientemente grande.

De fato, o conjunto $\Phi_{R}=\left\{U \in \mathcal{H}_{0} ; \Phi(U) \leq R\right\}$ é positivamente invariante para todo $R>0$, pois dado $U_{0} \in \Phi_{R}$, existe uma única solução $U \in C\left([0, \infty), \mathcal{H}_{0}\right)$ tal que $U(t)=S(t) U$. Por outro lado, como $\Phi$ é um funcional de Lyapunov estrito, logo $\Phi(U(t)) \leq \Phi\left(U_{0}\right)$, para todo $t \geq 0$, isto é, $\Phi(U(t)) \leq \Phi\left(U_{0}\right) \leq R$, para todo $t \geq 0$ e $R>0$. Portanto, $S(t) \Phi_{R} \subset \Phi_{R}$, para todo $t \geq 0$. Na demonstração do Teorema 4.1 mostramos que para cada $R>0$, o conjunto $\Phi_{R}$ é limitado.

Agora, como o Atrator global $\mathcal{A}$ é compacto, existe um $R_{0}>0$ tal que 


$$
\mathcal{A} \subset B\left(0, R_{0}\right) \subset\left\{U \in \mathcal{H}_{0} ; \Phi(U) \leq R\right\},
$$

para $R$ suficientemente grande. Seja $R_{0}$ tal que inclusão (5.1) ocorra e tome $\mathcal{B}=\Phi_{R_{0}}$. Finalmente, temos que $\mathcal{B}$ é um absorvente, pois dado $B \subset \mathcal{H}_{0}$, temos

$$
\lim _{t \rightarrow \infty} \operatorname{dist}_{\mathcal{H}_{0}}(S(t) B, \mathcal{A})=0,
$$

isto é, existe $T_{B}>0$ tal que $S(t) B \subset \mathcal{B}$, para todo $t \geq T_{B}$.

Teorema 5.1. Suponha que as hipóteses do Teorema 4.1 sejam válidas. Então para cada $\delta \in(0,1]$, o sistema dinâmico associado ao problema (3.6)-(3.8) possui um atrator exponencial generalizado, com dimensão fractal finita no espaço estendido

$$
\mathcal{H}_{-\delta}=V_{2-\delta} \times V_{1-\delta} \times V_{-\delta} \times L_{\mu}^{2}\left(\mathbb{R}^{+} ; V_{1-\delta}\right),
$$

em que os espaços de Sobolev $V_{s-\delta}$ serão definidos na demonstração desse teorema.

Demonstração. Para mostrar a existência do atrator exponencial generalizado, aplicaremos o Teorema 2.19. Vamos primeiro mostrar que o resultado vale para $\delta=1$. Em seguida utilizamos a interpolação $\left[\mathcal{H}_{0}, \mathcal{H}_{-1}\right]_{\delta}, \operatorname{com} \delta \in(0,1]$.

Seja $\mathcal{B}$ o absorvente limitado e positivamente invariante do sistema dinâmico $\left(\mathcal{H}_{0}, S(t)\right)$, dado pelo Lema 5.1. Provamos anteriormente que $\left(\mathcal{H}_{0}, S(t)\right)$ é quase estável sobre qualquer subconjunto limitado positivamente invariante de $\mathcal{H}_{0}$, o que, em particular, vale para $\mathcal{B}$.

Segundo o Teorema 2.19, é preciso também mostrar que $t \mapsto S(t) y$ é Hölder contínua em $\mathcal{H}_{-\delta}$. Para esse fim vamos considerar potências fracionárias de operadores. Definimos o operador $A$ pela terna $\left\{V_{1}, V_{0},(\cdot, \cdot)\right\}$ e

$$
\langle A u, v\rangle=(\nabla u, \nabla v), \quad \forall u, v \in V_{1},
$$

em que $\langle\cdot, \cdot\rangle$ é a dualidade entre $V_{-1}$ e $V_{1}$. Nesse caso, $D(A)=H^{2}(\Omega) \cap H_{0}^{1}(\Omega)=V_{2}$ e $A u=-\Delta u, u \in V_{2}$. Pela observação 2.2 do capítulo 2 subseção 2.2 , sabemos que o operador $A$ é densamente definido em $V_{0}$, auto-adjunto e definido positivo em $V_{1}$. 
Portanto, podemos definir as potências de $A$, ou seja, $A^{s / 2}, s \in \mathbb{R}$, com domínio $D\left(A^{s / 2}\right)$ munido com o produto interno e norma

$$
(u, v)_{D\left(A^{s / 2}\right)}=\left(A^{s / 2} u, A^{s / 2} v\right) \text { e }\|u\|_{D\left(A^{s / 2}\right)}=\left\|A^{s / 2} u\right\|_{2}, \forall u, v \in D\left(A^{s / 2}\right)
$$

respectivamente. Em particular, $D\left(A^{1 / 2}\right)=V_{1}$. Utilizando o Lema 2.6, se $s_{1} \geq s_{2}$, a imersão

$$
D\left(A^{s_{1}}\right) \hookrightarrow D\left(A^{s_{2}}\right)
$$

é contínua. No caso de $s_{1}>s_{2}$, a imersão 5.5 é compacta. Com essa notação em mãos, a norma de $\mathcal{H}_{-\delta}$ se escreve

$$
\|U(t)\|_{\mathcal{H}_{-\delta}}^{2}=\|u(t)\|_{V_{2-\delta}}^{2}+\left\|u_{t}(t)\right\|_{V_{1-\delta}}^{2}+\|\theta(t)\|_{V_{-\delta}}^{2}+\left\|\eta^{t}\right\|_{\mu, 1-\delta}^{2}
$$

em que $V_{s-\delta}=D\left(A^{s-\delta / 2}\right)$ e

$$
\left\|\eta^{t}\right\|_{\mu, 1-\delta}^{2}=\int_{0}^{\infty} \mu(s)\left\|\eta^{t}(s)\right\|_{V_{1-\delta}}^{2} d s .
$$

Seja $U(t)$ uma solução clássica do problema (3.25) com dado inicial $y=U_{0} \in \mathcal{B}$ definida no intervalo $[0, T]$. Como $\mathcal{B}$ é limitado, segue do Lema 3.4 que $U(t)$ é globalmente limitada. Então, como $\mathcal{L}$ é um $C_{0^{-}}$semigrupo de contrações, existe uma constante $C_{B T}>0$ tal que $\|\mathcal{L} U(t)\|_{\mathcal{H}_{0}} \leq\|U(t)\|_{\mathcal{H}_{0}} \leq C_{\mathcal{B} T}$. Além disso, $F$ é localmente Lipschitz contínua em $\mathcal{H}_{0}$, o que implica que existe uma constante $\widetilde{C}_{\mathcal{B} T}>0$ tal que $\|F(U(t))\|_{\mathcal{H}_{0}} \leq \widetilde{C}_{\mathcal{B} T}>0$. Logo, do problema (3.25), observamos que

$$
\begin{aligned}
\|\dot{U}(t)\|_{\mathcal{H}_{-1}} & \leq\|\mathcal{L} U(t)\|_{\mathcal{H}_{-1}}+\|F(U(t))\|_{\mathcal{H}_{-1}} \\
& \leq\|\mathcal{L} U(t)\|_{\mathcal{H}_{0}}+\|F(U(t))\|_{\mathcal{H}_{0}} \\
& \leq \mathcal{C}_{\mathcal{B} T}
\end{aligned}
$$

na qual $\mathcal{C}_{\mathcal{B} T}$ é uma constante positiva. Logo, se $t_{2}, t_{1} \in[0, T]$ e supomos, sem perda de generalidade que $t_{2}>t_{1}$, teremos 


$$
\left\|S\left(t_{2}\right) y-S\left(t_{1}\right) y\right\| \leq \int_{t_{1}}^{t_{2}}\|\dot{U}(s)\|_{\mathcal{H}_{-1}} d s \leq C_{\mathcal{B} T}\left|t_{2}-t_{1}\right| .
$$

Destacamos que a desigualdade acima prova a existência de um atrator exponencial quando $\delta=1$. Para o caso $\delta \in(0,1)$, afirmamos que existe uma constante $C_{\mathcal{B} T}>0$ tal que

$$
\left\|S\left(t_{1}\right) y-S\left(t_{2}\right) y\right\|_{\mathcal{H}_{-\delta}} \leq C_{\mathcal{B} T}\left\|S\left(t_{1}\right) y-S\left(t_{2}\right) y\right\|_{\mathcal{H}_{-1}}^{\delta} .
$$

De fato, consideramos as seguintes imersões compactas

$$
V_{0} \hookrightarrow V_{-\delta} \hookrightarrow V_{-1}
$$

Então temos a seguinte desigualdade de interpolação

$$
\|u\|_{V_{-\delta}} \leq\|u\|_{2}^{1-\delta}\|u\|_{V_{-1}}^{\delta}, \forall u \in V_{0} .
$$

Aplicaremos essa desigualdade em (5.6). Então,

$$
\begin{aligned}
\|U(t)\|_{\mathcal{H}_{-\delta}}^{2} \leq & \|u(t)\|_{V_{2}}^{2(1-\delta)}\|u(t)\|_{V_{1}}^{2 \delta}+\left\|u_{t}(t)\right\|_{V_{1}}^{2(1-\delta)}\left\|u_{t}(t)\right\|_{2}^{2 \delta}+\|\theta(t)\|_{2}^{2(1-\delta)}\|\theta(t)\|_{V_{-1}}^{2 \delta} \\
& +\int_{0}^{\infty} \mu(s)\left\|\eta^{t}(s)\right\|_{V_{1}}^{2(1-\delta)}\left\|\eta^{t}(s)\right\|_{2}^{2 \delta} d s .
\end{aligned}
$$

Pela Desigualdade de Hölder, com

$$
p=\frac{1}{1-\delta} \text { e } q=\frac{1}{\delta}
$$

temos

$$
\|U(t)\|_{\mathcal{H}_{0}} \leq\|U(t)\|_{\mathcal{H}_{0}}^{1-\delta}\|U(t)\|_{\mathcal{H}_{-1}}^{\delta},
$$

o que nos leva a

$$
\|U(t)\|_{\mathcal{H}_{0}} \leq \mathcal{C}_{\mathcal{B}, T}\|U(t)\|_{\mathcal{H}_{-1}}^{\delta}
$$

Consequentemente, a desigualdade (5.9) segue de (5.14). Portanto, existe uma constante $\mathcal{C}_{\mathcal{B} T}>0$ de forma que 
$\left\|S\left(t_{1}\right) y-S\left(t_{2}\right) y\right\|_{\mathcal{H}_{-\delta}} \leq \mathcal{C}_{\mathcal{B} T}\left|t_{1}-t_{2}\right|^{\delta}$. 


\section{Referências Bibliográficas}

[1] R. A. Adams, Sobolev Spaces. Academic Press, New York, 1975.

[2] A. V. Babin \& M. I. Vishiki, Attractors of Evolution Equations, Studies in Mathematics and its Applications 25, North-Holland, Amsterdam, 1992.

[3] Alisson R. A. Barbosa \& T. F. Ma, Long-time dynamics of an extensible plate equation with thermal memory, Preprint, ICMC-USP (2013).

[4] H. M. Berger, A new approach to the analysis of large deflections of plates, Journal of Applied Mechanics 22 (1955) 465-472.

[5] H. Brezis, Analyse Fonctionnelle: Theórie et Applications. Masson, Paris, 1983.

[6] A.N.Carvalho, J. Langa \& J.C. Robinson,Attractors for Infinite-Dimensional NonAutonomous Dynamical Systems, Applied Mathematical Sciences 182, Springer, New York, 2013.

[7] C. Cattaneo, Atti Sem. Mat. Fis. Moderna 3 (1948) 83-101; C.R. Acad. Sc. Paris 247 (1958) 431-433.

[8] C. Cattaneo, Atti Sem. Mat. Fis. Moderna 3 (1948) 83-101; C.R. Acad. Sc. Paris 247 (1958) 431-433.

[9] M. M. Cavalcanti \& V. N. Domingos Cavalcanti, J. A. Soriano, Global existence and asymptotic stability for the nonlinear and generalized damped extensible plate equation, Comm. Contemporary Math. 6 (2004) 705-731. 
[10] I. Chueshov \& I. Lasiecka, Attractors for second order evolution equations with a nonlinear damping, J. Dyn. Diff. Eq., 16 (2004), 469-512.

[11] I. Chueshov \& I. Lasiecka, Long Time Dynamics of Von Karman Evolutions with Thermal Effects, Bol. Soc. Parana. Mat. (3) 25 (2007), no. 1-2, 37-54.

[12] I. Chueshov \& I. Lasiecka, Long-Time Behavior of Second Order Evolution Equations with Nonlinear Damping, Mem. Amer. Math. Soc. 195, no. 912, Providence, 2008.

[13] I. Chueshov \& I. Lasiecka, Von Karman Evolution Equations Well-posedness and Long-Time Dynamics, New York, Springer, 2010.

[14] A. Eden, C. Foias, B. Nicolaenko \& R. Temam, Exponential attractors for dissipative evolution equations, Research in Applied Mathematics, vol. 37, John Wiley \& Sons, New York, 1994.

[15] H. D. Fernández Sare, R. Racke, On the stability of damped Timoshenko systems: Cattaneo versus Fourier law. Arch. Ration. Mech. Anal. 194 (2009) 221-251.

[16] G. B. Folland, Real analysis - Modern techniques and their applications, Second edition, Pure and Applied Mathematics, John Wiley \& Sons, Inc., New York, 1999.

[17] C. Giorgi, A. Marzocchi \& V. Pata, Asymptotic behavior of a semilinear problem in heat conduction with memory, NoDEA Nonlinear Diff. Eq. Appl. 5, (1998) 333-354.

[18] C. Giorgi \& V. Pata, Stability of abstract linear thermoelastic systems with memory, Math. Models Meth. Appl. Sci. (M3AS) 11 (2001) 627-644.

[19] C. Giorgi, M. Grasseli \& V. Pata, Well-posedness and longtime behavior of the phase-field model with memory in a history space setting, Quart. Appl. Math. 59 (2001) 701-736.

[20] C. Giorgi, V. Pata \& E. Vuk, On the extensible viscoelastic beam, Nonlinearity 21 (2008) 713-733. 
[21] C. Giorgi, M. G. Naso, V. Pata \& M. Potomkin, Global attractors for the extensible thermoelastic beam system, J. Differential Equations 246 (2009), 3496-3517.

[22] M. Grasselli \& V. Pata, Uniform attractors of nonautonomous dynamical systems with memory, Progr. Nonlinear Differential Equations Appl. 50 (2002) 155-178.

[23] M. Grasselli \& J. E. Muñoz Rivera \& V. Pata, On the energy decay of the linear thermoelastic plate with memory, J. Math. Anal. Appl. 309 (2005) 1-14.

[24] M. Grasselli \& M. Squassina, Exponential stability and singular limit for a linear thermoelastic plate with memory effects, Adv. Math. Sci. Appl. 16 (2006) 15-31.

[25] M. E. Gurtin \& A. C. Pipkin, A general theory of heat conduction with finite wave speeds. Arch. Rational Mech. Anal. 31 (1968) 113-126.

[26] J. K. Hale, Asymptotic behavior of dissipative systems, Mathematical Surveys and Monographs, 25. American Mathematical Society, Providence, RI, 1988.

[27] S. Kesavan, Topics in Functional Analysis and Applications. Copyright, Bangalore, India, 1989.

[28] E. Kreyszig, Introductory Functional Analysis with Applications. John Wiley e Sons, 1978.

[29] O. Ladyzhenskaya, Attractors for Semi-groups and Evolution Equations, Cambridge University Press, 1991.

[30] J.-L. Lions, Quelques Méthodes de Résolution des Problèmes aux Limites Non Linéaires, Dunod Gauthier-Villars, Paris 1969.

[31] J.-L. Lions \& E. Magenes, Non-Homogeneous Boundary Value Problems and Applications, Vol.I, Springer-Verlag, Berlin, 1972.

[32] T.F. Ma \& V. Narciso, Pelicer, Long-time behavior of a model of extensible beams with nonlinear boundary dissipations, J. Math. Anal. Appl. 396 (2012) 694-703. 
[33] A. Miranville, S. Zelik, Handbook of Differential Equations, Evolutionary Equations, Volume 4, Chapter 3, C.M. Dafermos and M. Pokorny, Editors, Elsevier, 2008 .

[34] V. Pata \& S. Zelik, Global and exponential attractors for 3-D wave equations with displacement dependent damping, Math. Meth. Appl. Sci. 29 (2006), no. 11, 12911306.

[35] A. Pazy, Semigroups of Linear Operators and Applications to Partial Differential Equations. Springer-Verlag, New York, 1983.

[36] M. Potomkin,Asymptotic behavior of thermoviscoelastic Berger plate, Commun. Pure Appl. Anal. 9 (2010) 161-192.

[37] J. Simon, Compact sets in the space $L^{p}(0, T ; B)$, Ann. Mat. Pura Appl. (4) 146 (1987), 65-96.

[38] B. Straughan, Heat Waves, Springer, New York, 2011.

[39] R. Temam, Infinite-Dimensional Dynamical Systems in Mechanics and Physics, Applied Mathematical Sciences 68, Springer-Verlag, New York, 1988.

[40] S. Woinowsky-Krieger, The effect of axial force on the vibration of hinged bars, Journal of Applied Mechanics 17 (1950) 35-36.

[41] H. Wu, Long-time behavior for a nonlinear plate equation with thermal memory, J. Math. Ana. Appl. 348 (2008) 650-670.

[42] K. Yosida, Functional analysis. Springer-Verlag, Berlim-Heildelberg, New York, 1966.

[43] S.Zheng. Nolinear parabolic equations and hiperbolic-parabolic coupled systems. Pitman series Monographs and Survey in Pure and Apllied Mathematics. Vol. 76.Logman,1995.

[44] Yang Zhijian, On an extensible beam equation with nonlinear damping and source terms, J. Differential Equations 254 (2013) 3903-3927. 